\title{
DEVELOPING LIGHT COLLECTION ENHANCEMENTS AND WIRE TENSIONING METHODS FOR LArTPC NEUTRINO DETECTORS
}

A dissertation SUbmitted to the University of Manchester FOR THE DEGREe of Master of ScIENCE By RESEARCH In the Faculty of Science And Engineering 


\section{Contents}

$\begin{array}{ll}\text { Abstract } & 10\end{array}$

$\begin{array}{ll}\text { Declaration } & 11\end{array}$

$\begin{array}{ll}\text { Copyright Statement } & 12\end{array}$

$\begin{array}{ll}\text { Acknowledgements } & 13\end{array}$

$\begin{array}{llr}1 & \text { Introduction } & 14\end{array}$

2 Neutrino Physics $\quad 17$

2.1 Neutrinos . . . . . . . . . . . . . . . . . . . . . . . . . . . 17

2.1.1 Neutrino interactions . . . . . . . . . . . . . . . . . . . . . 17

2.2 Neutrino oscillation . . . . . . . . . . . . . . . . . . . . . . . 18

2.3 Sterile neutrino . . . . . . . . . . . . . . . . . . . . 21

3 Short-Baseline Neutrino Program 23

3.1 Introduction . . . . . . . . . . . . . . . . . . 23

3.2 Sensitivity to sterile neutrinos . . . . . . . . . . . . . 25

3.3 Short Baseline Near Detector (SBND) . . . . . . . . . . . . . . 25

4 Liquid Argon Time Projection Chambers $\quad 28$

4.1 Introduction . . . . . . . . . . . . . . . . . . . . . . . . . 28

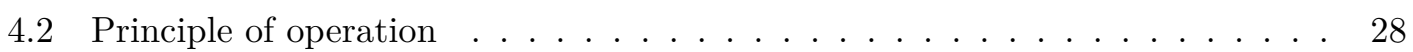

4.2.1 Charge signal collection and reconstruction . . . . . . . . . . . 28

$4.2 .2 \quad$ Scintillation light . . . . . . . . . . . . . . . . . . . . . 30

4.3 Energy reconstruction and particle identification $\ldots \ldots \ldots . \ldots . \ldots 31$

5 The SBND and LArIAT Detectors 33

5.1 The Short Baseline Near Detector (SBND) $\ldots \ldots \ldots$ 
5.1 .1 Light collection system . . . . . . . . . . . . . . . . . 37

5.2 Liquid Argon in A Test Beam (LArIAT) . . . . . . . . . . . . . . . 37

5.2 .1 Beam line . . . . . . . . . . . . . . . . . . . . 38

$5.2 .2 \quad$ Detector . . . . . . . . . . . . . . . . . . 38

5.2 .3 Light collection system . . . . . . . . . . . . . . . . . . . . 40

6 Principles of an Electrical Wire Tensioning Measurement for LArTPCs 42

6.1 Introduction . . . . . . . . . . . . . . . . . . . 42

6.2 Generic principle of tension measurements . . . . . . . . . . . . . . . 43

6.3 Laser technique . . . . . . . . . . . . . . . . . . . . . 45

6.4 Electric technique . . . . . . . . . . . . . . . . . . 46

6.5 Fit model and frequency extrapolation . . . . . . . . . . . . . . . . 48

7 Developing the Tension Measurement Method for SBND 50

7.1 Electronic circuits and wire frame set-ups . . . . . . . . . . . . . 50

7.1 .1 Wire-frame set-ups . . . . . . . . . . . . . . . . 50

7.1 .2 Wire soldering and tension preparation $\ldots \ldots \ldots \ldots 2$

7.1.3 SBND boards and limitations . . . . . . . . . . . . . . . . 54

7.1.4 Electronic circuits _. . . . . . . . . . . . . . . . . . . 54

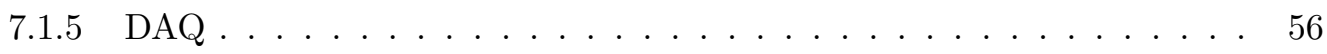

7.2 Evolution of the circuit and method $\ldots \ldots \ldots \ldots \ldots$

7.2 .1 Early measurements and noise reduction . . . . . . . . . . 58

7.2 .2 Upgrade of the circuit to use three wires . . . . . . . . . . . . 60

7.2 .3 Resolution . . . . . . . . . . . . . . . . . . 65

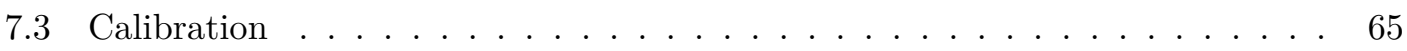

$7.3 .1 \quad$ Laser set-up . . . . . . . . . . . . . . . . . . . 66 66

7.3.2 Electric method calibration . . . . . . . . . . . . . . 69

7.4 Test at cryogenic temperatures $\ldots \ldots \ldots \ldots \ldots \ldots \ldots$

7.4 .1 Experimental set-up . . . . . . . . . . . . . . . . . 71

7.4 .2 Measurement procedure . . . . . . . . . . . . . . 72

$7.4 .3 \quad$ Expectations and analysis . . . . . . . . . . . . 73

7.5 SBND dedicated development . . . . . . . . . . . . . . 76

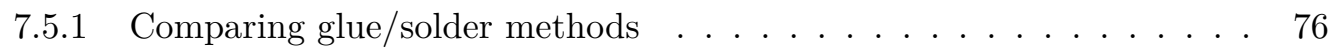

7.5 .2 Measurements with combs . . . . . . . . . . . . . . . . 79

7.5.3 Test of 32 -channel readout with test frame $\ldots \ldots \ldots 79$ 


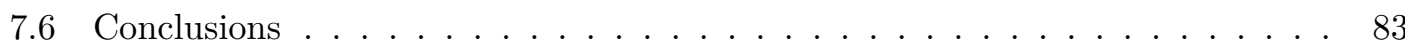

8 Developing Wavelength-Shifter Covered Reflector Foils for LArTPCs 84

8.1 Introduction . . . . . . . . . . . . . . . . . . . 84

8.2 Testing substrate foils $\ldots \ldots \ldots \ldots \ldots \ldots$

8.2.1 Material Test Stand tests . . . . . . . . . . . . . . . . . 86

8.2 .2 Testing foil behaviour at LAr temperatures $\ldots \ldots \ldots$. . . . . . 86

8.2 .3 Summary of foil tests . . . . . . . . . . . . . . . . . . . 92

8.3 Low Temperature Evaporation (LTE) _ . . . . . . . . . . . . . . . . . 92

8.4 Setting up the large-scale evaporator . . . . . . . . . . . . . . . . . . 93

8.4 .1 Installation . . . . . . . . . . . . . . . . . . . . . . . 93

8.4 .2 Evaporation procedure . . . . . . . . . . . . . . . . . 95

8.4 .3 First evaporation and dunking test . . . . . . . . . . . . . . . 97

8.4 .4 Evaporator calibration . . . . . . . . . . . . . . . . . . . . . 98

8.5 Foils and mesh cathode in LArIAT . . . . . . . . . . . . . . . . . . . 100

8.5.1 Mesh cathode . . . . . . . . . . . . . . . . . . 100

8.5.2 LArIAT run with evaporated reflector foils . . . . . . . . . . . . . 101

8.6 Conclusion . . . . . . . . . . . . . . . . . . . . . . 102

9 Beam Shape Studies for Delayed Ion Modelling 103

9.1 Introduction . . . . . . . . . . . . . . . . . . . . . . . . . . 103

9.2 LArIAT data runs . . . . . . . . . . . . . . . . . . . . . . . . 104

9.3 Simulation procedure . . . . . . . . . . . . . . . . . . . . 105

9.3.1 Beam simulation . . . . . . . . . . . . . . . . . . 105

$9.3 .2 \quad$ Cosmic ray simulation . . . . . . . . . . . . . . . . 106

9.3 .3 Comparison with data . . . . . . . . . . . . . . . . . 107

9.4 Conclusion . . . . . . . . . . . . . . . . . . . . . . . . . 108

$\begin{array}{ll}10 \text { Conclusions } & 109\end{array}$

$\begin{array}{ll}\text { Bibliography } & 111\end{array}$

Word count 20,597 


\section{List of Tables}

2.1 Feynman diagrams of neutrino interaction vertices. . . . . . . . . 18

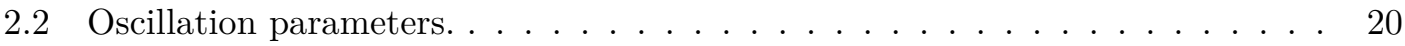

5.1 SBND TPC key design parameters. . . . . . . . . . . . . . . . . . 34

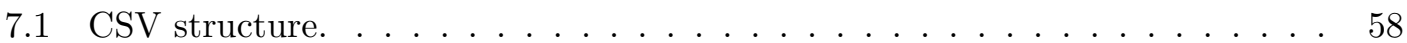

7.2 Lengths and corresponding nominal frequency for the wires measured. . . . . 68

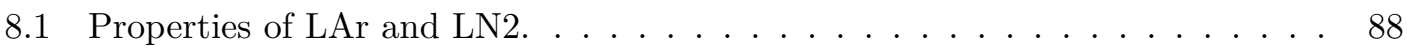

8.2 Summary of foil tests in liquid nitrogen. . . . . . . . . . . . . . . . 92 


\section{List of Figures}

2.1 Flavour composition of mass eigenstates for normal and inverted hierarchy. . 20

2.2 Electron-neutrino excess observed by the MiniBooNE experiment. . . . . . . 21

2.3 Allowed $\left(\Delta m_{41}^{2}, \Delta m_{51}^{2}\right)$ regions for fits to $(3+2)$ models. . . . . . . . . . . 22

3.1 Scheme of the detector positions in the short baseline at Fermilab. . . . . . . 24

3.2 Oscillation probability behaviour with distance travelled for different values of $\Delta m^{2}$ at fixed energy $E_{\nu}=700 \mathrm{MeV}$ and $\sin ^{2} 2 \theta=0.1 \ldots \ldots 24$

3.3 SBN sensitivity to $\nu_{\mu} \rightarrow \nu_{e} \ldots \ldots \ldots \ldots \ldots \ldots \ldots$

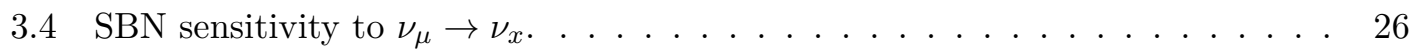

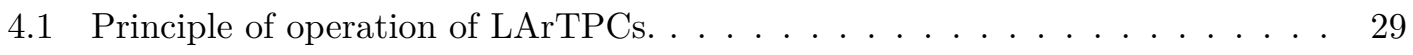

4.2 Neutrino interaction in the ArgoNeuT detector. . . . . . . . . . . . . . 30

4.3 Excitation and light emission in liquid argon. . . . . . . . . . . . . 30

4.4 Relative light intensity and collected charge as a function of the electric field in the TPC . . . . . . . . . . . . . . . . . . . . 31

4.5 Kinetic energy loss as a function of the total range for different ionising particles. 32

5.1 Housing of the TPC in the cryostat and TPC with APAs and CPAs. . . . . . 33

5.2 Photo of the SBND building. . . . . . . . . . . . . . . . . 34

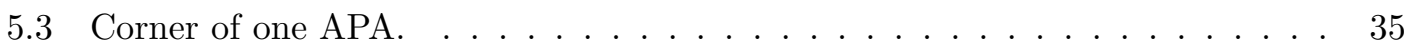

5.4 Rendering of the $\mathrm{U}, \mathrm{V}$ and $\mathrm{Y}$ planes with crossbars and combs. . . . . . . 36

5.5 Photo of region of the inside of the field cage . . . . . . . . . . 36

5.6 Schematic of the LArIAT beam line detectors. . . . . . . . . . . . . . . . 38

5.7 Schematic of the LArIAT detectors. . . . . . . . . . . . . . . . . . 39

5.8 Photo of the LArIAT cryostat. . . . . . . . . . . . . . . . . . 39

5.9 Single photoelectron response in LArIAT. . . . . . . . . . . . . . . . . . . . . 41

6.1 Frequency as a function of the wire length. . . . . . . . . . . . . 44 
6.2 Distribution of wire lengths in the SBND detector after accounting for combs

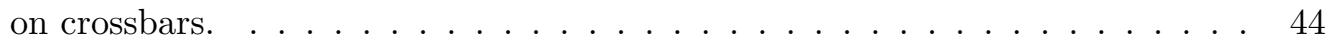

6.3 Dedicated laser tensioning device. . . . . . . . . . . . . . . . . . 45

6.4 Basic circuit scheme for a wire tension measurement using an electric circuit. 46

6.5 Expected signal with two resonant peaks of the electric wire tension measure-

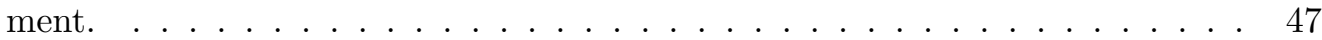

6.6 Example of the signal of two wires at different tensions with the fit. . . . . . 49

$7.14 \mathrm{~m}$ long frame with soldered wires. . . . . . . . . . . . . . . . . 51

7.2 Movable combs set-up. . . . . . . . . . . . . . . . . . . . 51

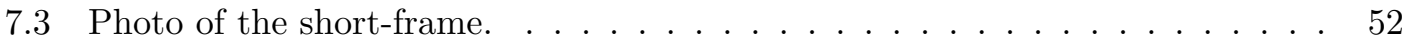

7.4 Test frame at Daresbury Laboratory. . . . . . . . . . . . . . . . . . . . . . 53

7.5 Weight used in the wire tensioning procedure. . . . . . . . . . . . 53

7.6 Drawing of a U-TOP geometry board circuit. . . . . . . . . . . . . . . . . 54

7.7 Copper-beryllium wires and readout cables soldered on the holding-signal PCB board. . . . . . . . . . . . . . . . . . . . . . . . . 55

7.8 Board connector. . . . . . . . . . . . . . . . . . 56

7.9 A screen-shot of the LabVIEW acquisition script. . . . . . . . . . . . . . . 57

7.10 Signal for wires soldered at two different tensions with constant bias of $1 \mathrm{kV}$. $\quad 59$

7.11 Linear baseline. . . . . . . . . . . . . . . . . . . . . . . . . 59

7.12 Instrumentation amplifier. . . . . . . . . . . . . . . . . . . . . 60

7.13 Noise before and after the addition of the instrumentation amplifier. . . . . . 60

7.14 Signal for wires soldered at $300 \mathrm{~g}$ and $500 \mathrm{~g}$ with a constant bias of $500 \mathrm{~V}$. . . 61

7.15 Cartoon explaining the method of operation of the $V 2$ version of the circuit . 62

7.16 Electric scheme of the $V 2$ circuit. . . . . . . . . . . . . . . . . . . . . . . . . 62

7.17 Comparison between beyond and below the SBND voltage limit for $1.5 \mathrm{~m}$ long segments.

7.18 Comparison between beyond and below the SBND voltage limit for $1.0 \mathrm{~m}$ long segments.

7.19 Comparison between beyond and below the SBND voltage limit for $0.75 \mathrm{~m}$ long wires. . . . . . . . . . . . . . . . . . . . . . 6 64

7.20 Resolution of the method for repeated measurements. . . . . . . . . . . . . 66

7.21 Laser set-up used to calibrate the wire tensioning measurement. . . . . . . . . 66

7.22 Laser light reflected on single wire. . . . . . . . . . . . . . . . . . . 67

7.23 DAQ script for laser tensioning set-up. . . . . . . . . . . . . . . 67 
7.24 Calibration of laser measurements. . . . . . . . . . . . . . . . 68

7.25 Bias of laser measurements as a function of wire length. . . . . . . . . . . . 69

7.26 Calibration of electric measurements with respect to laser measurement. . . . 70

7.27 Tension bias between the electric and the corrected laser measurements. . . . 71

7.28 Cold test set-up. . . . . . . . . . . . . . . . . . . . . . . 72

7.29 Temperature vs. time plot for tension measurement in cold. . . . . . . . . . . 73

7.30 Frequency vs. temperature measured during the cold test. . . . . . . . . . . . 74

7.31 Tension vs. temperature measured during the cold test. . . . . . . . . . . . . 74

7.32 Schematic of the short-frame and reference system. . . . . . . . . . . . 75

7.33 Set-up for bonding technique tests. . . . . . . . . . . . . . . . . 77

7.34 Signal for two $72 \mathrm{~cm}$ long wires with slightly different resonant frequencies. . 78

7.35 Loss in tension after two weeks. . . . . . . . . . . . . . . . . . 78

7.36 Example of measurements with combs. . . . . . . . . . . . . . . . 79

7.37 Photos of set-up for 32 channel readout. . . . . . . . . . . . . . . . 80

7.38 Connections to the SBND board for electric wire tension measurement. . . . 80

7.39 Data of the first 8 channels of the 32 channel acquisition performed at Daresbury Laboratory with the SBND test frame. . . . . . . . . . . . . . . 81

7.40 Data of channel 8 to 16 of the 32 channel acquisition performed at Daresbury Laboratory with the SBND mock-up frame. . . . . . . . . . . . . . . 82

8.1 Average number of detected photons per $\mathrm{MeV}$ along the centre of the TPC as a function of the distance from the photocathode plane in the configuration with WLS-covered reflector foils covering the cathode. . . . . . . . . . . . 85

8.2 Result of the electron drift time with glued reflector foils. . . . . . . . . . . 87

8.3 Result of the electron drift time without glued reflector foils. . . . . . . . . . 87

8.4 Set-up with small frame, mesh and foils. . . . . . . . . . . . . . . . 88

8.5 Two DF2000MA foils glued back-to-back shown after thermal cycling test. . . 90

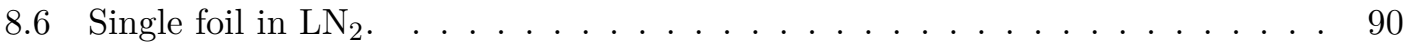

8.7 Folds on foils after cold-test. . . . . . . . . . . . . . . . . . . . . . 91

8.8 Photo of the evaporator installed in the clean room. . . . . . . . . . . . . 93

8.9 Photo of one crucible system installed on the bottom of the evaporator. . . . 94

8.10 Foil installed on rotating disk hung on the evaporator lid before and after the

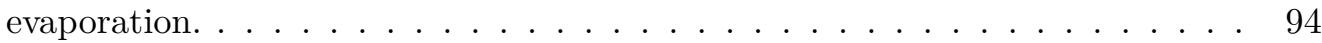

8.11 Inside bottom view of the evaporator. . . . . . . . . . . . . . . 95

8.12 Data from the evaporation sensor. . . . . . . . . . . . . . . . 97 


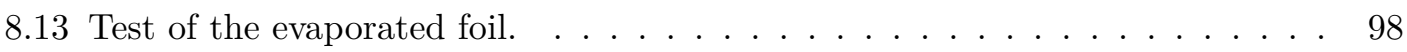

8.14 Evaporated foil after the dunk test in liquid nitrogen. . . . . . . . . . . 98

8.15 TPB density as a function of the distance from centre of the rotating disk. . . 99

8.16 Deposition thickness vs. distance from the centre. . . . . . . . . . . . . . . 100

8.17 Design of the SBND-like cathode installed in the LArIAT detector. . . . . . . 100

8.18 The LArIAT TPC with the mesh cathode installed. . . . . . . . . . . . . . . . 101

8.19 The LArIAT cathode with foils installed between layers of mesh. . . . . . . . 101

9.1 LArIAT beam structure. . . . . . . . . . . . . . . . . . . . . . . . . . . . 104

9.2 Data of single photoelectron rates taken by LArIAT in July 2016. . . . . . . . 105

9.3 Deposited energy distribution in $x, y, z$ adding the contribution of the ten spills simulated. . . . . . . . . . . . . . . . . . . . 106

9.4 Distribution of ion arrival time at the cathode. . . . . . . . . . . . . . 107 


\section{Abstract}

Liquid argon Time Projection Chambers (LArTPCs) are becoming widely used as neutrino detectors because of their image-like event reconstruction which enables precision neutrino measurements. They primarily use ionisation charge to reconstruct neutrino events. It has been shown, however, that the scintillation light emitted by liquid argon could be exploited to improve their performance. As the neutrino measurements planned in the near future require large-scale experiments, their construction presents challenges in terms of both charge and light collection. In this dissertation we present solutions developed to improve the performance in both aspects of these detectors. We present a new wire tensioning measurement method that allows a remote measurement of the tension of the large number wires that constitute the TPC anode. We also discuss the development and installation of WLS-compound covered foils for the SBND neutrino detector at Fermilab, which is a technique proposed to augment light collection in LArTPCs. This included preparing a SBND-like mesh cathode and testing it in the Run III of LArIAT, a test beam detector also located at Fermilab. Finally, we present a study aimed at understanding late scintillation light emitted by recombining positive argon ions using LArIAT data, which could affect large scale surface detectors. 


\section{Declaration}

No portion of the work referred to in the dissertation has been submitted in support of an application for another degree or qualification of this or any other university or other institute of learning. 


\section{Copyright Statement}

i. The author of this dissertation (including any appendices and/or schedules to this dissertation) owns certain copyright or related rights in it (the "Copyright") and s/he has given The University of Manchester certain rights to use such Copyright, including for administrative purposes.

ii. Copies of this dissertation, either in full or in extracts and whether in hard or electronic copy, may be made only in accordance with the Copyright, Designs and Patents Act 1988 (as amended) and regulations issued under it or, where appropriate, in accordance with licensing agreements which the University has from time to time. This page must form part of any such copies made.

iii. The ownership of certain Copyright, patents, designs, trade marks and other intellectual property (the "Intellectual Property") and any reproductions of copyright works in the dissertation, for example graphs and tables ("Reproductions"), which may be described in this dissertation, may not be owned by the author and may be owned by third parties. Such Intellectual Property and Reproductions cannot and must not be made available for use without the prior written permission of the owner(s) of the relevant Intellectual Property and/or Reproductions.

iv. Further information on the conditions under which disclosure, publication and commercialisation of this dissertation, the Copyright and any Intellectual Property and/or Reproductions described in it may take place is available in the University IP Policy (see http://documents.manchester.ac.uk/DocuInfo.aspx?DocID=24420), in any relevant Dissertation restriction declarations deposited in the University Library, The University Library's regulations (see http://www.library.manchester.ac.uk/about/regulations) and in The University's policy on Presentation of Dissertations. 


\section{Acknowledgements}

First of all I would like to express my gratitude to my supervisor, Dr. Andrzej Szelc, for the support from the beginning to the end of the work presented in this dissertation and for the precious advice that I received from him, which guided me in the process of becoming less of a physics student and more of a physicist. I also want to show my appreciation to Dr. Diego Garcia-Gamez for the endless help and suggestions on every aspect of my work throughout the year, including this dissertation. I extend my thanks to all the technical staff in the Schuster building and especially to Michael Perry and Claire Fuzipeg for all their advice and knowledge on technical features that they shared with me. A particular recognition is also due to Prof. Stefan Söldner-Rembold, Dr. Yvonne Peters, Dr. Justin Evans and, again, Dr. Andrzej Szelc for the valuable discussions and advice regarding the future of my career in physics. I also say thank you to Vincent Basque and Brían Ó Fearraigh for suggestions in the writing of this dissertation.

Finally, I am grateful to my mother, my father and my sister. Thanks for the tireless constant support throughout this year and for making this possible. 


\section{Chapter 1}

\section{Introduction}

Neutrino physics is one of the quickest developing areas of particle physics and it is one of the windows of opportunity to understand and measure new phenomena beyond the Standard Model of Particle Physics. Since the discovery of neutrino oscillations, predicted by Bruno Pontecorvo in 1957, it has been necessary to modify the Standard Model to accommodate the non-zero mass neutrinos and the violation of the lepton number conservation. The parameters of these oscillations have now been measured in a number of experiments. Chapter 2 of this dissertation discusses neutrino physics, neutrino interactions and describes the mechanism of neutrino oscillation. It also summarises the main results of the measurements of oscillation parameters and the open questions in neutrino physics. Focusing on the description of "anomalies" found in oscillation experiments, the hypothesis of the existence of a non-weakly-interacting ("sterile") neutrino, that could account for the unexpected results, is also presented. Chapter 3 describes the Short Baseline Neutrino (SBN) program, a set of neutrino experiments specifically designed to address and resolve the hypothesis of the "sterile" neutrino, and focuses on the main physics goals of the Short Baseline Near Detector (SBND), one of its detectors. The SBN program is the first combination of experiments employing three different Liquid Argon Time Projection Chambers (LArTPCs). Proposed by Carlo Rubbia in 1977, they are a relatively new type of detector that is becoming extensively used in neutrino physics thanks to their excellent position and energy resolution. The description of LArTPC detectors is the object of Chapter 4. Besides validating the sterile neutrino hypothesis, the SBN program is also a test-bed for the development of LArTPC detectors. The present dissertation is focused on R\&D for the SBND detector, thus Chapter 5 technically describes its main features. Some of the new features for SBND have been tested in LArIAT, a small TPC detector used to test the behaviour of TPCs in a test beam. Therefore this chapter also outlines the main characteristics of the LArIAT TPC. 
One of the main component of LArTPCs are layers of thousands of wires that have to be tensioned at a precise value during detector construction. Charged particles resulting from the interactions of neutrinos with liquid argon, ionise the argon. The electrons resulting from the ionisation, drifted with a constant external electric field, induce a signal on the wires. It is crucial for the correct functioning of the detector that the wires maintain the nominal tension. Chapter 6 and Chapter 7 are dedicated to the description of a novel technique that measures the tension of wires by means of an electric circuit. This technique provides a quick, precise and non-invasive method to simultaneously test the tension of a large number of wires and has been developed specifically for the needs of the SBND experiment as a part of the work for this dissertation.

In liquid argon, a fraction of the ion-electron pairs can recombine emitting light that can be exploited to improve the performance of LArTPC detectors. A solution proposed to enhance the collection of light inside the detector is the installation of reflector foils coated with a wavelength-shifting compound. This has been proposed for the SBND detector. The development of this proposal, specifically the coating procedure, the choice of the suitable type of foils, the installation details and the test of the system in the LArIAT detector, is discussed in Chapter 8.

Some operating LArTPCs have observed more random light signals than expected. This effect could be ascribed to positive argon ions resulting from ionisation that recombine with electrons while drifting in the external electric field. Chapter 9 shows a preliminary study that compares scintillation light data from the LArIAT detector with expectations from Monte Carlo simulations of beam and cosmic ray interactions in the TPC. The study investigates whether the observed light could be a consequence of the drifting positive ions recombining at the detector cathode.

Finally, Chapter 10 summarises the main results discussed in the dissertation.

My work in this dissertation consisted of building the different wire set-ups, performing the measurements and calibrations and analysing the data. I developed the fitting procedure and analysis code used for the measurements. To develop the necessary hardware and acquisition software, I worked with technical staff and engineers and debugged any new versions. An important part of the project was assembling the evaporator used to coat the reflector foils with the wavelength-shifter compound and finalizing the procedure of evaporation. I used these procedures to evaporate the foils used in a run of the LArIAT detector. I have performed or, in the case of the MTS tests, followed the tests performed to select the foil substrate. I have followed the design and construction of the LArIAT cathode. To perform 
the preliminary study of late scintillation light in LArIAT, I updated a dedicated simulation module called "LArIATFileGen" to work with the G4beamline simulation framework. I wrote an analysis module to reconstruct the shape of the energy distribution in the chamber from the beam line simulation I ran and the available cosmic ray MC sample. Finally, I simulated the effects of ion propagation on scintillation light with a small dedicated simulation. 


\section{Chapter 2}

\section{Neutrino Physics}

\section{$2.1 \quad$ Neutrinos}

Neutrinos are among the most abundant particles in the Universe, yet they are among the least understood. Their detection is very challenging because they are weakly interacting, i.e. the probability of the interaction to occur is much smaller compared to that of strong or electromagnetic processes. This is a consequence of the relatively high mass of the Standard Model $W^{ \pm}(80 \mathrm{GeV})$ and $Z(91 \mathrm{GeV})$ bosons, carriers of the weak interaction, that suppresses the probability of their exchange. Within the Standard Model there exist three types (flavours) of neutrinos, each of them is associated to the corresponding lepton:

$$
\left(\begin{array}{l}
e^{-} \\
\nu_{e}
\end{array}\right) \quad\left(\begin{array}{l}
\mu^{-} \\
\nu_{\mu}
\end{array}\right) \quad\left(\begin{array}{l}
\tau^{-} \\
\nu_{\tau}
\end{array}\right)
$$

We know that there are three weakly interacting neutrinos because of the measurements of the decay width of the $Z$ boson at the LEP collider [1] which measured the number of active neutrinos,

$$
N_{\nu}=2.9840 \pm 0.0082
$$

consistent with three.

\subsubsection{Neutrino interactions}

Neutrino interactions can only be mediated by the $W$ boson (Charged Current) or the $Z$ boson (Neutral Current). Table 2.1 shows Feynman diagrams for the neutrino interaction vertices available in the Standard Model.

Charged Current (CC) interaction converts a (anti-)neutrino into the corresponding charged (anti-)lepton, while in Neutral Current (NC) interactions a (anti-)neutrino simply 
Table 2.1: Feynman diagrams of neutrino interaction vertices.

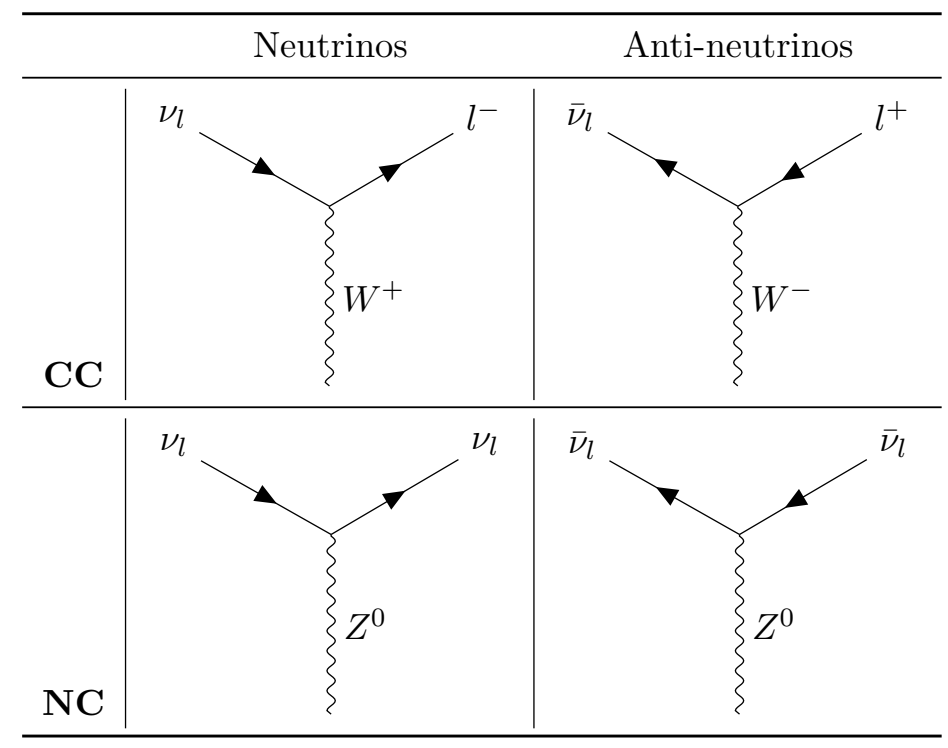

exchanges a $Z$ boson with a different particle and remains an (anti-)neutrino.

\subsection{Neutrino oscillation}

Neutrinos are created and detected in one of the three flavour states: $\nu_{e}, \nu_{\mu}$, and $\nu_{\tau}$. It has been experimentally established that neutrinos can change their flavour when travelling through vacuum or matter. This has been attributed to neutrino oscillation thanks to the measurements of Super-Kamiokande [2], SNO [3] and KamLAND [4] experiments. The Super-Kamiokande experiment, a water Cherenkov detector, showed data consistent with atmospheric $\nu_{\mu}$ oscillations into $\nu_{\tau}$ at $90 \%$ confidence level [2]. The Sudbury Neutrino Observatory (SNO), a heavy water Cherenkov detector, provided compelling evidence of solar neutrino oscillations measuring a non-zero non-electron component in neutrinos coming from the sun [3]. Finally, the KamLAND experiment detected $\bar{\nu}_{e}$ from nuclear reactors through inverse $\beta$-decay, $\bar{\nu}_{e}+p \rightarrow e^{+}+n$, and was able to confirm neutrino oscillations measuring disappearance of $\bar{\nu}_{e}[4]$.

In the oscillation model, neutrinos flavour states are a linear combination of three eigenstates of the free Hamiltonian $\left(\nu_{1}, \nu_{2}\right.$ and $\left.\nu_{3}\right)$ with coefficients given by the Pontecorvo-MakiNakagawa-Sakata (PMNS) matrix [5]:

$$
\left(\begin{array}{c}
\nu_{e} \\
\nu_{\mu} \\
\nu_{\tau}
\end{array}\right)=M_{\mathrm{PMNS}}\left(\begin{array}{c}
\nu_{1} \\
\nu_{2} \\
\nu_{3}
\end{array}\right)=\left(\begin{array}{ccc}
U_{e 1} & U_{e 2} & U_{e 3} \\
U_{\mu 1} & U_{\mu 2} & U_{\mu 3} \\
U_{\tau 1} & U_{\tau 2} & U_{\tau 3}
\end{array}\right)\left(\begin{array}{c}
\nu_{1} \\
\nu_{2} \\
\nu_{3}
\end{array}\right) .
$$


Given the fact that the PMNS matrix must be unitary to preserve probability, only a single phase $\delta$ is allowed which is related to CP-violation in the neutrino sector: for example the probability of $\nu_{\mu} \rightarrow \nu_{e}$ could be different from the probability of $\bar{\nu}_{\mu} \rightarrow \bar{\nu}_{e}$, indicating a violation of charge conjugation and parity symmetries. The PMNS matrix can be parametrised in the following way:

$$
M_{\mathrm{PMNS}} \equiv\left(\begin{array}{ccc}
c_{12} c_{13} & s_{12} s_{13} & s_{13} e^{-i \delta} \\
-s_{12} c_{23}-c_{12} s_{23} s_{13} e^{i \delta} & c_{12} c_{23}-s_{12} s_{23} s_{13} e^{i \delta} & s_{23} c_{13} \\
s_{12} s_{23}-c_{12} c_{23} s_{13} e^{i \delta} & -c_{12} s_{23}-s_{12} c_{23} s_{13} e^{i \delta} & c_{23} c_{13}
\end{array}\right) \text {, }
$$

where $s_{i j}=\sin \theta_{i j}, c_{i j}=\cos \theta_{i j}$ and $\theta_{i j}$ are the so-called mixing angles. In most real world situations, the probability of oscillation can be simplified to that of two flavours, in which case it can be expressed as:

$$
P\left(\nu_{\alpha} \rightarrow \nu_{\beta}\right)=\sin ^{2}(2 \theta) \sin ^{2}\left(1.27 \frac{\Delta m_{i j}^{2}\left[\mathrm{eV}^{2}\right] L[\mathrm{~km}]}{E[\mathrm{GeV}]}\right)
$$

where

- $\theta$ is a mixing angle defined by the equation

$$
\left(\begin{array}{c}
\nu_{\alpha} \\
\nu_{\beta}
\end{array}\right)=\left(\begin{array}{cc}
\cos \theta & \sin \theta \\
-\sin \theta & \cos \theta
\end{array}\right)\left(\begin{array}{c}
\nu_{1} \\
\nu_{2}
\end{array}\right)
$$

- $\Delta m_{i j}^{2} \equiv m_{i}^{2}-m_{j}^{2}$ is the difference of the squared masses of the two neutrino mass states;

- $L$ is the distance travelled;

- $E$ is the neutrino energy.

From Equation 2.3, it is possible to see that neutrino oscillations imply that neutrinos have mass. The probability of oscillation would be identically zero if all neutrinos had null mass, given that the $\Delta m^{2}$ is related with the frequency of the oscillation. Equation 2.3 also shows that neutrino oscillation are only sensitive to differences of the mass squared and that the important parameter that steers the oscillation scale is the ratio $L / E$. The two-flavour approximation is experimentally very useful when deriving oscillation parameters from data, since it greatly simplifies the mathematical formulas.

Many different experiments have been established in order to measure the parameters of neutrino oscillation (see for example Daya Bay [6], MINOS [7] and T2K [8]). The measured values are summarised in Table 2.2 taken from [9]. 
Table 2.2: Oscillation parameters (taken from [9]). The value of the best fit and the range within $3 \sigma$ are shown. The values in brackets correspond to inverted hierarchy $\left(m_{3}<m_{1}<\right.$ $\left.m_{2}\right) . \Delta m^{2}$ is defined as $\Delta m^{2}=m_{3}^{2}-\left(m_{1}^{2}+m_{2}^{2}\right) / 2$ such that $\Delta m^{2}>0\left(\Delta m^{2}<0\right)$ if $m_{1}<$ $m_{2}<m_{3}\left(m_{3}<m_{1}<m_{2}\right)$. For the $\delta$ parameter, the range within $2 \sigma$ is given. The sign of $\Delta m_{21}^{2}$ is known from matter effect in the sun.

\begin{tabular}{lll}
\hline Parameter & Best fit & $3 \sigma$ \\
\hline \hline$\Delta m_{21}^{2}\left[10^{-5} \mathrm{eV}^{2}\right]$ & 7.37 & $6.93-7.97$ \\
$\Delta m^{2}\left[10^{-3} \mathrm{eV}^{2}\right]$ & $2.5(2.46)$ & $2.37-2.63(2.33-2.60)$ \\
$\sin ^{2} \theta_{12}$ & 0.297 & $0.25-0.354$ \\
$\sin ^{2} \theta_{13}, \Delta m^{2}>0$ & 0.0214 & $0.0185-0.0246$ \\
$\sin ^{2} \theta_{13}, \Delta m^{2}<0$ & 0.0218 & $0.0186-0.0248$ \\
$\sin ^{2} \theta_{23}, \Delta m^{2}>0$ & 0.437 & $0.379-0.616$ \\
$\sin ^{2} \theta_{23}, \Delta m^{2}<0$ & 0.569 & $0.383-0.637$ \\
$\delta_{C P} / \pi$ & $1.35(1.32)$ & $0.92-1.99(0.83-1.99)$ \\
\hline
\end{tabular}

One thing that remains unresolved is the ordering of absolute neutrino masses. While we know the sign of $\Delta m_{12}^{2}$ from matter effects of electron neutrino interactions in the sun, the sign of $\Delta m_{32}^{2}$ is still unknown. Figure 2.1 [10] shows the three mass neutrino eigenstates with the relative flavour composition in the case of normal $\left(m_{1}<m_{2}<m_{3}\right)$ and inverted $\left(m_{3}<m_{1}<m_{2}\right)$ hierarchy considering approximated values of the oscillation parameters measured experimentally. One can also see why the two-flavour approximation is relatively
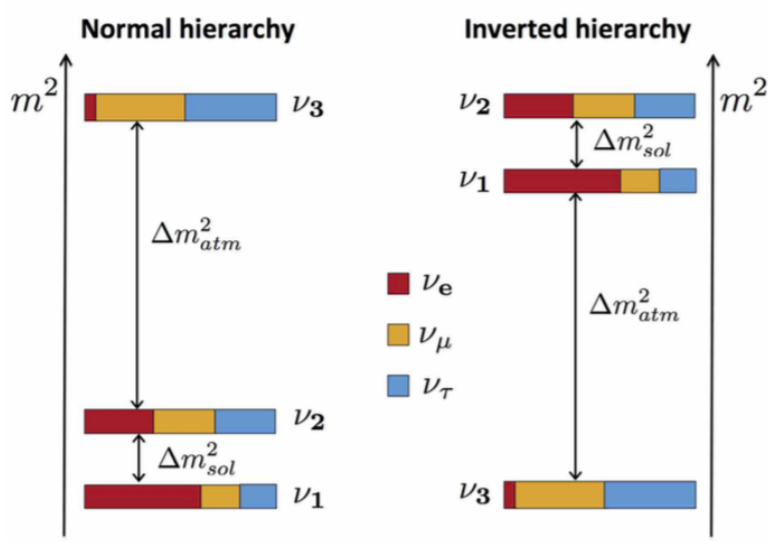

Figure 2.1: Flavour composition of mass eigenstates for normal and inverted hierarchy (taken from [10]). In this case $\Delta m_{\text {sol }}^{2} \equiv \Delta m_{12}^{2}$ and $\Delta m_{\text {atm }}^{2} \equiv \Delta m_{23}^{2}$ for the normal hierarchy and $\Delta m_{\text {sol }}^{2} \equiv \Delta m_{12}^{2}$ and $\Delta m_{\text {atm }}^{2} \equiv \Delta m_{31}^{2}$ for the inverted hierarchy.

precise. This is a result of the mass splittings among the three mass eigenstates being very different and the electron flavour hardly contributing to the third mass state as shown in Figure 2.1.

Knowing the mass hierarchy will be of importance in determining other oscillation parameters such as $\delta$, because of the ambiguity in decoupling matter effects and CP-violation. 
Future experiments can exploit matter effects in the earth on electron-neutrino component of beams to address the problem and will have the ability to determine the mass hierarchy and CP-violation phase without ambiguity (see for example the DUNE [11] and Hyper-K [12] experiments).

\subsection{Sterile neutrino}

Recently, experimental "anomalies" in short baseline experiments have been observed. These anomalies could be interpreted as oscillation to a fourth "sterile" neutrino. There are two different genres of anomalies: one is the larger-than-expected disappearance of electron anti-neutrinos from nuclear reactors [13] together with the "sources anomaly" observed by GALLEX [14] and SAGE [15] and the second is the excess of electron neutrinos from muon neutrinos from particle accelerator experiments [16, 17, 18, 19] ("LSND and MiniBooNE anomalies"). The LSND experiment aimed to search for $\bar{\nu}_{\mu} \rightarrow \bar{\nu}_{e}$ oscillation and found an unexpected event excess compatible with an oscillation to a heavy neutrino [16]. The MiniBooNE experiment was designed to address the LSND excess and found its own at a level of $2.8 \sigma$ [19]. Figure 2.2 shows the excess events found in the MiniBooNE data.

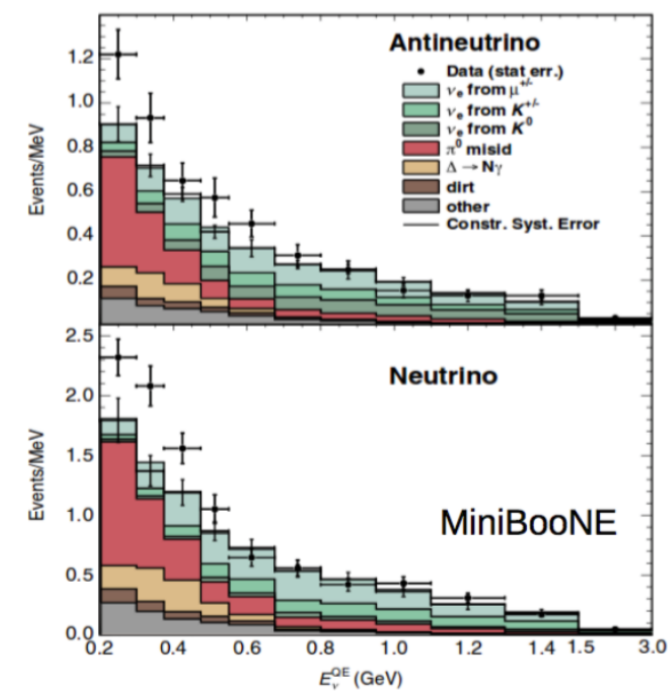

Figure 2.2: Electron-neutrino excess observed by the MiniBooNE experiment [19] in antineutrino (top) and neutrino (bottom).

These results can not be described with the current picture of Standard Model neutrinos because the mass splitting that would require, $\Delta m_{41}^{2} \approx[0.1-10] \mathrm{eV}^{2}$, is much larger than the already measured ones. A new neutrino state is needed to dominate the oscillations at small distances. This new particle would mainly be composed of a heavy neutrino $\nu_{4}$ and, 
since we have not seen it in $Z$ decay, it can only interact gravitationally, hence the name "sterile". Including the sterile neutrino in the Standard Model would lead to oscillation probabilities given by

$$
P_{\nu_{\alpha} \rightarrow \nu_{\beta}}^{3+1}=\delta_{\alpha \beta}-4\left|U_{\alpha 4}\right|^{2}\left(\delta_{\alpha \beta}-\left|U_{\alpha 4}\right|^{2}\right) \sin ^{2}\left(\frac{\Delta m_{41}^{2} L}{4 E_{\nu}}\right)
$$

where $U$ is a new $4 \times 4$ matrix, containing the PMNS matrix.

The existence of a fourth neutrino state should result in muon neutrino disappearance. Experiments such as MINOS, IceCube and others (see for example [20]) have not observed any deviations from predictions in this channel: this leads to a complicated and very interesting experimental situation. Global fits show incompatibilities between appearance and disappearance experiments and anti-neutrino and neutrino experiments. In general, it is found that a $(3+2)$ extension of the standard model can describe short baseline data (see [21] and [22]). Figure 2.3 shows the allowed $\left(\Delta m_{41}^{2}, \Delta m_{51}^{2}\right)$ regions for fits to $(3+2)$ models [21]. Models that theorise two additional neutrinos result in less tension between measurements than $(3+1)$ models, but, as there is no strong theoretical motivation to introduce a second sterile state, the $(3+1)$ model is often used to compare between experiments and we will follow this convention.
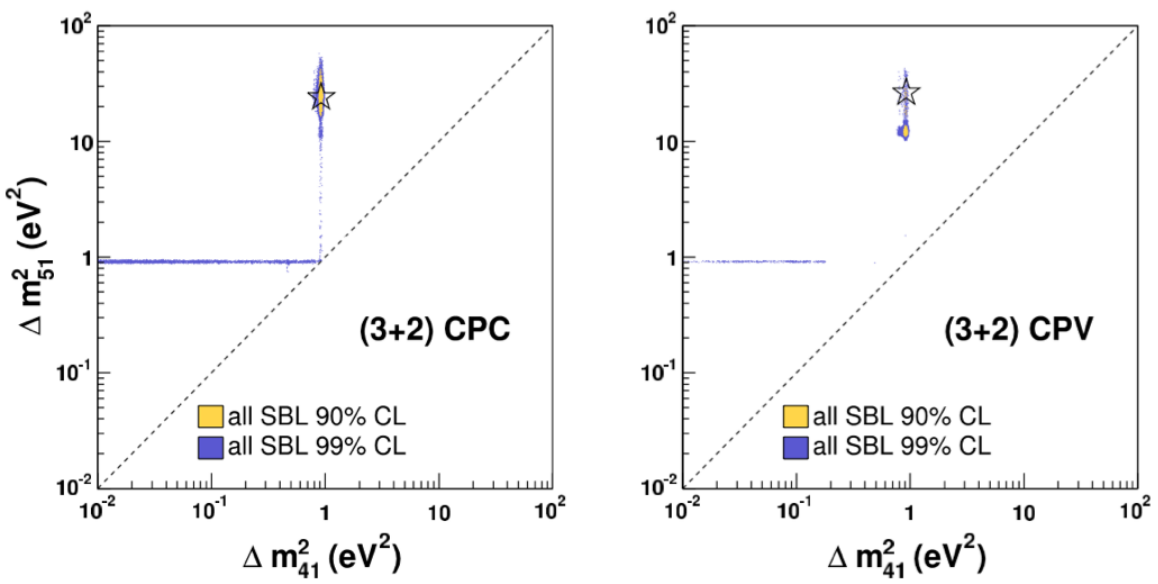

Figure 2.3: Allowed $\left(\Delta m_{41}^{2}, \Delta m_{51}^{2}\right)$ regions for $(3+2)$ oscillation models. Left: CP conserving model, right: $\mathrm{CP}$ violating model. Disappearance and appearance short-baseline data is included together with atmospheric constraints (taken from [21]).

New experiments are needed to finally resolve the anomalies [23]. There is a large experimental effort underway to resolve the experimental situation; one of such effort with one of the largest sensitivities will be the Short Baseline Neutrino (SBN) program, described in the next chapter. 


\section{Chapter 3}

\section{Short-Baseline Neutrino Program}

\subsection{Introduction}

The Short-Baseline Neutrino (SBN) [24] program consists of three Liquid Argon Time Projection Chambers (LArTPCs, see Chapter 4). The three TPCs will be located on-axis in the Booster Neutrino Beam (BNB) [24]. The neutrino beam originates from protons at $8 \mathrm{GeV}$ kinetic energy hitting a beryllium (Be) target [24]. The resulting secondary particles (mostly charged pions) are focused by a focusing horn that also selects the charge. The focused mesons propagate in the beam line decaying and emitting neutrinos. The resulting average energy of the resulting neutrino beam is $700 \mathrm{MeV}$ [25] leading to a length-over-energy scale of the order of

$$
\frac{\left\langle L_{\nu}\right\rangle}{\left\langle E_{\nu}\right\rangle} \sim \frac{600 \mathrm{~m}}{700 \mathrm{MeV}} \sim \mathcal{O}(1 \mathrm{~km} / \mathrm{GeV})
$$

The detector baselines are designed to maximise the probability of observing oscillations into sterile neutrinos with $\Delta m_{41}^{2} \approx[0.1-10] \mathrm{eV}^{2}$ : the near detector (SBND) will be located $110 \mathrm{~m}$ from the beam target, the MicroBooNE detector is positioned at $470 \mathrm{~m}$ and the ICARUS detector is located at $600 \mathrm{~m}$. See Figure 3.1 for a scheme of the placements of the detectors along the baseline.

The probability of oscillation as a function of the distance travelled by the neutrino beam is shown in Figure 3.2 for four different values of $\Delta m^{2}$ at fixed energy $E_{\nu}=700 \mathrm{MeV}$.

The probability to observe a signal is always very small at the position of the near detector for $\Delta m^{2}=0.4 \mathrm{eV}^{2}$ and $\Delta m^{2}=1.1 \mathrm{eV}^{2}$. This means that the near detector is a good probe of the intrinsic beam composition. At higher $\Delta m^{2}$ values, the oscillation wavelength is much shorter than the baseline meaning that oscillation signals would be visible even in the near detector, leading to a more complicated experimental situation. 


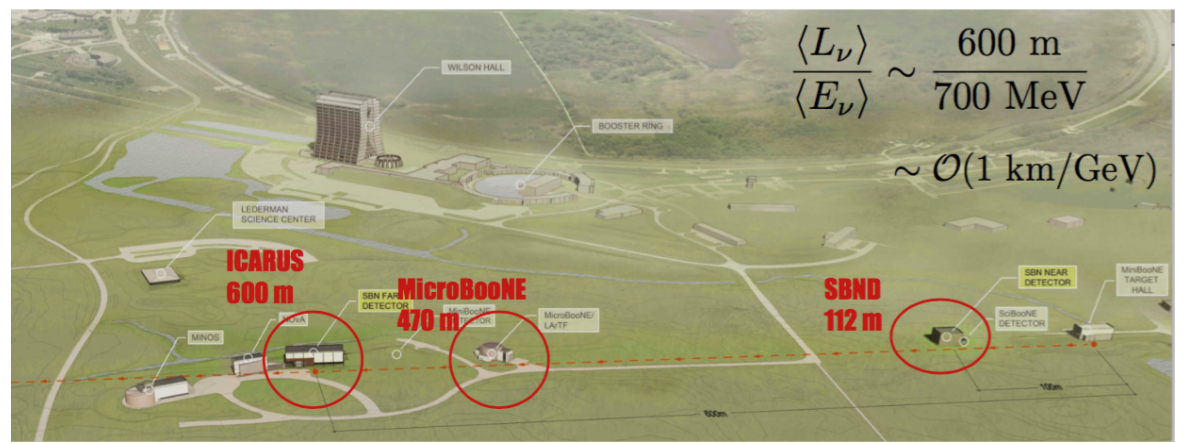

Figure 3.1: Scheme of the detector positions in the short baseline at Fermilab (taken from [24]). Beam travels from right to left along the red dashed line.

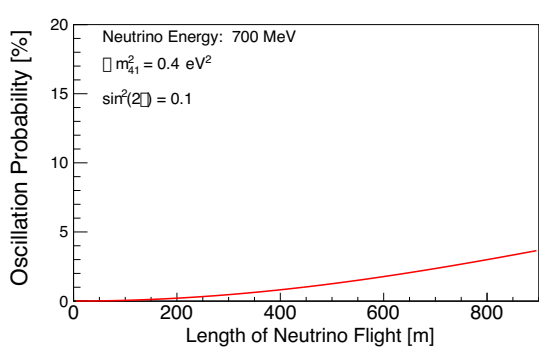

(a) $\Delta m^{2}=0.4 \mathrm{eV}^{2}$.

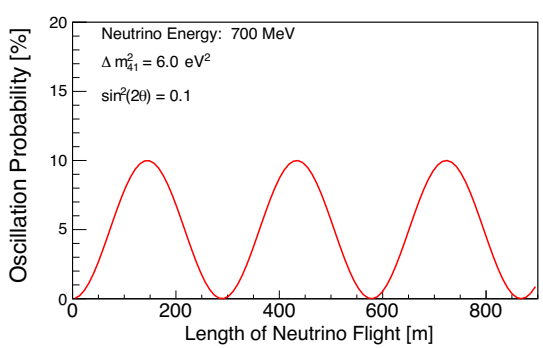

(c) $\Delta m^{2}=6 \mathrm{eV}^{2}$.

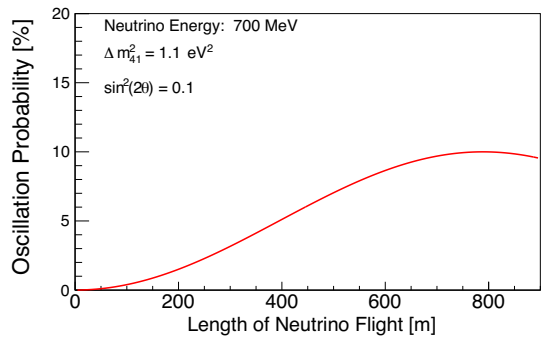

(b) $\Delta m^{2}=1.1 \mathrm{eV}^{2}$.

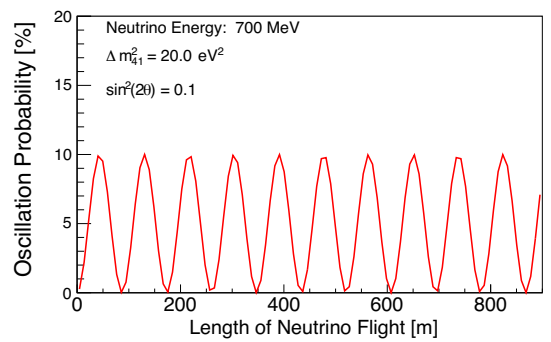

(d) $\Delta m^{2}=20 \mathrm{eV}^{2}$.

Figure 3.2: Oscillation probability behaviour with distance travelled for different values of $\Delta m^{2}$ at fixed energy $E_{\nu}=700 \mathrm{MeV}$ and $\sin ^{2} 2 \theta=0.1$ (taken from [24]). 


\subsection{Sensitivity to sterile neutrinos}

The main objective of the SBN program is to perform very sensitive measurements of the MiniBooNE excess to search for high- $\Delta m^{2}$ neutrino oscillations. The three LArTPCs will allow for a highly precise measurement of $\nu_{\mu} \rightarrow \nu_{e}$ appearance and $\nu_{\mu} \rightarrow \nu_{x}$ disappearance [24]. In the minimal extension of the SM to include a fourth sterile neutrino, the relative probabilities to these processes are given by

$$
P_{\nu_{\mu} \rightarrow \nu_{e}}^{3+1}=\sin ^{2} 2 \theta_{\mu e} \sin ^{2}\left(\frac{\Delta m^{2} L}{4 E_{\nu}}\right)
$$

and

$$
P_{\nu_{\mu} \rightarrow \nu_{\mu}}^{3+1}=1-\sin ^{2} 2 \theta_{\mu \mu} \sin ^{2}\left(\frac{\Delta m^{2} L}{4 E_{\nu}}\right)
$$

where $L$ is the distance travelled by the neutrino and $E_{\nu}$ is its energy. The oscillation amplitudes are a combination of the elements of $4 \times 4$ matrix $U$ that takes into account the mixing of $\nu_{\mu}$ and/or $\nu_{e}$ with $\nu_{4}: \sin ^{2} 2 \theta_{\mu e} \equiv 4\left|U_{\mu 4} U_{e 4}\right|^{2}$ and $\sin ^{2} 2 \theta_{\mu \mu} \equiv 4\left|U_{\mu 4}\right|^{2}\left(1-\left|U_{\mu 4}\right|^{2}\right)$.

The sensitivity of the SBN program to $\nu_{\mu} \rightarrow \nu_{e}$ in the $\left(\Delta m_{14}^{2}, \sin ^{2} 2 \theta_{\mu e}\right)$ is shown in Figure 3.3 [24]. The plot presents the combination of parameters allowed from the LSND experiment with $90 \%$ and $99 \% \mathrm{CL}$ and the excluded region by the SBN program at $90 \%, 3 \sigma$ and $5 \sigma \mathrm{CL}$. The LSND allowed region is almost completely ruled out at $5 \sigma \mathrm{CL}$. The plot in Figure $3.4[24]$ shows the SBN sensitivity prediction for $\nu_{\mu}$ disappearance at $90 \%, 3 \sigma$ and $5 \sigma$ confidence level, together with the $90 \%$ CL of the combined SciBooNE and MiniBooNE analysis. The conclusion is that SBN will be able to extend the sensitivity by an order of magnitude beyond the SciBooNE and MiniBooNE limits [24]. Recent measurements from other experiments, such as MINOS [26] and IceCube [27], probe a similar parameter space.

The near detector, SBND, plays an important part in the SBN programme measuring the intrinsic $\nu_{e}$ component in the Booster Neutrino Beam before any oscillation; MicroBooNE and ICARUS will look for a $\nu_{e}$ excess using the SBND measurement as a reference. Similarly, SBND will determine the unoscillated $\nu_{\mu}$ flux and MicroBooNE together with ICARUS will search for a deficit in $\nu_{\mu}$ events. Having far and near detectors that use the same technology allows for reduction systematic uncertainties and has been successfully used in oscillation searches.

\subsection{Short Baseline Near Detector (SBND)}

The Short Baseline Near Detector (SBND) is a Liquid Argon Time Projection Chamber (LArTPC) designed to be the near detector in the Short Baseline Neutrino (SBN) program. 


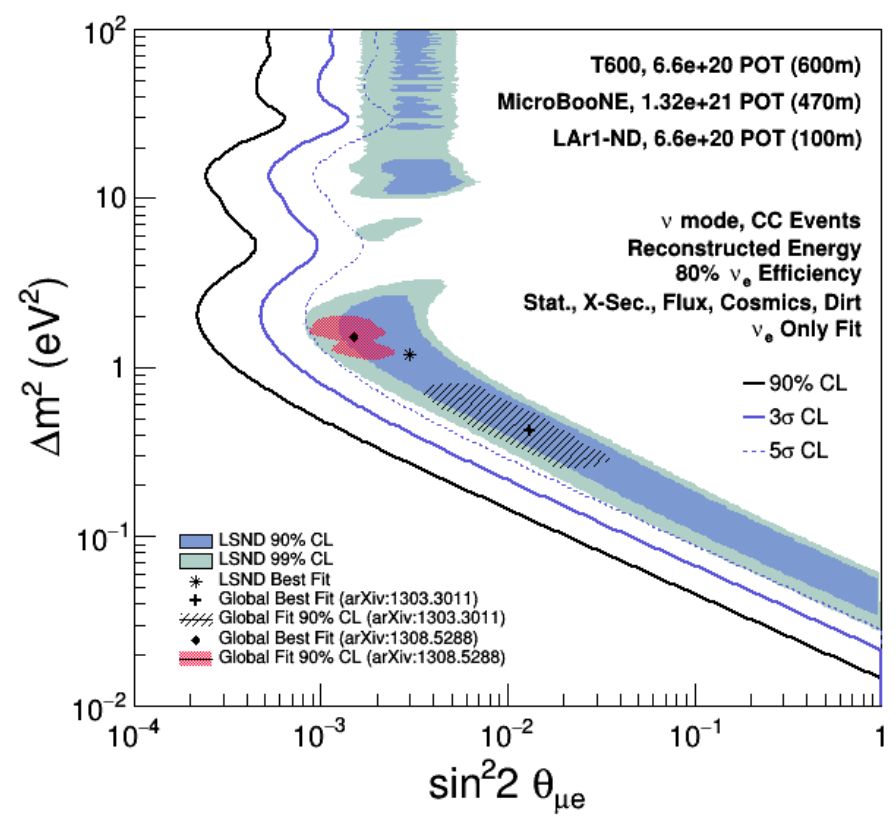

Figure 3.3: SBN sensitivity to sterile neutrino oscillation through the $\nu_{\mu} \rightarrow \nu_{e}$ channel [24]. LSND preferred area is also shown.

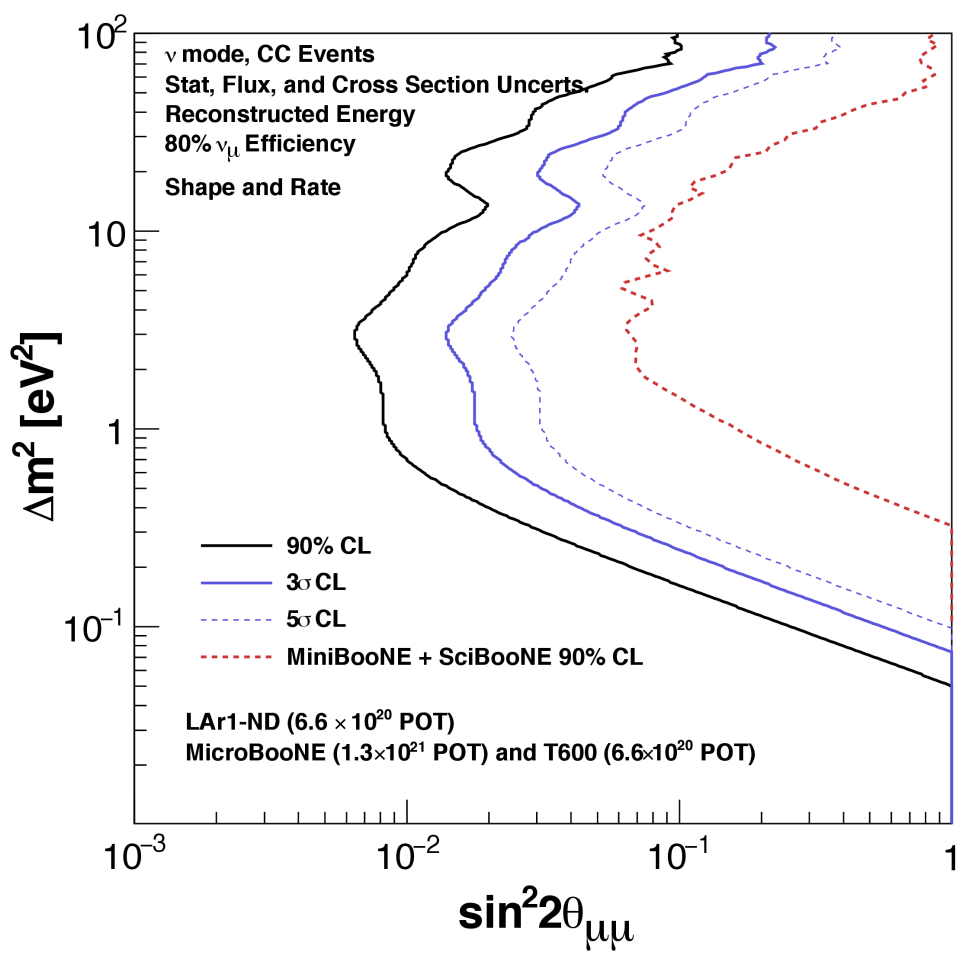

Figure 3.4: SBN sensitivity to sterile neutrino oscillation through the $\nu_{\mu} \rightarrow \nu_{x}$ channel [24]. The MiniBooNE + SciBooNE exclusion limit is also shown. 
It will be located at $110 \mathrm{~m}$ from the BNB target and, as it is not sensitive to oscillations for a large set of $\Delta m^{2}$, it will enable the detectors further down in the baseline to understand if an observed excess is due to oscillations or beam excess.

SBND, being the closest detector to the beam source, will observe the largest number of events: it will record 1.5 million $\nu_{\mu}$ and 12,000 $\nu_{e}$ charged current events per year [24]. This enables precise measurements of neutrino cross sections in argon. Knowing the cross section of neutrinos on Liquid Argon (LAr) is of fundamental importance for understanding and analysing oscillation data. So far, neutrino cross sections are a dominant systematic in oscillation experiments. The SBN program will exploit both the on-axis Booster Neutrino Beam and the off-axis Neutrinos at the Main Injector (NuMI) beam-line in the few hundred $\mathrm{MeV}$ to few $\mathrm{GeV}$ energy range to collect huge data sets of $\nu$-Ar interactions [24]. This will be also useful to test lepton universality since, if lepton universality holds, there should be no difference between $\nu_{e} \mathrm{CC}$ and $\nu_{\mu} \mathrm{CC}$ cross section [24].

The number of neutrinos in the detector allows precision measurements such as a measurement of $\sin ^{2} \theta_{W}$ that can be performed exploiting the channel $\nu_{\mu} e \rightarrow \nu_{\mu} e$. The event signature is an outgoing electron in the neutrino beam direction $(\cos \theta>0.99)$ with absence of recoil nucleon [24].

Finally, the SBND detector is also devised as a platform for testing new technologies for neutrino detectors such as discussed later in this dissertation. 


\section{Chapter 4}

\section{Liquid Argon Time Projection}

\section{Chambers}

\subsection{Introduction}

Liquid Argon Time Projection Chambers (LArTPCs), proposed in 1977 [28], are among the most promising detectors for neutrino physics. LArTPCs allow for excellent particle identification, calorimetric measurements and 3D event reconstruction. They have applications in both particle and astroparticle physics. They have been proposed for:

- studying neutrino oscillations on short and long accelerator baselines;

- detection of neutrinos coming from core-collapse Supernova explosions;

- searching for baryon number violating processes, e.g. proton decay;

- studying of atmospheric neutrinos.

\subsection{Principle of operation}

\subsubsection{Charge signal collection and reconstruction}

The LArTPC detects signals using the ionisation charge from charged particle tracks. Figure 4.1 shows the principle of operation. Ionising particles in the active volume resulting from neutrino interactions with Argon nuclei interact with argon creating electron-ion pairs. A constant uniform electric field makes the electrons created by the ionisation of charged particles drift towards the anode where they are collected. Cathode and anode are usually parallel to the direction of the beam $(\mathrm{Z})$ so that the electric field points in the transverse 


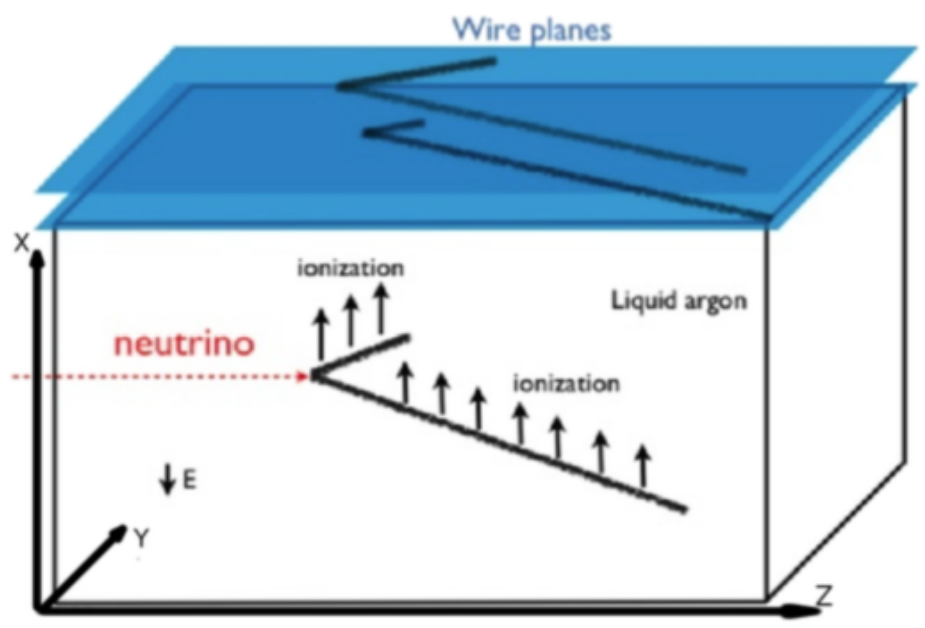

Figure 4.1: Principle of operation of LArTPCs and definition of the coordinate X, Y and $\mathrm{Z}[29]$.

direction $(\mathrm{X})$. The $\mathrm{Y}$ direction is defined to be perpendicular to both $\mathrm{X}$ and $\mathrm{Z}$. The anode is made of two or more parallel planes of wires, biased at different potentials. While the electrons are drifting, they induce an electric signal on the wires of different planes and are collected on the final plane. A 2D reconstruction (in the Y-Z plane) of the event is then possible by combining the coordinate of the wires on two different planes, which are at an angle between each other. A full 3D reconstruction is achieved by measuring the drift time of the charges: knowing the drift velocity in liquid argon given the electric field allows to reconstruct the $\mathrm{X}$ coordinate. LArTPCs also have excellent calorimetric properties, allowing for precise reconstruction of the energies deposited by particles by measuring the amount of collected charge.

The time projection chamber needs to be housed inside of a cryostat in order to maintain the temperature required to keep argon liquid $(87 \mathrm{~K})$. It needs to be kept stable within $1 \mathrm{~K}$ to prevent the electron drift velocity to change and to avoid excessive convective motion in the active volume [30].

LArTPC read-out systems operate as multi-channel waveform recorders [30] recording the signal induced on each wire. Data acquisition is performed by means of an ADC converter. Figure 4.2 shows a CC $\nu_{\mu}$ event in the ArgoNeuT TPC [31] as seen by the induction and collection wire planes [31]. The horizontal axis represents the wire coordinate while the vertical axis is the drift time in ticks. The colour of the tracks is proportional to the energy deposited reconstructed from the amplitude of the signal induced on the wires. In this example, the yellow track is a MIP escaping particle while the red line is a more highly ionising particle (e.g. proton). 

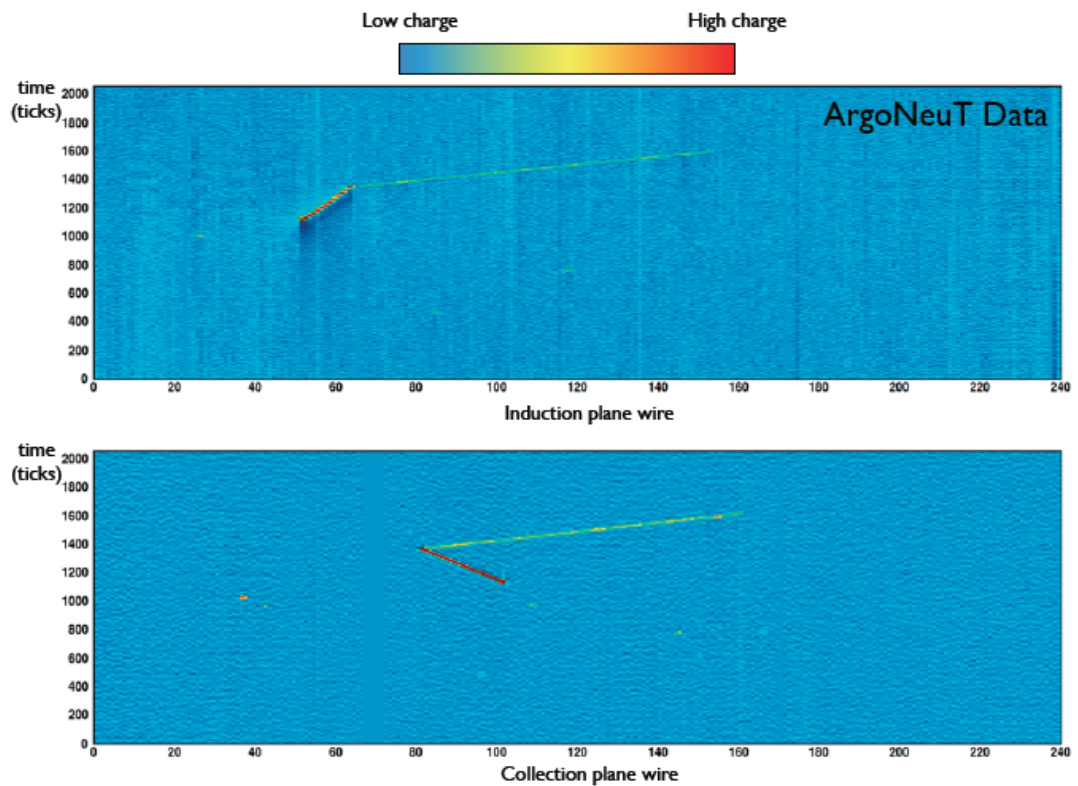

Figure 4.2: Neutrino interaction in the ArgoNeuT detector [31]. Event is shown as seen by the induction plane (top) and collection plane (bottom). The vertical coordinate is the drift time in ADC ticks while the horizontal axis is the wire coordinate on the respective plane.

\subsubsection{Scintillation light}

Apart from ionisation charge, particle interactions also cause scintillation light in liquid argon. Figure 4.3 shows the two typical channels of light emission in Liquid Argon. Light can result from both ionisation and excitation. In the latter case, an argon nucleus is excited, forms a molecule in an excited state and then emits light breaking the molecular bond. In the former case, the argon nucleus is ionised and bonds to form an ionised molecule. An electron can then be captured which results in an excited molecule that later de-excites emitting light. There are two intrinsic time scales of light emission: ns-scale ("fast component") and $\mu$ s-scale ("slow component").

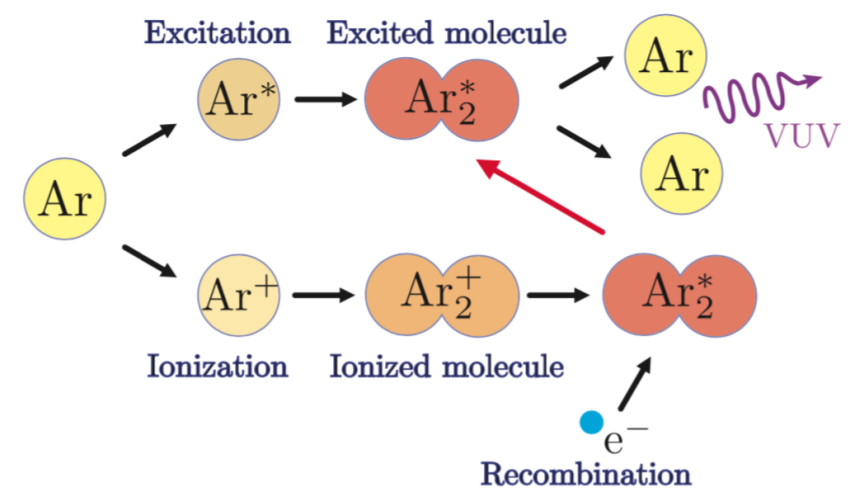

Figure 4.3: Excitation and light emission in liquid argon [32]. 
Liquid Argon emits 40000 photo-electrons per $\mathrm{MeV}$ at zero electric field [35]. This changes as a function of the electric field due to recombination: the higher the electric field, the less light will be emitted [24]. This is because the electric field makes the electrons and the ions drift towards anode and cathode respectively decreasing the chance for the recombination. A higher field lowers the probability of the recombination of the $\mathrm{e}^{-}$-ion pairs. As shown in Figure 4.4 [33], at the typical field strength of $500 \mathrm{~V} / \mathrm{cm}$, the energy deposition of a charged particle is divided almost equally between ionisation electrons and scintillation light: $2.9 \times 10^{4} \mathrm{e}_{\text {free }}^{-} / \mathrm{MeV}$ and $2.4 \times 10^{4} \gamma / \mathrm{MeV}$ [24].

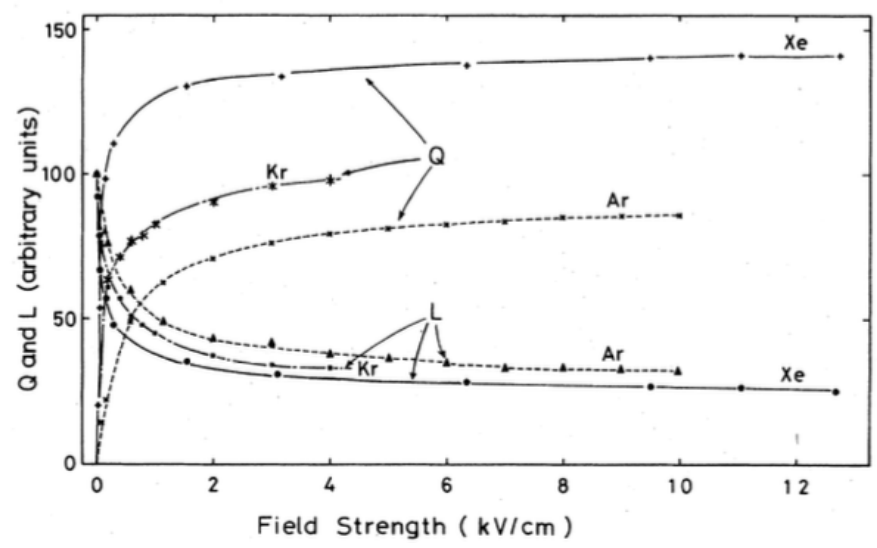

Figure 4.4: Relative light intensity (L) and collected charge (Q) as a function of the electric field in the TPC [33]. At the typical electric field strength, $500 \mathrm{~V} / \mathrm{cm}$, the energy deposited is almost evenly divided in ionisation electrons and scintillation photons.

A typical light collection system consists of arrays of photo-multipliers (PMTs) placed behind the anode planes to shield them from the electric field. Liquid argon emits vacuum ultra violet (VUV) light $(128 \mathrm{~nm})$ that is invisible to the PMTs due to the absorption in glass. For this reason, PMTs are often coated with a wavelength-shifter compound, for example tetraphenyl butadiene (TPB). In neutrino experiments liquid argon light is typically exploited as trigger of the event and to determine the electron drift start time, $t_{0}$, as the photons are much faster then the drifting charge [30]. Scintillation light patterns can be used to reject cosmic background, which is large in detectors constructed on the surface as the SBN detectors [34].

\subsection{Energy reconstruction and particle identification}

LArTPCs have excellent calorimetric capabilities. On average, the energy required to produce an electron-ion pair in LAr is $W_{\text {ion }}=23.6 \mathrm{eV}$ [31]. Counting the electrons collected 
by the anode, it is possible to measure the energy deposited by a charged particle. This requires accounting for recombination effects, which depend on the electric field and ionisation density [36] and change the number of electrons escaping from the interaction region. During the drift, these electrons can be attached by electronegative impurities $\left(\mathrm{O}_{2}, \mathrm{H}_{2} \mathrm{O}\right)$ which can further decrease the collected charge. The amount of these impurities is measured in LArTPCs using a quantity called "electron lifetime" and can be corrected for to extract the original deposited energy.

When charged particles stop in the active volume, energy deposition and track length can be combined to work out the stopping power. Stopping power is different for charged particles of different mass and charge and is therefore an excellent method for particle identification [31, 37]. Figure 4.5 conveys the deposited kinetic energy as a function of the total range [37].

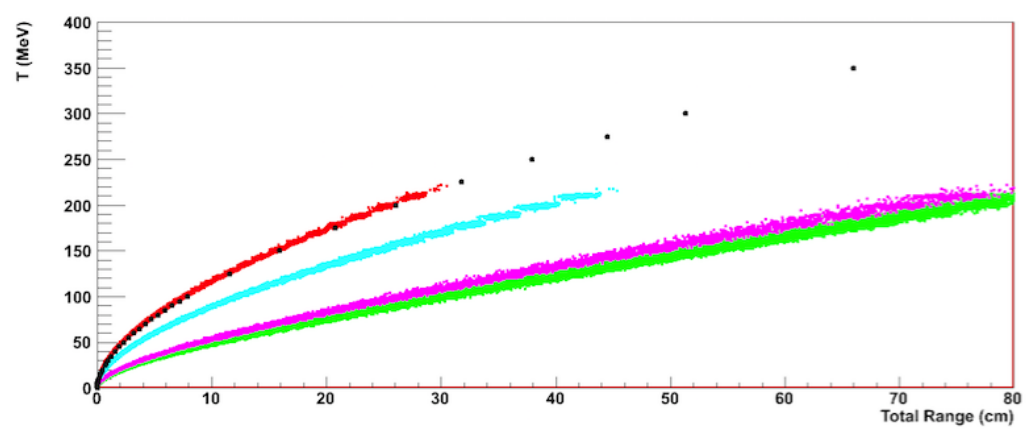

Figure 4.5: Kinetic energy loss as a function of the total range for different ionising particles: $\mu$ (green), $\pi$ (violet), $k$ (cyan) and $p$ (red) (taken from [37]). 


\section{Chapter 5}

\section{The SBND and LArIAT Detectors}

The work presented in this dissertation was performed in the framework of the SBND and LArIAT experiments. The present chapter illustrates the main characteristics of these detectors.

\subsection{The Short Baseline Near Detector (SBND)}

SBND will consist of two time projection chambers and will have a fiducial mass of $112 \mathrm{t}$ in an active volume of $5 \mathrm{~m} \times 4 \mathrm{~m} \times 4 \mathrm{~m}$. The drift areas will be housed in a cryostat and will be composed of two cathode plane assemblies (CPAs) and four anode plane assemblies (APAs). Moreover, in order to have a uniform electric field, a shaping field cage will be used. Figure 5.1 shows the housing of the TPC in the cryostat and a model of the TPC and Table 5.1 [24] summarises the key parameters of the SBND TPC.
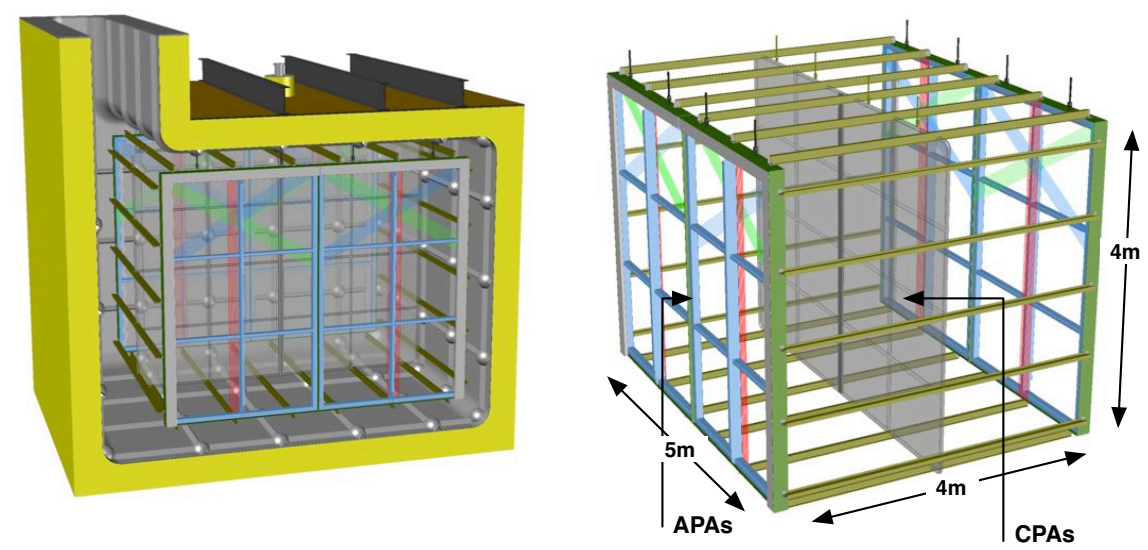

Figure 5.1: Housing of the TPC in the cryostat (left) and TPC with APAs and CPAs (right) [24].

The cryostat is an industrial membrane cryostat that is usually used for liquefied natural 
Table 5.1: SBND TPC key design parameters [24].

\begin{tabular}{lc}
\hline TPC Parameter & Value \\
\hline \hline TPC active volume & $5 \mathrm{~m}(\mathrm{~L}) \times 4 \mathrm{~m}(\mathrm{H}) \times 4 \mathrm{~m}(\mathrm{~W}), 112$ metric ton active LAr mass \\
Number of TPC cells & 2 drift volumes, $2 \mathrm{~m}$ drift length in each \\
Maximum drift time & $1.28 \mathrm{~ms}$ \\
Anode Plane Assembly & $2.5 \mathrm{~m} \times 4 \mathrm{~m}$ active area, with cold electronics mounted on 2 sides \\
Wire properties & $150 \mu \mathrm{m}, \mathrm{CuBe}$ \\
Wire planes & 3 planes on each APA, $\mathrm{U} \mathrm{V}$ at $\pm 60^{\circ}$ to vertical $(\mathrm{Y})$ \\
Cathode bias & $-100 \mathrm{kV}$ at $500 \mathrm{~V} / \mathrm{cm}$ drift field \\
Number of Wires & 2816 channels $/ \mathrm{APA}, 11264$ wires total in TPC \\
Wire tension & $0.5 \mathrm{~kg}$ at room temperature \\
\hline
\end{tabular}

gases [24]. The cryostat is a rectangular vessel with a neck and two planes on the top which contain the cold penetrations [24].

The cryostat and the TPC will be assembled in a building located $110 \mathrm{~m}$ from the Booster Neutrino Beam target (see Figure 5.2). The detector will be housed in the lower level while the higher level will be used for installation services and facilities.

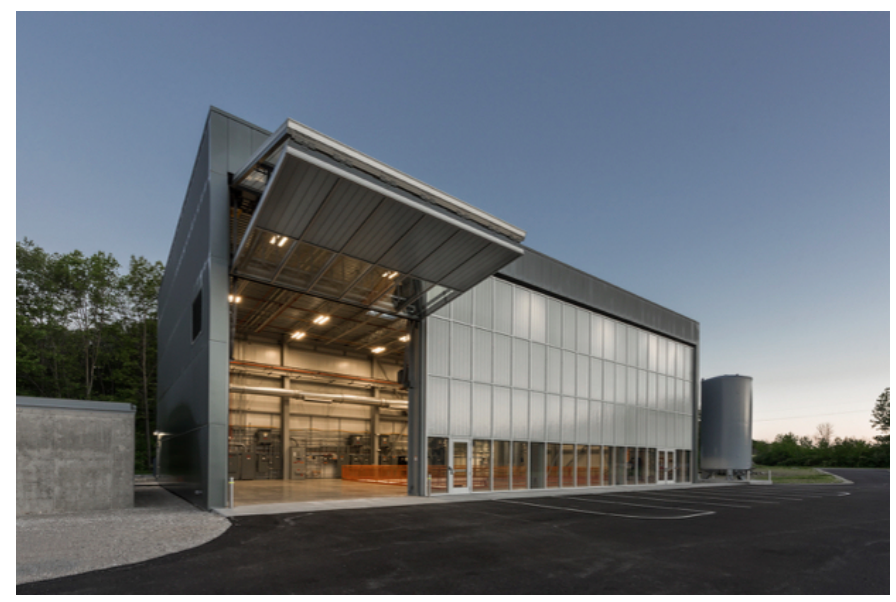

Figure 5.2: Photo of the SBND building (taken from [38]) at Fermilab.

\section{Cathode plane assembly}

The dimensions of each cathode plane assembly are $2.5 \mathrm{~m} \times 4 \mathrm{~m}$ [24]. The CPA is made of a stainless-steel framework while the openings of the frame could be either covered by stainless-steel sheets or by a transparent mesh plane [39]. The final choice will depend on the requirements of the light collection system (see Section 5.1.1).

In order to provide a field of $500 \mathrm{~V} / \mathrm{cm}$ with a drift length of $2 \mathrm{~m}$, the cathode planes need to be biased at $-100 \mathrm{kV}$. 


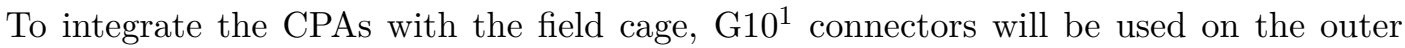
edges.

\section{Anode plane assembly}

The anode plane assemblies will have the same size of the CPAs [39] and will be constructed on a stainless-steel frame. Each of them will be composed of three planes (Y, U and V) oriented at different angles. Each plane is made of Copper-Beryllium (CuBe) wires of $150 \mu \mathrm{m}$ diameter, prepared at a nominal tension of $0.5 \mathrm{~kg}$ at room temperature. Wires are expected to get an additional $0.7 \mathrm{~kg}$ of tension when cooled down to liquid argon temperatures. The pitch between both wires and planes is $3 \mathrm{~mm}$. In the case of the collection plane $(\mathrm{Y})$, the wires are vertical, while the two induction planes, $\mathrm{U}$ and $\mathrm{V}$, have wires at an angle of $\pm 60^{\circ}$ from vertical (see Figure 5.3). Different planes are biased at different potentials in order to guarantee total transparency to the electrons drifting towards the outer plane (collection, Y). The biases for the planes $(\mathrm{U}, \mathrm{V}, \mathrm{Y})$ are $(-200 \mathrm{~V}, 0 \mathrm{~V}, 500 \mathrm{~V})$ respectively.

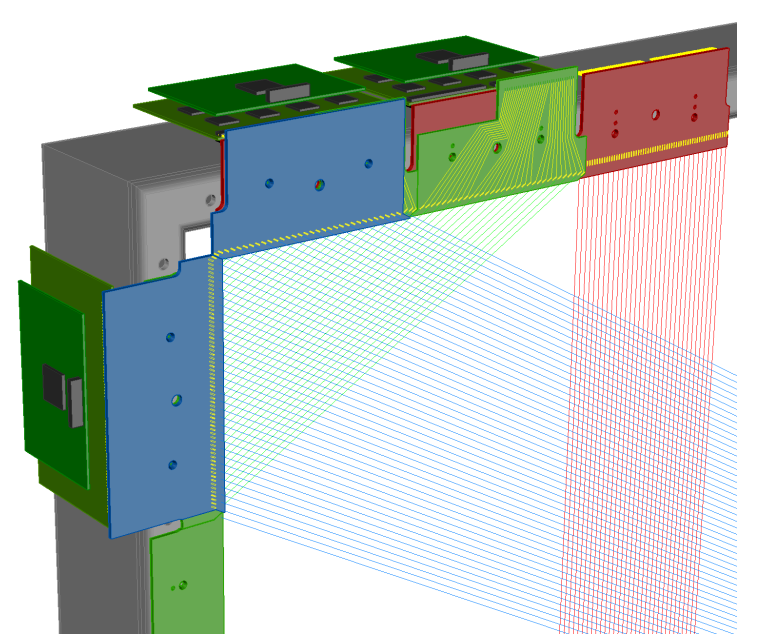

Figure 5.3: Corner of one APA showing different layers of wires and their orientation [24].

To limit the free length of the wires and maintain the wire plane spacing, "combs", plastic assemblies with slots for the wires, will be put on the crossbars of each APA [40]. Figure 5.4 shows combs for each of the APA plane.

Wires will be soldered to pads on geometry boards and will be also mechanically fixed by a bead of 2216 Grey Epoxy [40]. Voltage capacitors and resistors are required for each readout channel with a bias voltage (U and Y plane) [24]. For a more detailed discussion on wire tension and APA board resistors see Chapter 6 .

\footnotetext{
${ }^{1}$ Isolating material.
} 


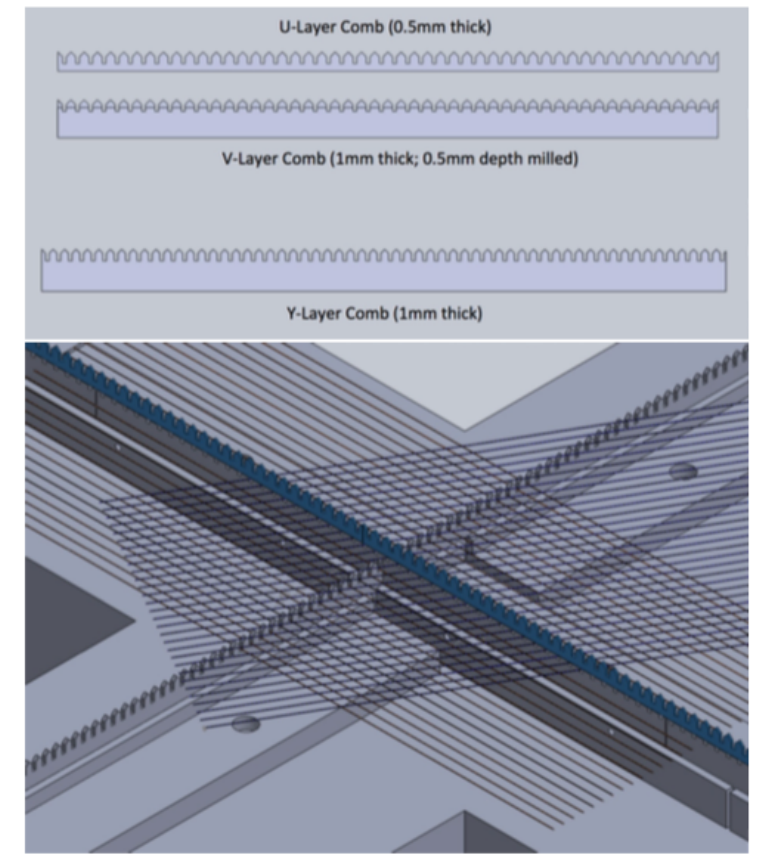

Figure 5.4: Rendering of the U, V and Y planes with crossbars and combs [40].

\section{Field cage}

The purpose of the field cage is to ensure the $500 \mathrm{~V} / \mathrm{cm}$ electric field is uniform throughout the entire TPC volume by gradually stepping the voltage from the CPA bias to the ground by means of a voltage divider. The cage will be made of $\mathrm{Cu}$ clad FR4 PCBs [24]. Figure 5.5 shows a picture of a region of the field cage seen from the inside.

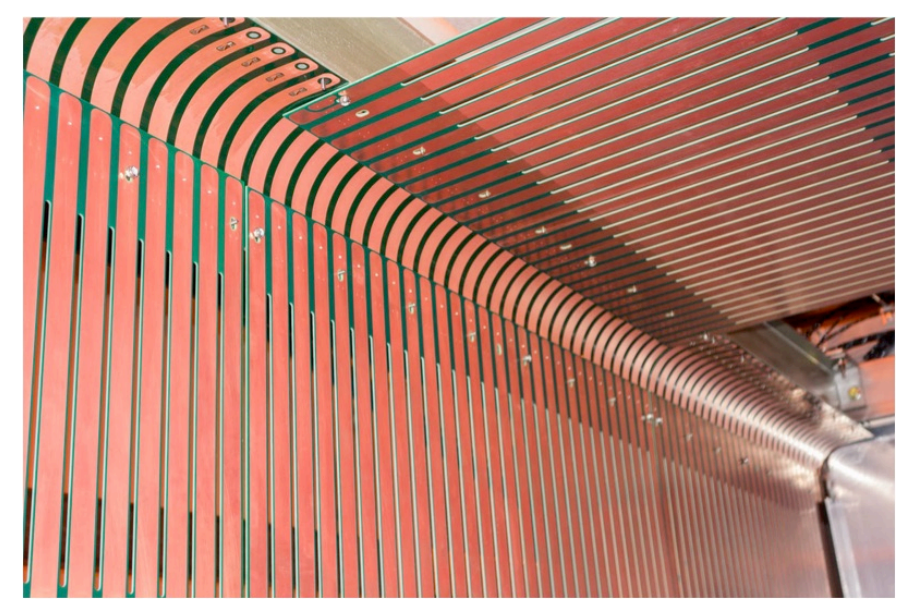

Figure 5.5: Photo of region of the inside of the field cage (taken from [24]). 


\subsubsection{Light collection system}

SBND is designed to have a high light yield light collection system. Light detection in SBND will be performed by means of 144 TPB-coated PMTs [24]. In addition, as a part of its mission to develop liquid argon light collection system technology, SBND will test other methods like light guide bars and reflective foils [24].

Light guide bars are thin $100 \mathrm{~cm} \times 2.54 \mathrm{~cm} \times 0.64 \mathrm{~cm}$ bars used to collect the VUV light. Photons entering in the light guide would be internally reflected towards the end of the bar, wavelength-shifted and detected by $3 \mathrm{SiPMs}$ [24]. It is expected that guide bars should provide an additional 20-30\% of light [35].

A light collection option under review is the installation of reflector foils coated with a wavelength shifter compound on the surface of the CPAs. They will naturally enhance the light yield of the detector. The foils are typically made of highly reflective and di-electric material and would have to be installed in a mesh cathode. Two layers of metallic mesh would keep the foils in position and allow enough transparency for light to be wavelength-shifted and reflected. The enhanced light collection efficiency and uniformity will help the measurement of low energy interactions like nuclear effects or supernova signals [35]. Developing the reflector foil component of the light collection system is part of the work exhibited in this dissertation (described in Chapter 8).

\subsection{Liquid Argon in A Test Beam (LArIAT)}

Liquid Argon in A Test Beam (LArIAT) is a Liquid Argon Time Projection Chamber whose main purpose is the calibration of the response of LArTPC detectors using a charged particle test beam [37]. The tests are conducted in the Fermilab Test Beam Facility [41] which provides low momenta particles in the range $0.2-20$. GeV. This range corresponds to charged particle resulting from a neutrino interaction in liquid argon [37] at accelerator neutrino energies. The response of the detector is under study and will benchmark the behaviour of LArTPCs for future experiments such as the SBN programme and DUNE [42]. Main physics goals are the study of the electron-gamma separation (important when looking for $\nu_{e}$ events), the muon sign determination (crucial to tell apart $\nu_{\mu}$ and $\bar{\nu}_{\mu}$ interactions) and calorimetric/particle identification calibration $[29,37]$. LArIAT is also the ideal set-up to measure the cross section of charged particles $(\pi, K)$ in liquid argon and to test new technologies. 


\subsubsection{Beam line}

A pion beam is created starting from a primary beam of protons with momentum $120 \mathrm{GeV} / c$ that hit a designated target during a spill lasting four seconds. The range of the pion energy is $8-32 \mathrm{GeV}$ [37]. These pions are then directed towards a tertiary target located in the MCenter building. This results in a tertiary beam that can also provide positive and negative electrons, muons and kaons at lower momenta down to $0.2 \mathrm{MeV} / c$ [37]. The tertiary line is composed of a target, a collimator system and bending magnets [37].

The tertiary beam line is instrumented with detectors aligned upstream and downstream of the TPC. These provide information about particle momenta, particle identification and the presence of beam halo [37]. See Figure 5.6 for a scheme of the beam detectors. The bending magnets and the wire chambers are exploited to measure particle momenta while the time of flight scintillators allow to tell apart the slower protons and kaons [43]. Muons and pions can be differentiated by means of the Cherenkov detector and the punch-through [43]. This

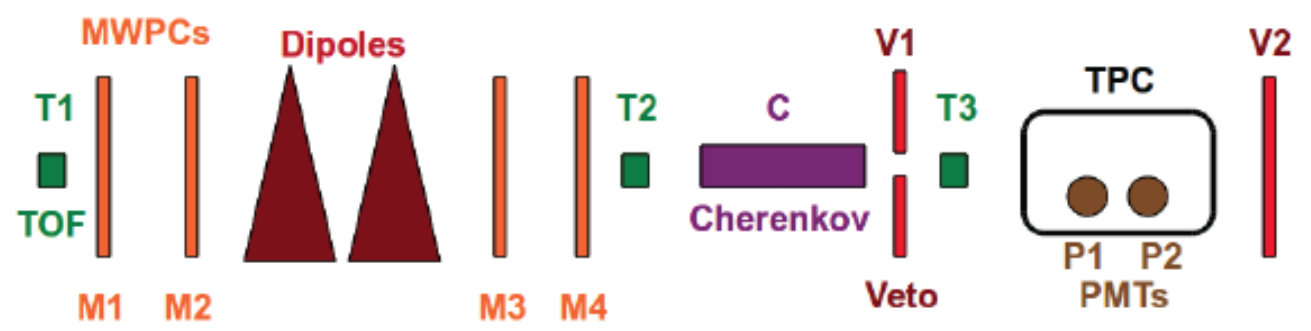

Figure 5.6: Schematic of the LArIAT beam line detectors. T1-3 are Time of Flight (TOF) plastic scintillator; $M 1-4$ are multi-wire proportional chambers; $C$ is a Cherenkov counter; $V 1-2$ are scintillator veto systems from beam halo and, in particular, $V 2$ is the punch-through system to detect through going particles [37].

system is able to provide triggers based on momentum selection and on particle identification.

\subsubsection{Detector}

The LArIAT TPC and cryostat are refurbished components of the ArgoNeuT (see [31]) detector [44]. A schematic of the detector is shown in Figure 5.7.

\section{Cryostat}

The cryostat is a stainless-steel cylinder whose main axis is parallel to the beam direction. It has a neck on the top to allow access of signal cables and HV feed-through [37], see Figure 5.8 for a photo.

The cryostat was slightly modified to include improvements and new features necessary 


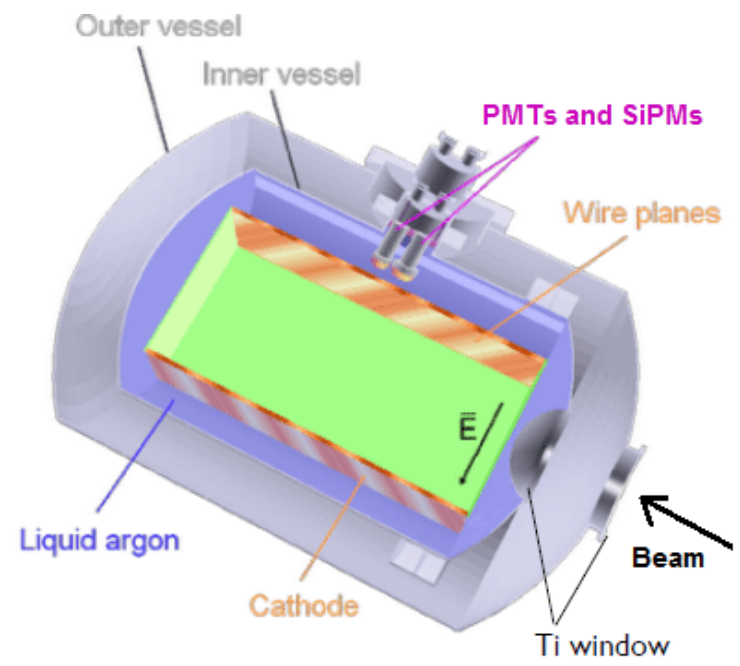

Figure 5.7: Schematic of the LArIAT detectors (taken from [44]). Outer and inner vessels are stainless-steel cryostats.

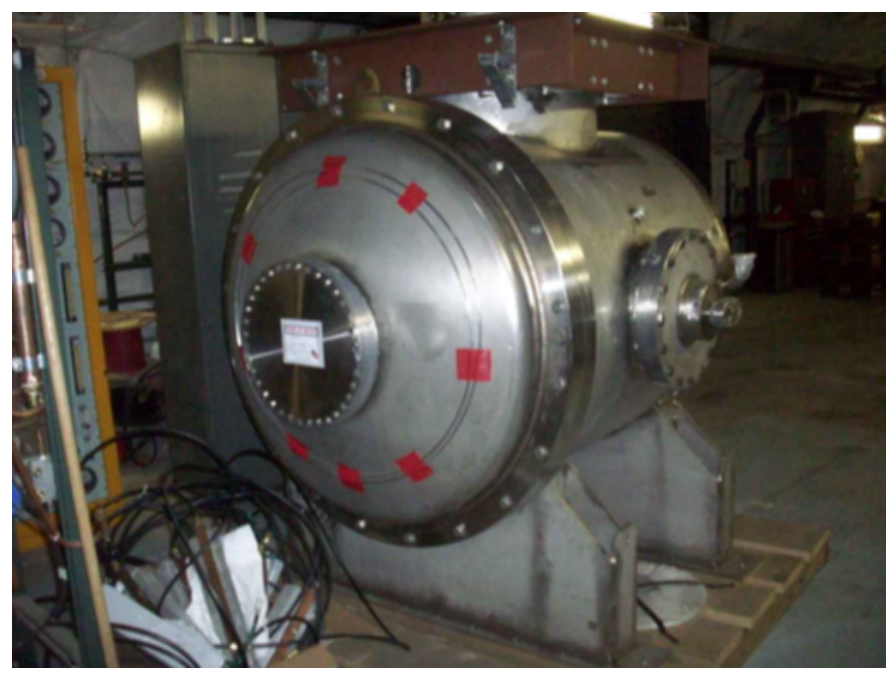

Figure 5.8: Photo of the LArIAT cryostat [29]. Size of the inner vessel is $76.2 \mathrm{~cm}$ diameter and $130 \mathrm{~cm}$ length. The front window is visible as well as the side flange for the light collection system installation. 
for the LArIAT experiment [37]:

- introduction of the beam window (front flange in Figure 5.8) to reduce the thickness of material that particles have to cross before getting in the TPC;

- new connections for argon purification and cooling;

- a side flange to install the light collection system.

TPC

The active volume is $w \times h \times l=47 \mathrm{~cm} \times 40 \mathrm{~cm} \times 90 \mathrm{~cm}$, resulting in $170 \mathrm{~L}$ of liquid argon delimited by a field cage that shapes the electric field [37].

The anode is made of three parallel planes of conducting wires. The inner layer is made of vertical non-instrumented wires whose purpose is to shape the field and to shield the outer planes from drifting charges. The central "induction" wire plane has wires oriented at $+60^{\circ}$ with respect to the beam direction, while the outer layer, "collection" plane, has wires oriented at $-60^{\circ}$ to allow positional reconstruction. The two outer planes are instrumented with cold readout electronics [37].

The field cage uses a set of field-shaping rings and a voltage divider to provide a constant and uniform electric field of $500 \mathrm{~V} / \mathrm{cm}$ from the cathode, a G10 layer with copper metallisation in the original design, to the anode [37].

\subsubsection{Light collection system}

The side flange of the cryostat houses the light collection system. Two different types of PMTs were employed (2" ETL PMT and 3" Hamamatsu R11065) plus two SiPM cryogenic detectors [29]. The collection and uniformity of light is enhanced by reflector foils (VIKUITY) installed in the inner side of the TPC. In order to detect the VUV photons emitted, the foil surfaces are covered by TPB, a wavelength shifter compound [37]. The signal produced by the photons detected in the light detectors is processed and digitised by an ADC CAEN V1751 sampling at $1 \mathrm{GS} / \mathrm{s}$ and therefore allowing to reconstruct single photoelectron peaks. To deduce the number of photons detected from the ADC counts, it is necessary to calibrate the response of the PMTs to a single photoelectron [45]. Figure 5.9 [46] shows an example of single photoelectron response obtained by counting single photoelectron peaks in the tail of the distribution of argon scintillation events. 


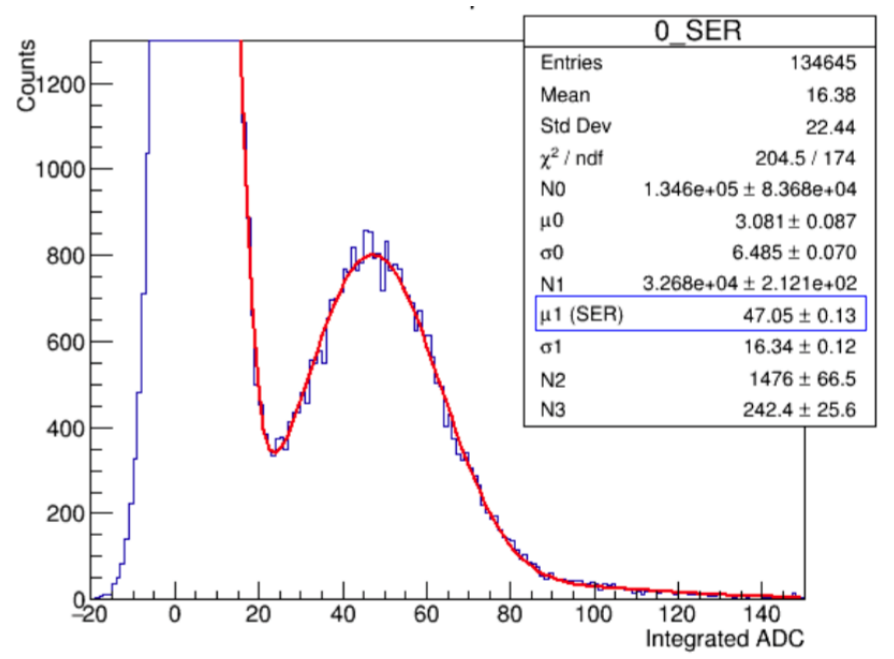

Figure 5.9: Single photoelectron response in LArIAT [46]. The horizontal axis represents the integrated ADC counts while the vertical axis denotes the corresponding occurrences. The noise manifests as a large peak at around 3 ADCs while the response to a photoelectron is estimated by the fit (red curve) to be at around 47 ADCs.

With the calibration in hand, it is possible to calculate the number of single photoelectrons collected by the light detectors in order to estimate the energy deposited, which is used in several ongoing analyses. 


\section{Chapter 6}

\section{Principles of an Electrical Wire}

\section{Tensioning Measurement for}

\section{LArTPCs}

In this and the following chapter a novel technique to measure wire tension is presented. It has been developed to measure the tension of the SBND Anode Plane Assemblies (APAs) during winding, but can be applied to commissioning of other future detectors like DUNE. In this chapter we describe the general principle of the measurement.

\subsection{Introduction}

Liquid argon time projection chambers use thousands of wires to collect the signals from ionisation charge drifting towards the anode. It is crucial for the successful operation of these detectors that all of these wires are at the same, or very close to, nominal tension. Overtensioned wires have a higher chance to break during the cool down, due to non-uniform contraction of the frame, boards, etc. at cryogenic temperatures. Broken wires might make large areas of the detector blind due to potential shorts. On the other hand, under-tensioned wires are also undesirable. Most of the wires are at an angle with respect to the vertical direction; as a consequence, gravity can make them sag if the tension is too low. In the extreme case, a sagging wire could touch an adjacent wire altering the signal of both. This effect would lead to a misreconstruction of the tracks and increased noise behaviour. Another problem is the potential modification of the electric field, that could affect the charge drift. The MicroBooNE detector has recently seen an unexpected level of electronic noise; one of the suggested explanations was the movement of the wires in the electric field, likely induced 
by convection of liquid argon in the TPC [47]. Clearly, the higher the tension of the wire, the smaller this effect can be. The SBND detector specification is that wires must be tensioned at $4.9 \mathrm{~N}=500 \mathrm{~g}$, such that the sag is limited to $<0.5 \mathrm{~mm}[40]$.

It is crucial to measure the tension of the wires precisely to ensure proper detector operation. Recently constructed LArTPC detectors used a laser tensioning method described in Section 6.3. This and the following chapter present a novel, less invasive technique that exploits an electric circuit, that has been designed to be used during the construction and commissioning of the SBND detector.

\subsection{Generic principle of tension measurements}

Most tension measurement rely on the fact that it is possible to extract the tension $T$ of a wire from its resonant frequency. These two physical quantities are related by the equation

$$
T=4 L^{2} m f_{r}^{2}
$$

where

- $m$ is the wire mass per unit length;

- $L$ is the wire length;

- $f_{r}$ is the resonant frequency.

The problem to measure the tension becomes therefore a problem of measuring the fundamental resonant frequency of the wire. Equation 6.1 also shows that for a given tension the frequency will be a function of the wire length, which is illustrated in Figure 6.1 (at $T=4.9 \mathrm{~N})$.

SBND will use $150 \mu \mathrm{m}$ diameter Copper-Beryllium (CuBe) wires [24]. Knowing the density of $\mathrm{CuBe}$ allows to calculate the value of the tension once the frequency is known for all lengths of wires. This becomes slightly more complicated for the SBND detector because wires will be divided in segments by crossbars (see Figure 5.4). This results in a reduction of the effective length. The distribution of segment lengths after applying the combs is shown in Figure 6.2. As most wire segments will be on the order of $1.4 \mathrm{~m}$, we expect that most of the wires will have their fundamental frequency at a value close to $\sim 100 \mathrm{~Hz}$. 


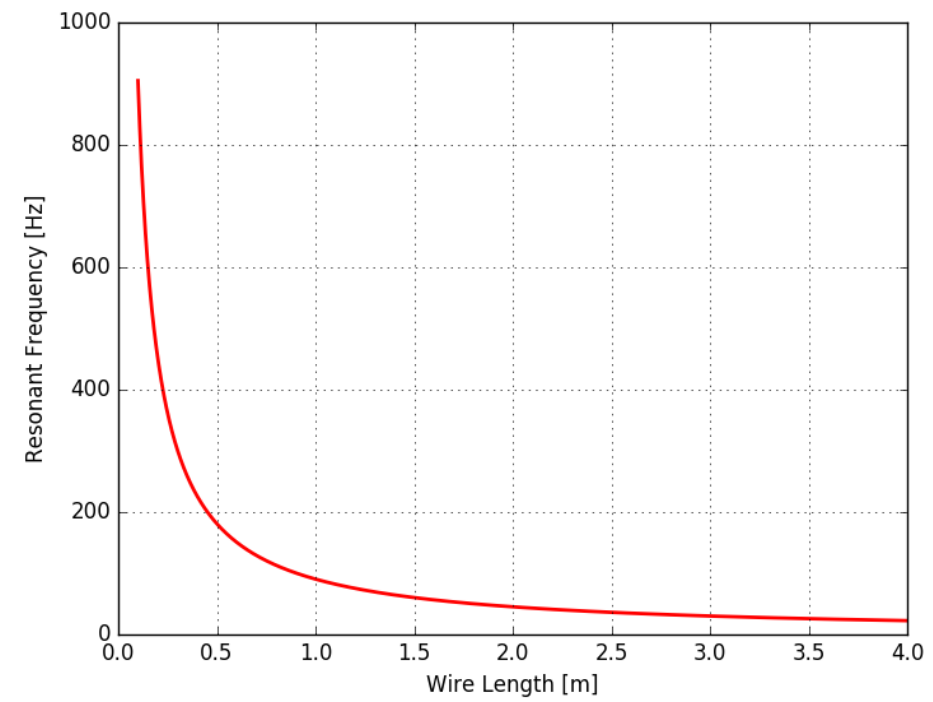

Figure 6.1: Frequency as a function of the wire length at $T=4.9 \mathrm{~N}$ for $150 \mu \mathrm{m}$ diameter Copper-Beryllium $(\mathrm{CuBe})$ wires.

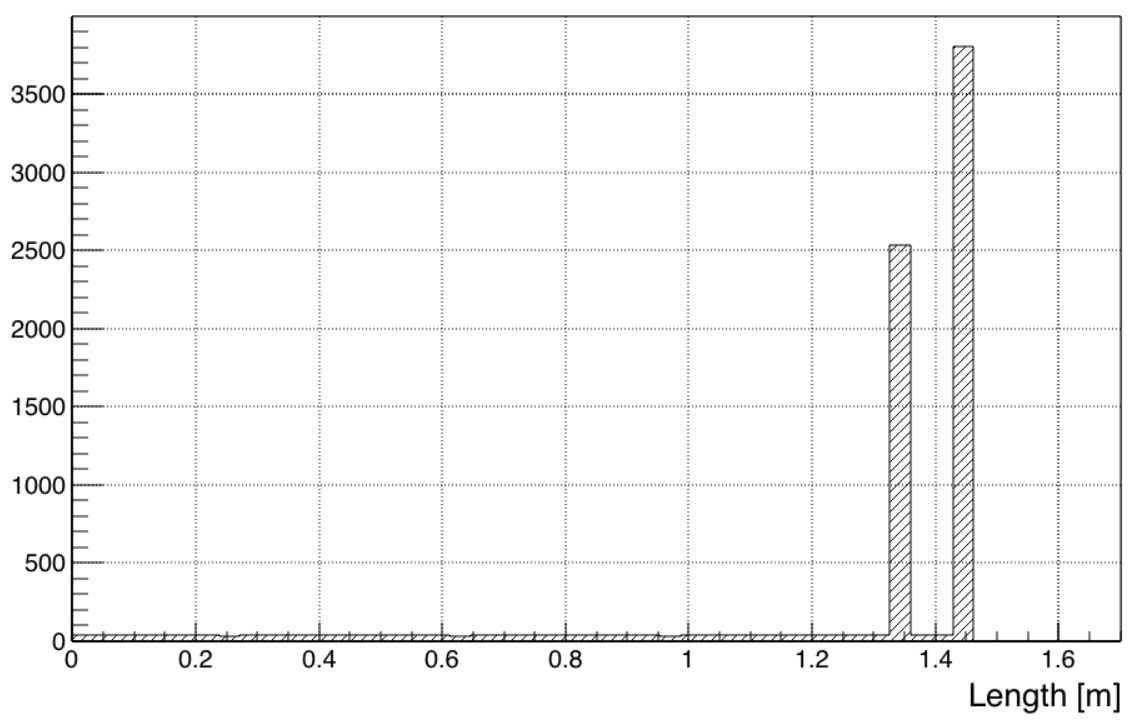

Figure 6.2: Distribution of wire lengths in the SBND detector after accounting for combs on crossbars. 


\subsection{Laser technique}

Until recently, the technique used to measure the wire tension in LArTPCs employed a laser-head and a photodiode [48]. The technique consists of focusing a laser on the wire to be measured and collecting the reflected light with the photodiode. A vibration is then externally induced on the wire so that the photodiode records the reflected light at the same frequency at which the wire is oscillating, which is the resonant frequency of the wire itself. The electric signal generated by the photodiode is then processed by software which performs a fast fourier transform (FFT) analysis of the signal, resulting in a frequency distribution. Once the resonant frequency is known, the tension $T$ can be obtained by means of Equation 6.1. Figure 6.3 shows an example of a laser-head device with integrated photodiode.

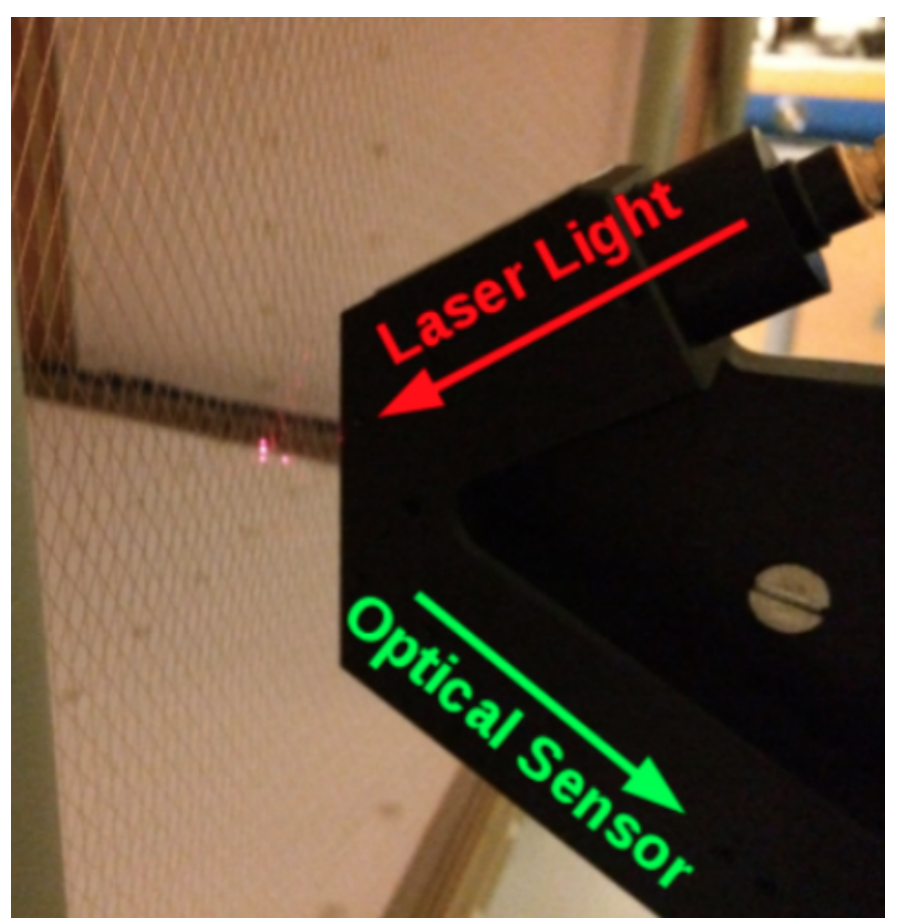

Figure 6.3: Dedicated laser tensioning device (taken from [48]). The laser light is reflected off of the wires and detected by the optical sensor.

The laser procedure is known to be precise and reliable but is somewhat inconvenient. The light needs to be focused on each single wire and the procedure may need to be repeated thousands of times in large detectors. In addition, given that wires are divided into two or more segments by combs, the laser procedure should in principle be applied to each segment, which could become tedious and time consuming. Furthermore, use of laser becomes very hard when we want to measure the tension of wires in the inner planes of the APA and impossible when the APAs are installed in the detector and the cryostat is closed. 
For these reasons there is an ongoing effort to develop a new, non-invasive, technique that could provide a more robust and quicker method of measuring wire tension in large scale LArTPCs. The rest of this chapter is devoted to the description of an electric method which resolves many of the inconveniences of the laser-head method.

\subsection{Electric technique}

It has been shown that an electric circuit exploiting a pair of adjacent wires can be used to measure the tension of both wires at the same time [49]. Figure 6.4 shows a scheme of the simplest circuit needed to perform such a measurement.

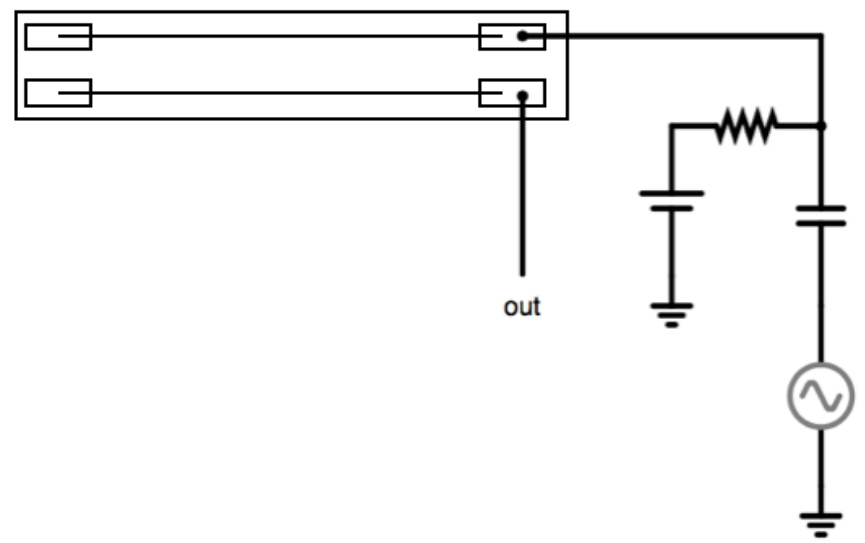

Figure 6.4: Basic circuit scheme for a wire tension measurement using an electric circuit.

One of the two wires is biased with a superposition of alternating and constant voltage, while the second is connected to a device to readout the induced signal. As a consequence, an electromagnetic force that is repulsive or attractive with the frequency of the AC signal is established between the two wires. The wire pair behaves like a capacitor with a capacitance changing with the frequency of the AC signal. Therefore, it is possible to read the induced signal on the un-biased wire by using an oscilloscope or a digitizer.

Assuming that the system behaves as a harmonic oscillator, the signal expected from the circuit in Figure 6.4 can be described by the following equation:

$$
V(t) \propto \frac{d q}{d t}=C_{0} v_{0} \omega \cos (\omega t)+C_{0} V_{0} a(\omega) \omega \cos (\omega t+\phi(\omega))+\mathcal{O}\left(v_{0} a\right),
$$

where

- $\omega$ is the angular frequency of the AC signal;

- $v_{0}$ is the amplitude of the AC signal; 
- $V_{0}$ is the value of the DC bias;

- $C_{0}$ is the capacity of the system composed by the two parallel wires without perturbation;

- $t$ is time;

- $a(\omega)=\frac{\omega_{r}^{2} a_{0}}{\sqrt{\left(\omega_{r}^{2}-\omega^{2}\right)^{2}+\beta^{2} \omega^{2}}}$ with $\beta$ being an unknown damping factor, $a_{0}$ a free parameter proportional to size of oscillation of the wires and $\omega_{r}$ the resonant frequency;

- $\phi=\arctan \left(\frac{2 \beta \omega}{\omega_{r}^{2}-\omega^{2}}\right)$.

An example behaviour of the signal as a function of the frequency of the alternating voltage is illustrated in Figure 6.5 which shows clear peaks at the values of the two resonant frequencies of the two wires being measured. The signal exhibits a linearly raising baseline (proportional to $\omega$ ) resulting from the first term of Equation 6.2.

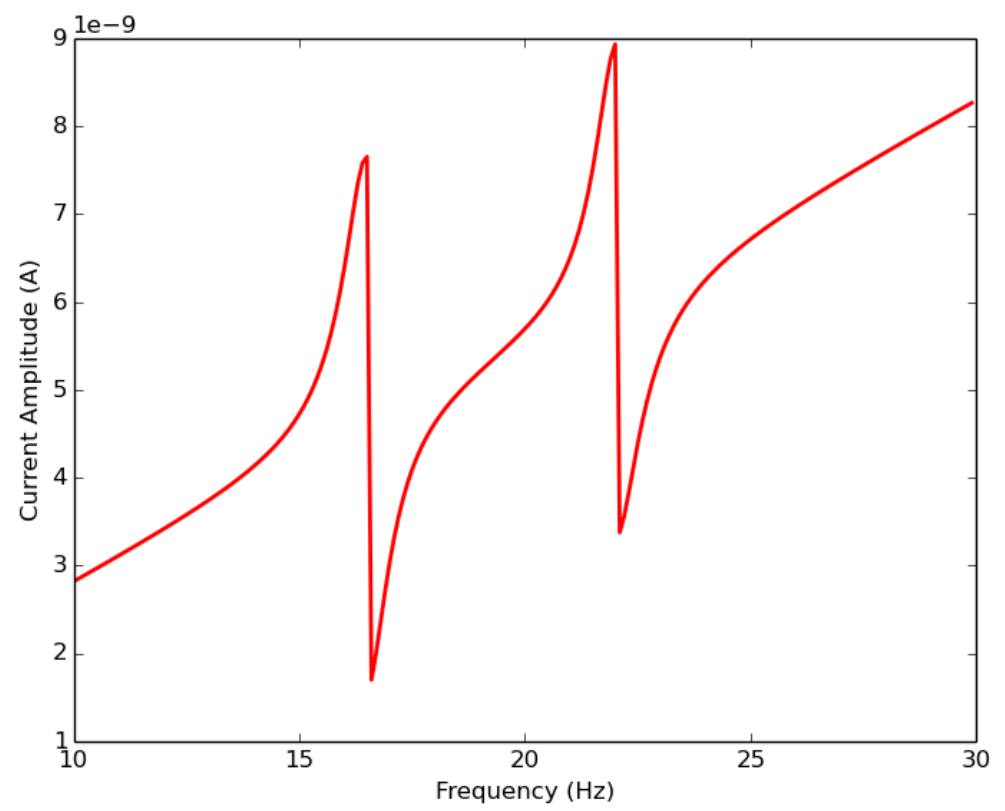

Figure 6.5: Expected signal with two resonant peaks. Values of parameters in Equation 6.2 are $\beta=0.9, v_{0}=8.7 \mathrm{~V}, V_{0}=1 \mathrm{kV}, a_{0}=3 \cdot 10^{-4}, \omega_{r 1}=16.5 \mathrm{~Hz}$ and $\omega_{r 2}=22 \mathrm{~Hz}$.

Therefore, a measurement involves a frequency scan of the alternating signal around the expected resonant frequency and identifying the resonant frequency from the peaks in amplitude. It is important to note that this technique allows simultaneous measurements of the tension of both wires thanks to the symmetry of the electric force acting on them, however with an inherent degeneracy because we do not know a priori which peak corresponds to which wire. 
This method presents several advantages compared to the laser method:

- there is no limit, in principle, on the number of wires that can be measured at the same time. The circuit scheme can be replicated for all the wires whose tension needs to be measured.

- as it is a non-invasive technique, i.e. there is no need to manually perturb the wires, it is possible to make measurements once the APAs are installed in the detector, provided the electronic boards are designed to allow for this. Furthermore, it has been shown (see Section 7.4) that the technique works even at cryogenic temperatures.

- if wires are divided into segments by means of combs, it is possible to measure the tension of each segment that make up the wire simultaneously.

- measuring the wire tension with the electric method ensures the electrical continuity of the wires is also tested.

\subsection{Fit model and frequency extrapolation}

A fit model was used to determine the resonant frequencies from data obtained in the measurements presented in this dissertation. A formula derived from Equation 6.2 was applied:

$$
F(\omega)=A_{1}\left(C_{0} v_{0} \omega-V_{0} C_{0} \omega a_{1}(\omega)\right)+A_{2}\left(C_{0} v_{0} \omega-V_{0} C_{0} \omega a_{2}(\omega)\right)
$$

where

$$
a_{i}(\omega)=A_{3} \frac{\omega_{0 i}^{2}-\omega^{2}}{\left(\omega_{0 i}^{2}-\omega^{2}\right)^{2}-(2 \Gamma \omega)^{2}}, \quad i \in\{1,2\} .
$$

The definitions of the symbols are the following:

- $A_{1}, A_{2}$ and $A_{3}$ (same for $i=1,2$ ) are overall constants, free parameters of the fit;

- $a_{i}(\omega)$ is the elastic amplitude for a driven damped harmonic motion: $\omega_{0 i}$ is the resonant frequency of the $i$-th peak and $\Gamma$ is an unknown damping factor. These parameters are also determined by the fit.

$v_{0}, V_{0}$ are the two fixed biasing voltages. $C_{0}$, the capacitance between the wires with no oscillation, is known given the length $l$ of the wires: $C_{0}=l \frac{\pi \epsilon}{\ln \left(\frac{d-r}{r}\right)}$ where $r$ is the wire radius, $d$ is the distance between the wires and $\epsilon \equiv \epsilon_{0}=8.854 \mathrm{~F} / \mathrm{m}$.

The algorithm scans the data and determines if there are one or two resonant peaks. In the former case the parameter $A_{2}$ is fixed to zero. An example of a fit with two resonant 


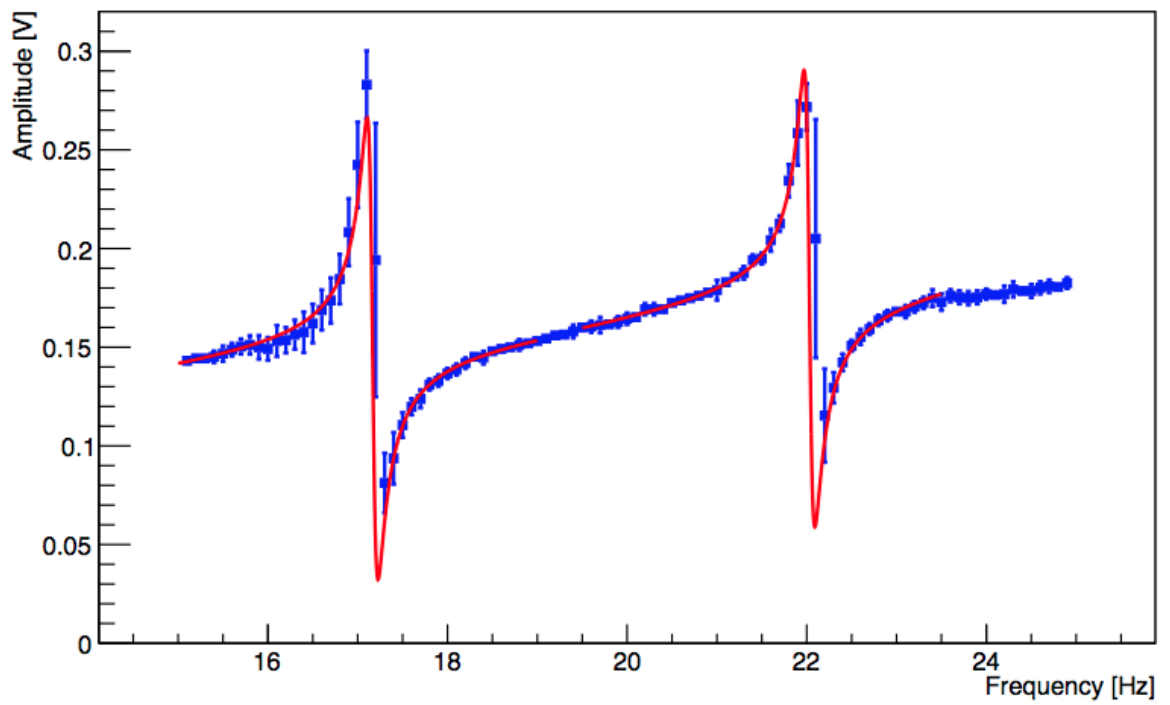

Figure 6.6: Example of the signal of two wires at different tensions with the fit. The measurement was taken with the long-frame set-up, circuit $V 1$ (described in the next chapter). The estimated resonant frequencies are $17.16 \mathrm{~Hz}$ and $22.03 \mathrm{~Hz}$.

peaks is shown in Figure 6.6: the estimated resonant frequencies are $17.16 \mathrm{~Hz}$ and $22.03 \mathrm{~Hz}$. This method was applied throughout the measurements, described in the next chapter, to estimate consistently the resonant frequencies. 


\section{Chapter 7}

\section{Developing the Tension}

\section{Measurement Method for SBND}

In this chapter we describe the experimental components used during the development of the electric tension measurement described in this thesis. A chronological description of the development of the set-up will be presented. The application of the method to LArTPC experiments with particular regard for the SBND detector will be also discussed.

\subsection{Electronic circuits and wire frame set-ups}

Different wire mounting set-ups (frames) and electric circuits have been developed and used throughout the year in order to improve the tension measurement method. This section is dedicated to their description and can be used as a reference through this chapter.

\subsubsection{Wire-frame set-ups}

Developing the technique required looking at different lengths of wires and so we used three different configurations which we will call: long-frame, short-frame and SBND test-frame. In all cases, the frames provided a structure on which the wires were strung and affixed to PCB boards at either end. What follows is a description of the different set-ups used during the tests described in this chapter.

\section{Long-frame $(4 \mathrm{~m})$}

The length has been chosen to match the longest wires in the SBND APAs if no combs are used. The wires were soldered on the two edges on PCB boards. Figure 7.1 shows a photo of the frame. 


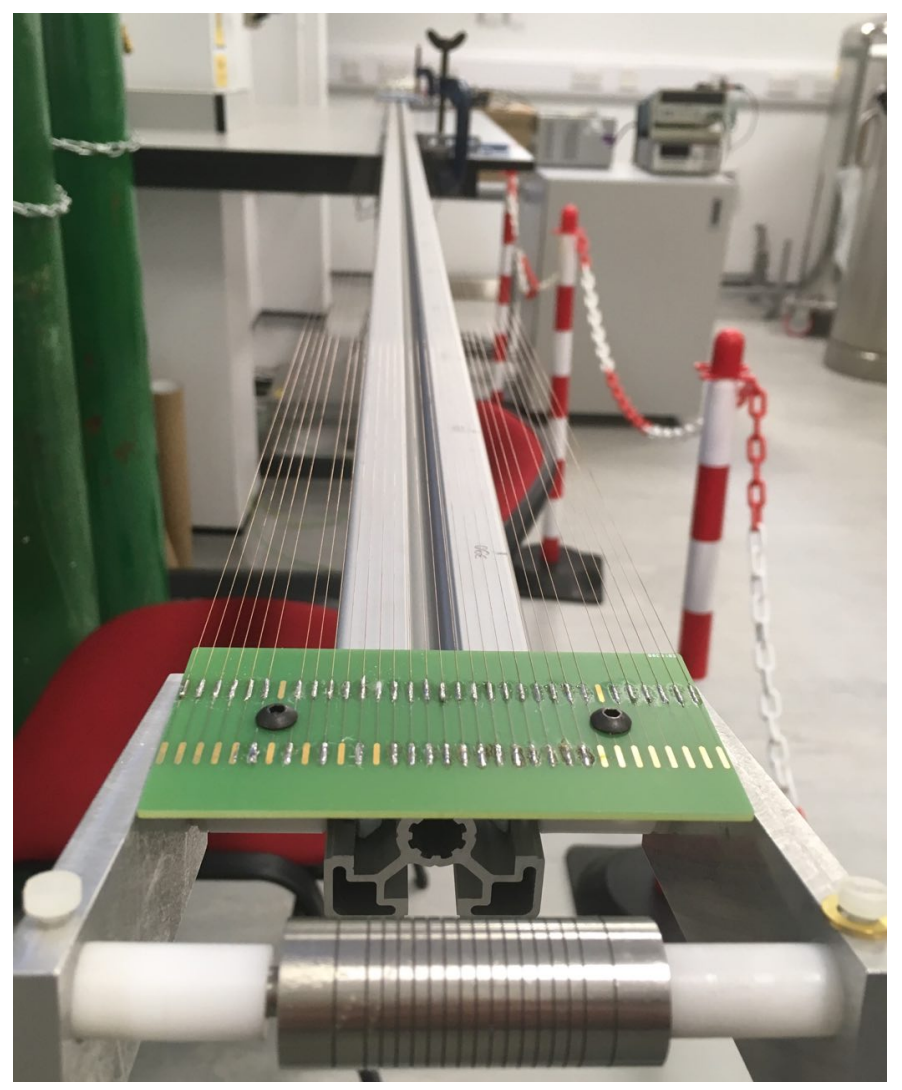

Figure 7.1: $4 \mathrm{~m}$ long frame with soldered wires.

This arrangement allows to test the circuits and the technique with wires of different length by means of moveable combs, such as those shown in Figure 7.2.

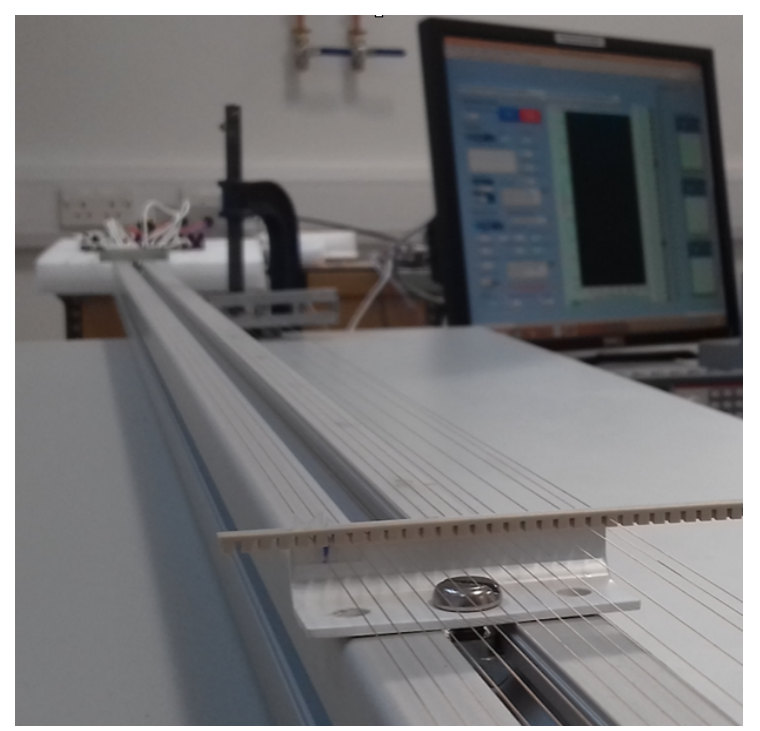

Figure 7.2: Movable combs on the $4 \mathrm{~m}$ frame. These combs allowed clamping down on the wires and dividing the wires into well defined segments. 


\section{Short-frame $(72 \mathrm{~cm})$}

A second set-up that was prepared consisted of a short-frame with twenty-six $72 \mathrm{~cm}$ long wires. The purpose of this set-up was to test different ways of attaching the wires to an electronic board. The length of the frame (see Figure 7.3) is also short enough to allow for a cold test in a dewar present in our lab (see Section 7.4). To understand the behaviour of the set-up in cold, the frame and the boards were chosen to be of the same identical material of the real APAs.

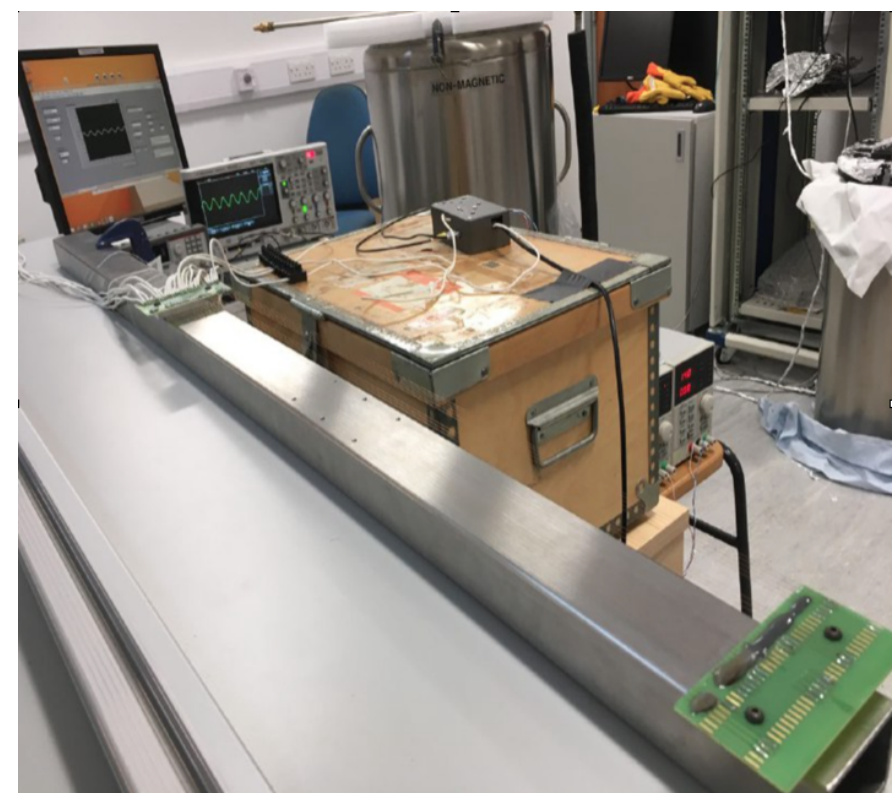

Figure 7.3: Photo of the short-frame. Different methods of wires attachment are visible on the right.

\section{SBND test-frame}

This set-up is a mock-up of an SBND APA assembly frame. It has wires at lengths of $133 \mathrm{~cm}$. It was used to test the electric tension method with real SBND electronic boards. A photo of the test-frame is visible in Figure 7.4.

\subsubsection{Wire soldering and tension preparation}

Wires were soldered on electronic boards that were firmly fixed to the stainless-steel frames. Apart from the SBND test-frame at Daresbury, the procedure was manual: an edge of the wire was soldered on the holding-signal board, then the wire was pulled along the frame and a weight was fixed to the other end. By means of a pulley, the weight was left hanging keeping the wire in tension (see Figure 7.5). While kept in tension, the wire was soldered on 


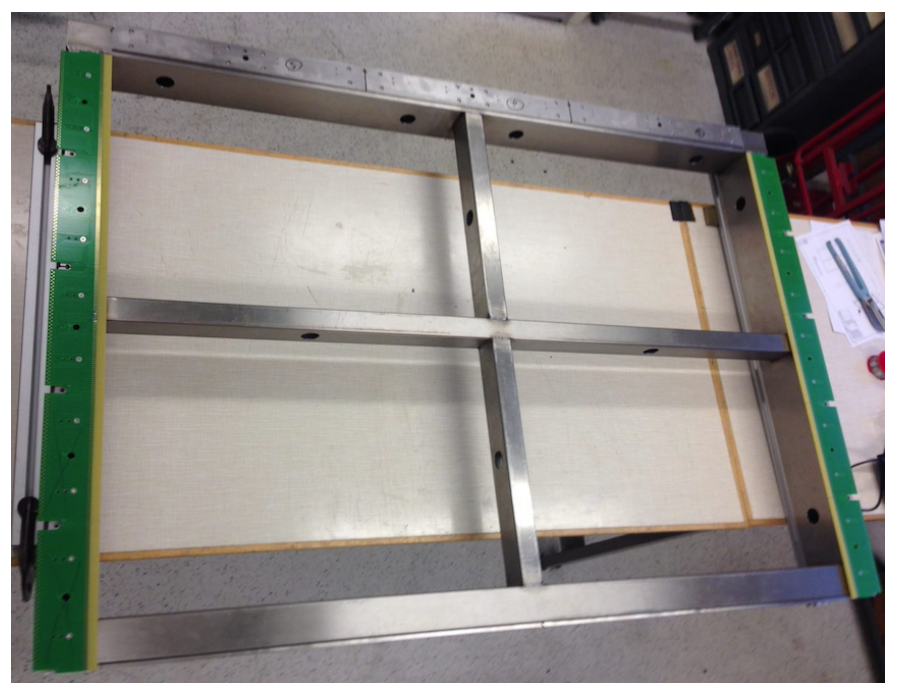

Figure 7.4: Test-frame at Daresbury Laboratory. Geometry boards are present on the left and right.

the far electric board, not connected to the readout electronics.

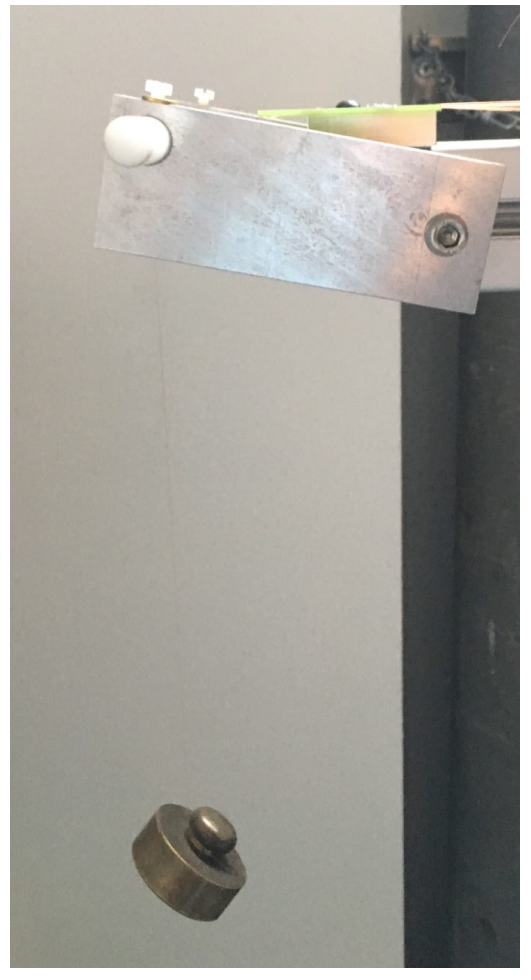

Figure 7.5: Photo of weight used in the wire tensioning procedure.

In the case of the frame at Daresbury, a robot head was used to prepare and tension the wires by a group of engineers and technicians. 


\subsubsection{SBND boards and limitations}

SBND U \& Y wire planes are biased at different values [24], therefore capacitors and resistors are needed to protect the readout channels from the high voltages. See Figure 7.6 for a drawing of the connections [50].

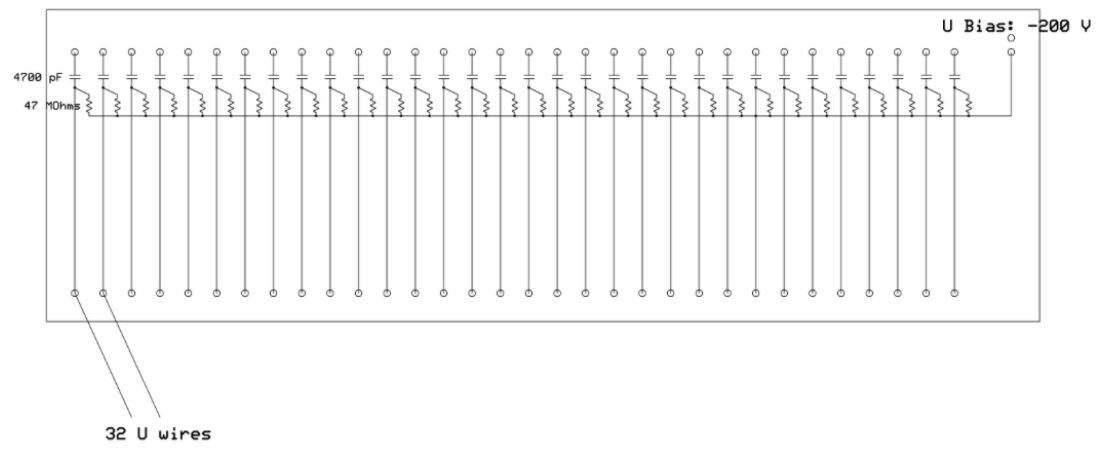

Figure 7.6: Drawing of a U-TOP geometry board circuit [50].

The value of the resistors on every board is $47 \mathrm{M} \Omega$ and the capacitors are $2.2 \mathrm{nF}$ or $4.7 \mathrm{nF}$. Resistors have a nominal tolerance of $200 \mathrm{~V}$. If we were to connect two adjacent wires (connection in the bottom of Figure 7.6) to the circuit, then the maximum value of the voltage that can be applied is $400 \mathrm{~V}$, understood as the sum of the constant bias and the amplitude of the AC signal: $\left(v_{0}+V_{0}\right)$. In this configuration the maximum value across each resistor would not be greater than the maximum tolerance, as the circuit would be closed by two resistors in series. This requirement has guided the need to reduce the voltage in our set-up.

\subsubsection{Electronic circuits}

The readout of the signal as well as the biasing of the wires was done by cables soldered to the paths on the holding-signal board. These cables were then connected to the custom electronic circuits to bias and readout the signals. Figure 7.7 shows one board with the copper-beryllium and soldered readout wires. The PCB electronic boards used in our lab had two electrically connected copper slots for each wire: one to allocate the wire and one for the readout cable. The pitch between the slots was $3 \mathrm{~mm}$, the same as the SBND APA design [40].

As we were developing the method we used three different versions of the electronic circuit. These will be called $V 1, V 2$ and $V 3$ :

- $V 1$, a basic implementation of the circuit of Figure 6.4 with an "instrumentation amplifier" added: the component was a useful add-on to the standard circuit: it allowed 


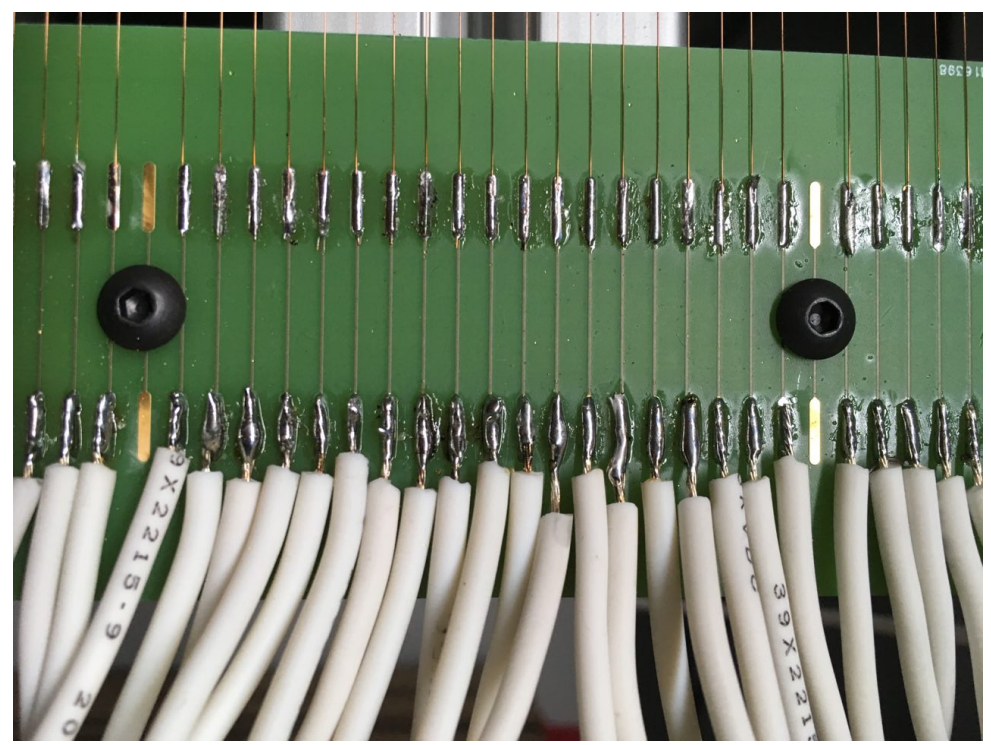

Figure 7.7: Copper-beryllium wires (top) and readout cables (bottom) soldered on the holding-signal PCB board.

to significantly reduce the noise in the signal exploiting a spare wire as an antenna to catch the noise and subtract it from the signal before it got to the oscilloscope or digitizer (see discussion in Section 7.2.1).

- $V 2$, an upgrade designed to reduce the voltages needed to operate the system. The wires surrounding the wire to measure are biased. This system also removes the degeneracy between the two wires measured in $V 1$.

- $V 3$, an expansion of $V 2$ in order to measure 32 wires at the same time.

In every case, a standard signal generator with USB interface (Keithley 3390 model) was used to provide the AC signal. Since it can provide a signal with a maximum amplitude of $10 \mathrm{~V}$, whenever a larger amplitude was needed a linear voltage amplifier with its power supply ( $24-30 \mathrm{~V}$ DC) was employed. Also, low voltage supplies ( $\pm 12 \mathrm{~V} \mathrm{DC})$ were used to power the "instrumentation amplifier".

\section{Readout}

In most cases, the read out wires and circuit were connected by a screw connector. In the case of the SBND test-frame, the circuit was connected to the wires using a specifically designed connector to be fixed directly on an SBND readout electrical board. It had pins on the top to connect the wires coming from the circuit and pins between two metal slabs to sandwich the electronic board mounted on the frame (see Figure 7.8). 


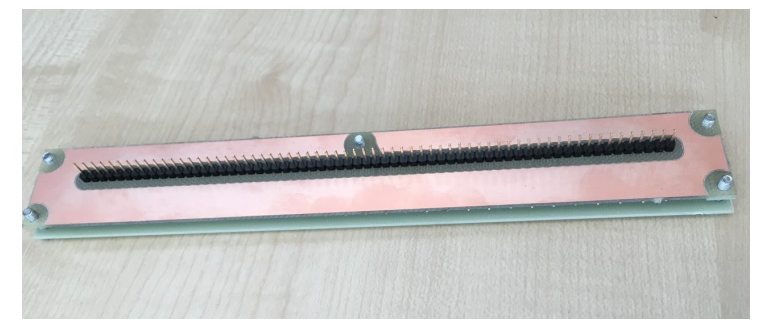

Figure 7.8: Board connector.

Early measurements of the circuit output amplitude were taken with an oscilloscope (Agilent DSO-X 2024A). It then was replaced by a CAEN DT5740 Digitizer, an analogto-digital converter able to readout up to 32 channels at the same time. A PC running LabVIEW acquisition software was employed to control the AC signal generator and to store the measurement from either the oscilloscope or the digitizer. The PC was connected to the devices via a standard USB interface.

\subsubsection{DAQ}

The data acquisition was performed using a LabVIEW script and underwent a natural evolution together with the hardware set-ups and the electronic circuits. Its purpose was to set the range in frequency and the amplitude of the AC signal generator and to store the amplitude of the output signal collected by the oscilloscope or the digitizer. Among the different versions, common features included the possibility to set the lower and upper extreme for the frequency range and the step width of the scan. The step width was kept fixed at $0.1 \mathrm{~Hz}$ for most of the measurements in this chapter, but it was a useful parameter that could be used to change the speed of the acquisition to carry out tests. A screen-shot of the final version of the script, realised for the version $V 3$ of the circuit and the CAEN digitizer, is shown in Figure 7.9. The parameters are set on the left-hand side of the screen. Among the parameters, the most important are:

- number of iterations used by the script to calculate the amplitude of the signal. A higher number implies a more precise but slower measurement.

- channel length. It must be of the form $3 \cdot 2^{N}$. A higher number means a wider acquisition window in terms of time interval, i.e. a larger fraction/number of the wavelength/period is sampled, respectively. Since the wire resonant frequency depends on the length, this number had to be adjusted when measuring wire of different length.

- amplitude of the signal coming from the signal generator. 
- value of the frequency step in the scan.

- the path for the ASCII files containing the value of the amplitudes and their dispersion.

The central window displays the sampled signal of the selected channel(s) with a maximum of 32 . The section on the right-hand side displays any errors, which is useful for debugging purposes.

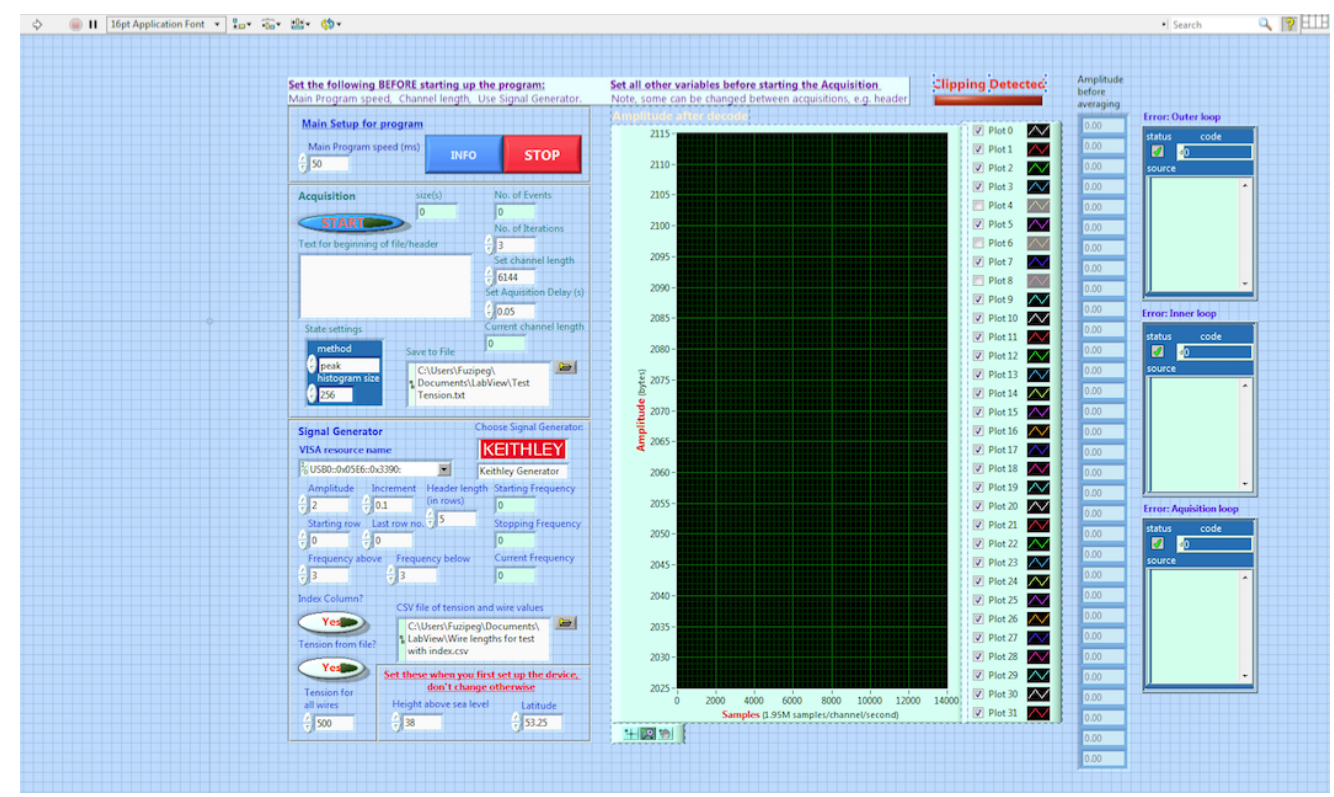

Figure 7.9: A screen-shot of the LabVIEW acquisition script.

The DAQ saved an ASCII file ("LVM file") that stores N columns in the format

$$
\mid \text { Frequency }|\mathrm{A}(1)| \operatorname{RMS}(1)|\ldots| \mathrm{A}(\mathrm{N})|\operatorname{RMS}(\mathrm{N})| \text {, }
$$

where $A(n)$ is the amplitude of the signal in channel $n$ at the given frequency and $\mathrm{N}$ is the number of channels that the current version of the circuit was able to readout. The RMS value is worked out as the dispersion of the measurements around their mean value.

The final version of the DAQ can read an external input file to automatically determine the range of frequencies to scan. This file is another ASCII file (CSV file) which contains information about the nominal tension of the wires and the length of each of the segments that make up the wires. The script reads the file and calculates the range to scan to include the resonant frequency of each segments. It has up to 4 columns: the first one contains the assumed tension of the wire in grams. The other three columns contain the length of the segments in which the wires is divided by combs. Some of the last three columns can be empty but commas, ",", must be present. As an example of the structure see Table 7.1. The script was designed having in mind the realistic situations for which it is necessary to 
measure the tension of a large number of wires. In that case it would be necessary to create an input file containing the information on each wire in the set-up in order to speed up the measurement.

Table 7.1: Structure of the input file for DAQ script. An " $\mathrm{x}$ " symbol means that the field is left empty.

\begin{tabular}{cccc}
\hline Expected tension [g] & Length seg. 1 [m] & Length seg. 2 [m] & Length seg. 3 [m] \\
\hline \hline 500 & 1.5 & $\mathrm{x}$ & $\mathrm{x}$ \\
500 & 1.5 & 1.5 & $\mathrm{x}$ \\
480 & 1.25 & $\mathrm{x}$ & $\mathrm{x}$ \\
460 & 1.3 & 1.5 & 1.3 \\
$\ldots$ & $\ldots$ & $\ldots$ & $\ldots$ \\
$\ldots$ & $\ldots$ & $\ldots$ & $\ldots$ \\
\hline
\end{tabular}

\subsection{Evolution of the circuit and method}

\subsubsection{Early measurements and noise reduction}

At first iteration, the circuit in Figure 6.4 was built and installed in an interface box. The interface box prevented accidental contact with the part of the circuit biased at high voltage and provided the slots to connect the cables for the high voltage supply and the AC signal generator. Early measurements (Figure 7.10) were taken with two adjacent wires tensioned at two different values. In this plot the value of the DC bias was $1 \mathrm{kV}$ and the $\mathrm{AC}$ amplitude was $10 \mathrm{~V}$. The plot shows two clear peaks at around $16 \mathrm{~Hz}$ and $22 \mathrm{~Hz}$ corresponding to a tension of $242 \mathrm{~g}$ and $457 \mathrm{~g}$. One of the first concerns was the dip observed at $25 \mathrm{~Hz}$. To understand this, a scan of the noise in a larger frequency range led to the data plotted in Figure 7.11. To only see the noise, the DC bias was not switched on, resulting in the absence of the two peaks, leaving only the linear baseline.

Other notable features were observed: large noise around $50 \mathrm{~Hz}$ and $25 \mathrm{~Hz}$. Given the values, this behaviour was ascribed to electromagnetic noise coming from the mains. As this noise could hide or distort the resonant signal should it happen to be in the vicinity of these frequencies, an attempt was made to reduce it.

The method used to reduce the noise was to use a so-called "instrumentation amplifier". This device uses a third wire, not exploited by the circuit, as an "antenna" to catch the noise and subtract it from the output signal of the circuit. The first amplifier developed is shown in Figure 7.12: the white cable in the picture had to be connected to the antenna via the screw connector and there are two slots, one for the output signal of the circuit to get in 


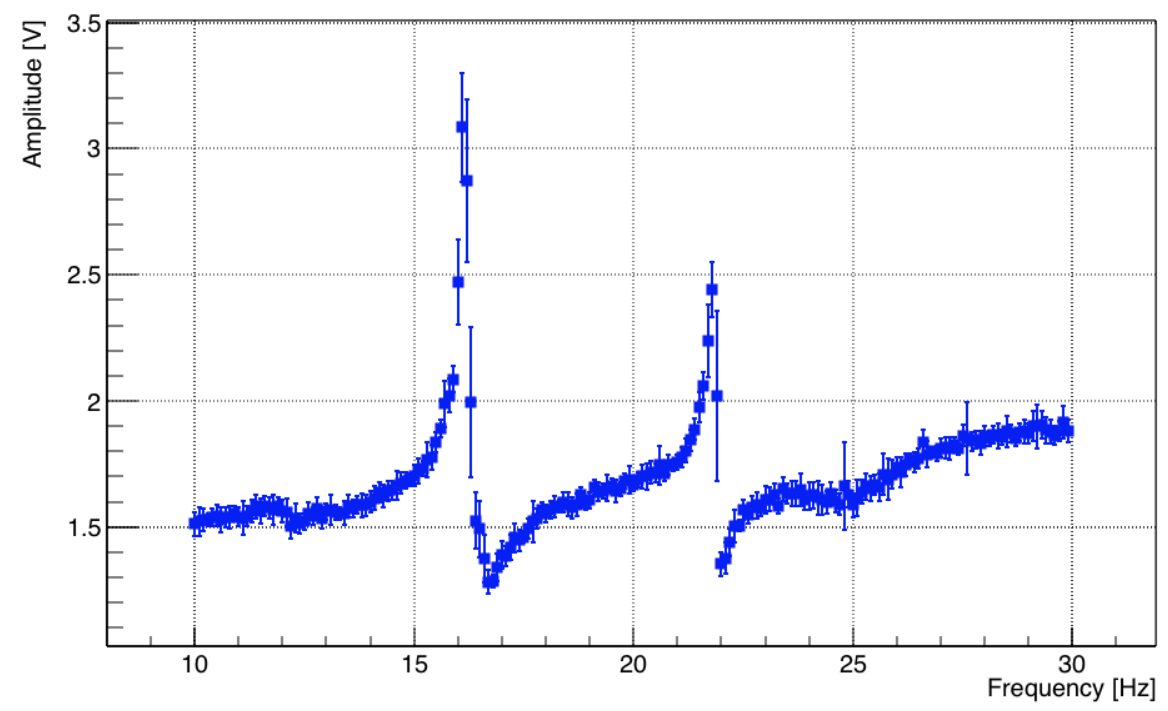

Figure 7.10: Signal for wires soldered at two different tensions with constant bias of $1 \mathrm{kV}$. The measurement was taken with $V 1$ and the long-frame.

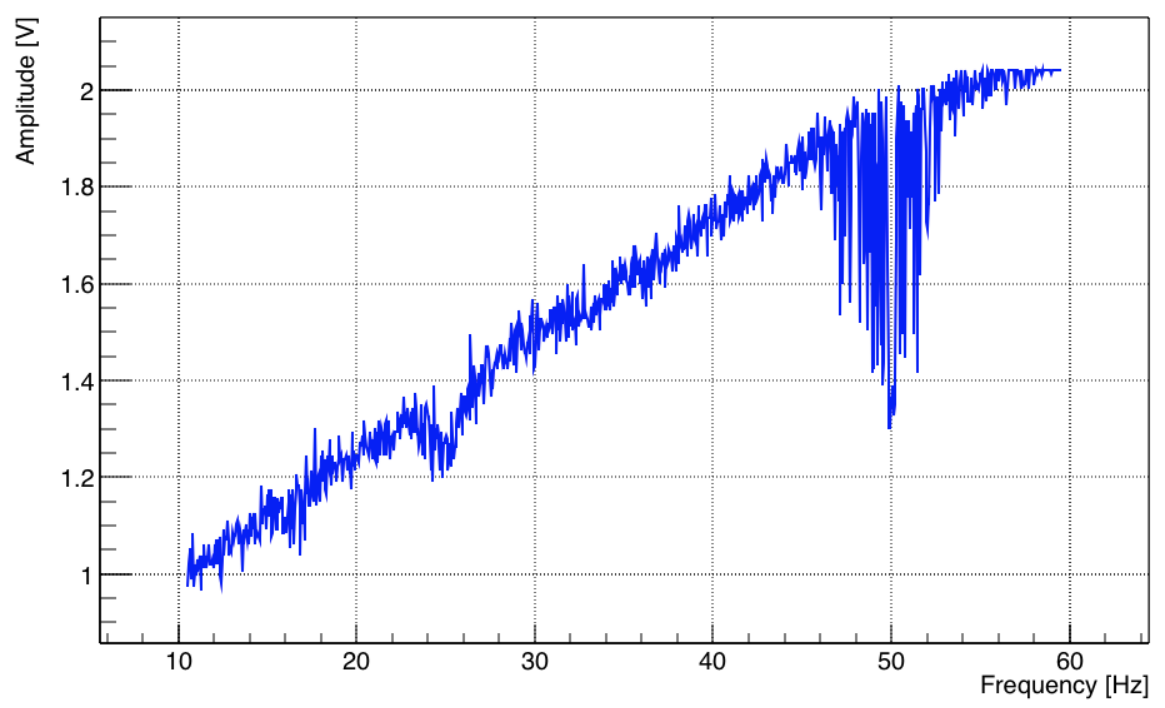

Figure 7.11: Signal without high voltage. The measurement was taken with $V 1$ and the long-frame. 
and the other for the "cleaned" signal to get out. The circuit needs to be powered and this is done via another external power supply. By means of a screw (on the top of the blue box in Figure 7.12) it is possible to manually adjust the response of the amplifier.

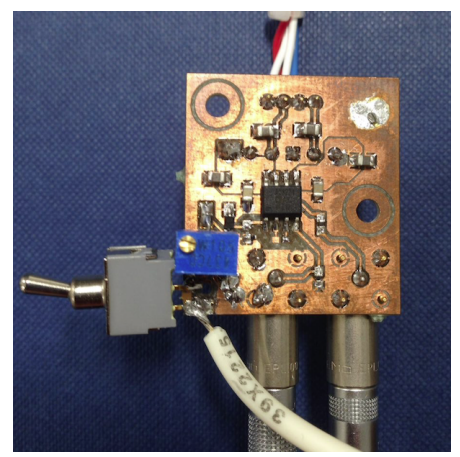

Figure 7.12: Instrumentation amplifier. See text for description.

Figure 7.13 shows the baseline of the same signal with (lower curve) and without (upper curve) the instrumentation amplifier. The improvement is clearly visible, especially around

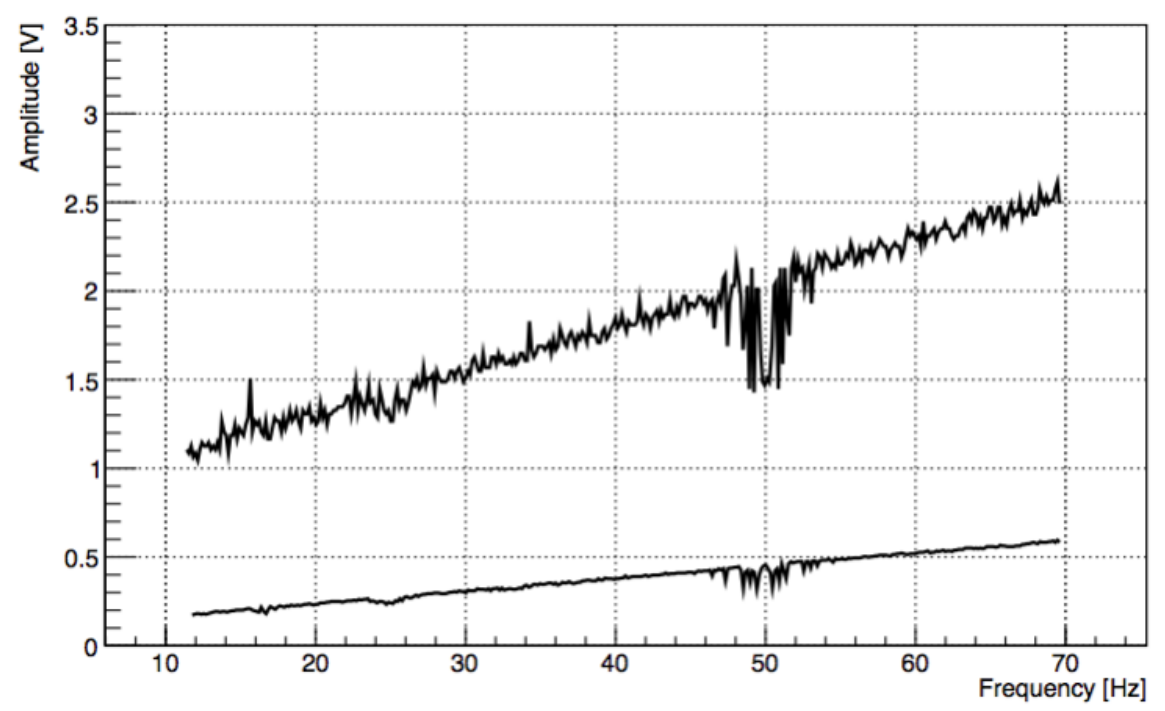

Figure 7.13: Noise before (higher points) and after (lower points) the addition of the instrumentation amplifier. The circuit employed was $V 1$ together with the long-frame.

$50 \mathrm{~Hz}$. Thanks to this addition, it was possible to reduce the value of the constant bias to $500 \mathrm{~V}$ maintaining clear signals. Figure 7.14 shows the same configuration as Figure 7.10 but with a $500 \mathrm{~V}$ constant bias after the applying of the instrumentation amplifier.

\subsubsection{Upgrade of the circuit to use three wires}

A new circuit idea, $V 2$, was developed with the aim of reducing the values of the biasing voltages. The new concept consists of biasing the two wires surrounding the wire whose 


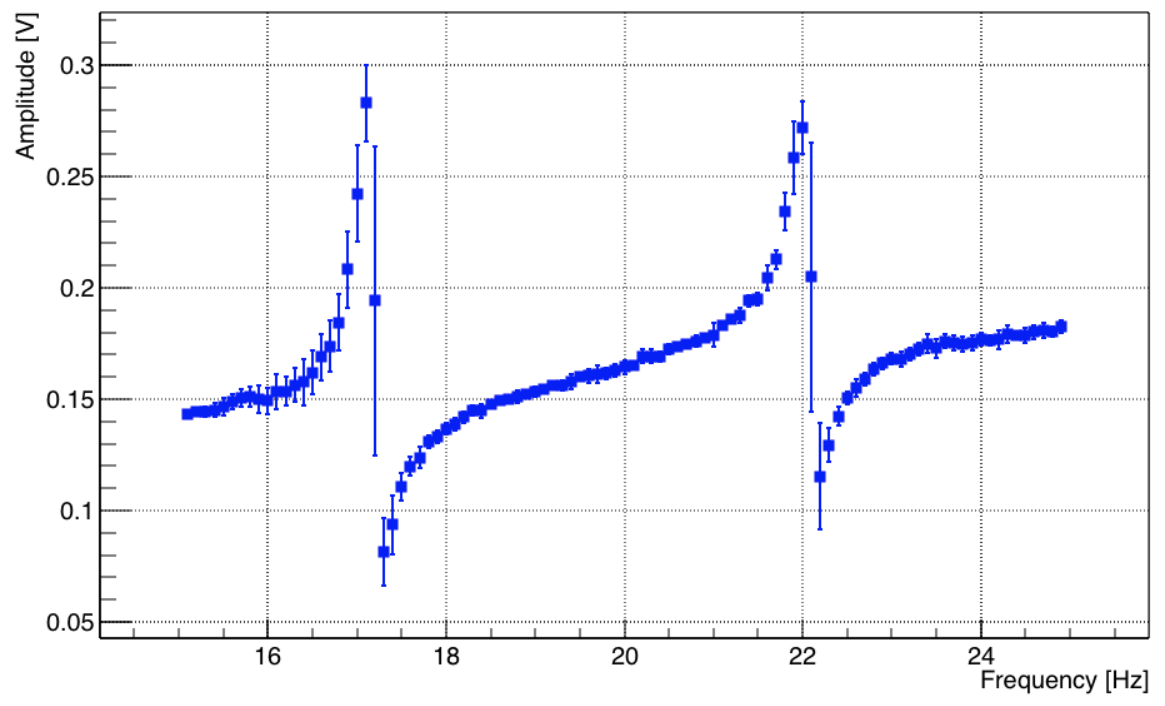

Figure 7.14: Signal for wires soldered at $300 \mathrm{~g}$ and $500 \mathrm{~g}$ with a constant bias of $500 \mathrm{~V}$. The circuit employed was $V 1$ with the instrumentation amplifier together with the long-frame.

tension is being measured by an $\mathrm{AC}$ with added or subtracted a $\mathrm{DC}$ voltage $\left(v_{0} \pm V_{0}\right)$ on opposite sides. This new configuration allows the use of smaller values of the voltages that would not damage the $R C$ components of the SBND electronic boards. In this case, due to the fact that two different constant biases must be applied, $\pm V_{0}$, the limit to preserve the SBND boards (see Figure 7.6) is lowered to $\pm\left(v_{0}+V_{0}\right)= \pm 200$ V. Figure 7.15 shows a cartoon explaining the idea. The red dashed lines represent the wire whose tension we want to measure, while the black ones are the biased wires. The oscillation is enhanced by the opposite electric fields on each side of the grounded wires. This structure is also easy to replicate for a larger number of wires. A schematic drawing of the new circuit is shown in Figure 7.16. The left-hand part shows the circuit used to bias the wires wile the right-hand part shows the instrumentation amplifier together with the digitizer and the laptop used for DAQ.

The new configuration increased the amplitude of the signals by a factor of five with respect to the signal obtained with the $V 1$ configuration. Another advantage is that it resolves the ambiguity that we would have between the two adjacent wires used before: the new set-up measures the resonant frequency of three wires, but only the signal corresponding to the wire in the middle is enhanced. This mechanism thus allows easy identification of the frequency of the wire for which the tension is measured. 


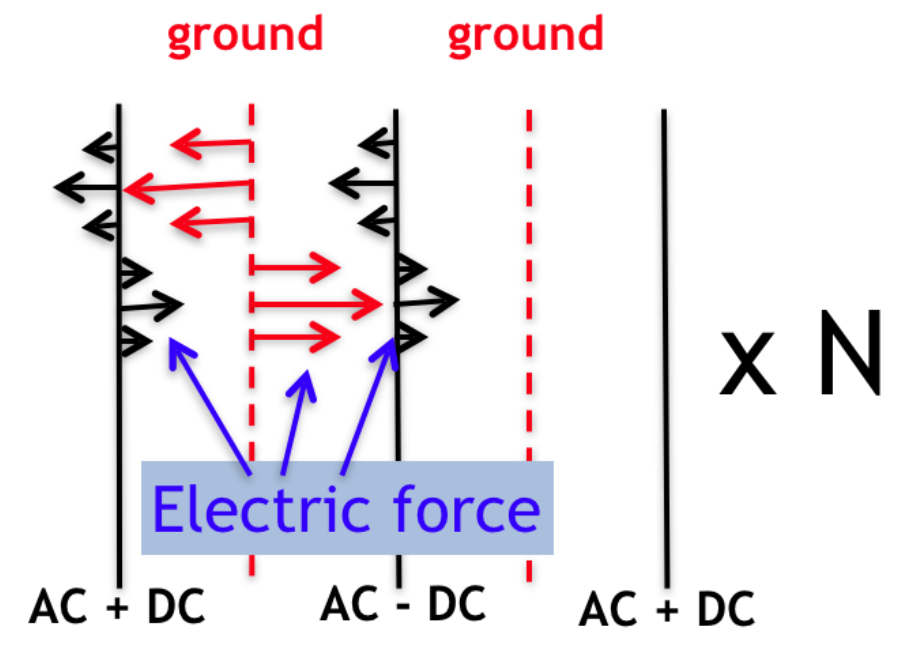

Figure 7.15: Cartoon explaining the method of operation of the $V 2$ version of the circuit

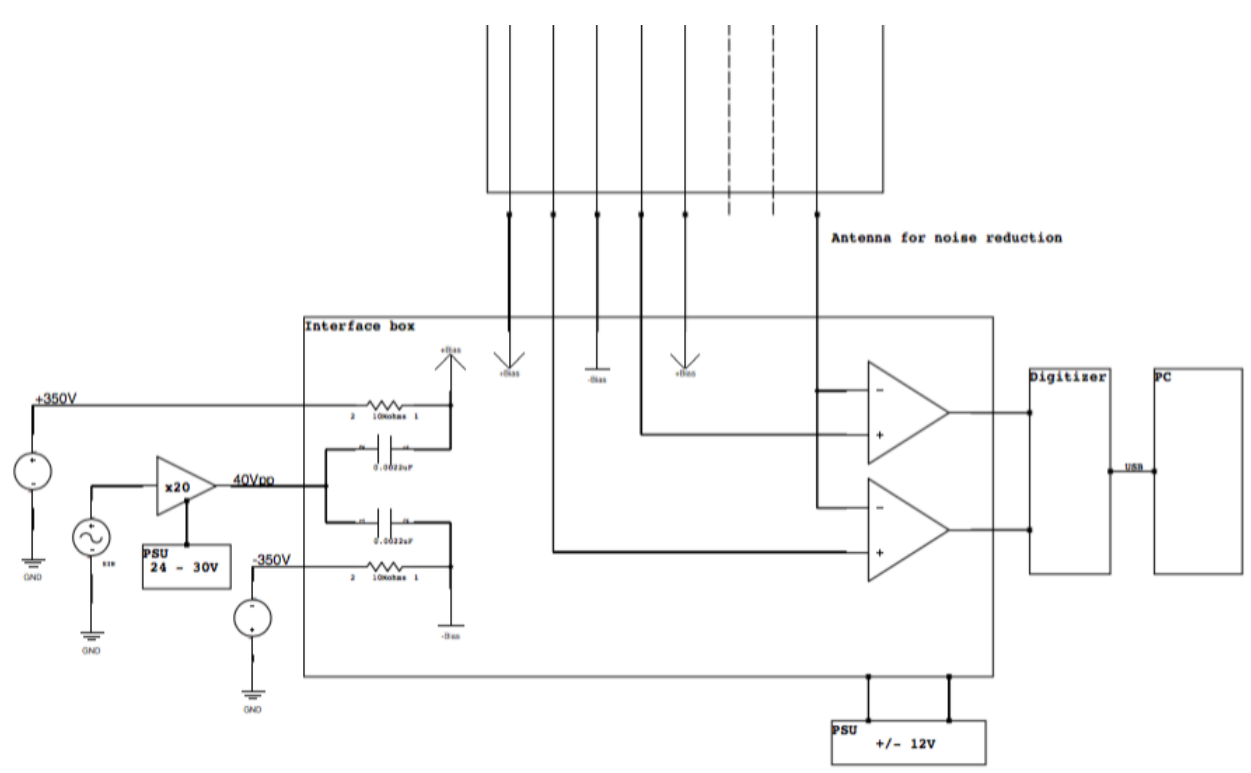

Figure 7.16: Electric scheme of the $V 2$ circuit. 


\section{Benchmarking the $V 2$ circuit}

A systematic study was done to benchmark the $V 2$ circuit. The objective was to characterise the length of the wire we could measure staying below the SBND board limit. The measurements were taken exploiting the $4 \mathrm{~m}$ long frame and using combs to investigate the behaviour at various lengths in the SBND regime $(\lesssim 1.5 \mathrm{~m})$ (see Figure 6.2$)$. After a dedicated survey, the optimal value found for the AC signal amplitude was $v_{0}=40 \mathrm{~V}^{1}$ this allows for a strong signal, while avoiding saturation of the amplifiers. It was kept for subsequent measurements. To characterise the strength of the signal for different lengths, measurements at $1.5 \mathrm{~m}, 1 \mathrm{~m}$ and $0.75 \mathrm{~m}$ were taken. For each of them, two values of the constant bias $V_{0}$ were used:

- $V_{0}=150 \mathrm{~V}$, to stay below the SBND limit: $V_{0}+v_{0}=150 \mathrm{~V}+40 \mathrm{~V}=190 \mathrm{~V}<200 \mathrm{~V}$;

- $V_{0}=350 \mathrm{~V}$, resulting in more pronounced signals to compare with the SBND limit conditions.

To quantify the strength of the signal, i.e. how is the resonant pattern distinguishable from the baseline, a signal-to-noise ratio $\frac{S}{N}$ parameter was defined as the ratio between the amplitude peak-to-peak of the resonant pattern and the average noise of the single amplitude measurement.

Figure 7.17 shows data for $1.5 \mathrm{~m}$ long wires: the left panel shows data taken with constant bias $V_{0}=350 \mathrm{~V}$, while the right panel shows measurements with $V_{0}=150 \mathrm{~V}$. Both signals

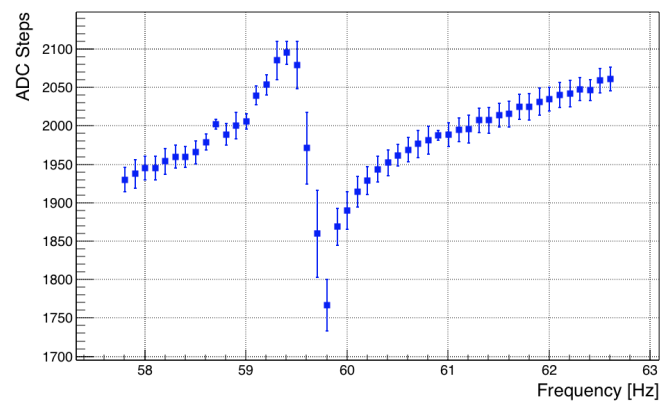

(a) $1.5 \mathrm{~m}$ long wires at $V_{0}=350 \mathrm{~V}, \frac{S}{N}=17.55$.

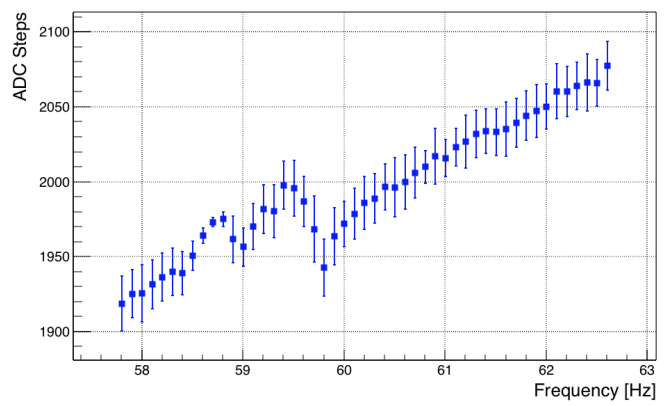

(b) $1.5 \mathrm{~m}$ long wires at $V_{0}=150 \mathrm{~V}, \frac{S}{N}=3.51$

Figure 7.17: Comparison between beyond and below the SBND voltage limit for $1.5 \mathrm{~m}$ long segments. Note the presence of bigger peak in correspondence of the resonant frequency of the wire being measured. See text for details.

have a bigger peak at the resonant frequency of the sandwiched wire and a small bump on the left corresponding to the resonant frequency of one of the two adjacent wires. Although the signal in Figure 7.17(a) is more clear, the resonant peak in Figure 7.17(b) is still clearly

\footnotetext{
${ }^{1}$ The linear amplifier was used to get to that value.
} 
visible. This would not have been possible without the noise reduction system and with the old version of the circuit (the oscillation amplitude would not have been large enough).

Figure 7.18 shows the same plots for $1 \mathrm{~m}$ long wires. In this case, both the two small peaks relative to the two adjacent wires are visible and, again, the main resonant peak is visible in both conditions.

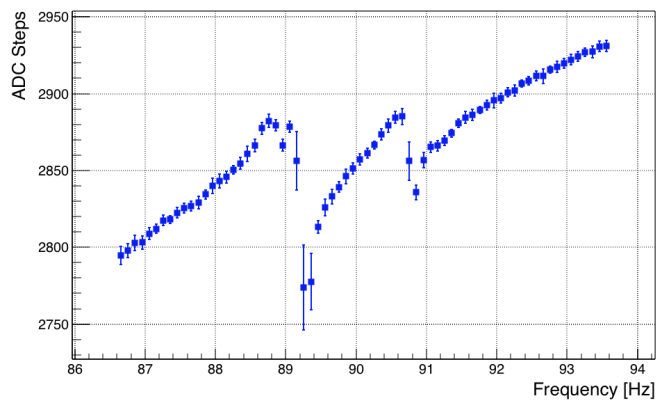

(a) $1.0 \mathrm{~m}$ long wires at $V_{0}=350 \mathrm{~V}, \frac{S}{N}=22.79$.

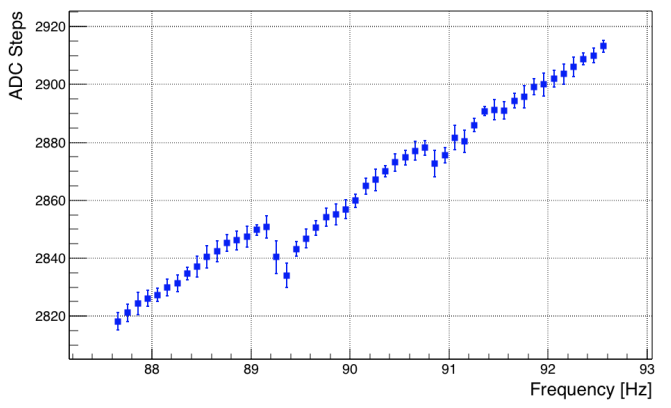

(b) $1.0 \mathrm{~m}$ long wires at $V_{0}=150 \mathrm{~V}, \frac{S}{N}=5.40$.

Figure 7.18: Comparison between beyond and below the SBND voltage limit for $1.0 \mathrm{~m}$ long segments. The larger peak corresponds to the wire being measured and the smaller peaks at the resonant frequencies of the adjacent wires.

When measuring the tension of shorter wires, the amplitude of the resonant peak becomes smaller because the excitation of the oscillation is more difficult. Figure 7.19 shows the result for $0.75 \mathrm{~m}$ long wires. For $\frac{S}{N}<3.5$ we found that it was difficult to infer the frequency from data and, since the ratio is smaller for smaller lengths, we set the lowest limit on the length to $0.75 \mathrm{~m}$. This limit is mainly driven by the limits on the voltages that can be applied on

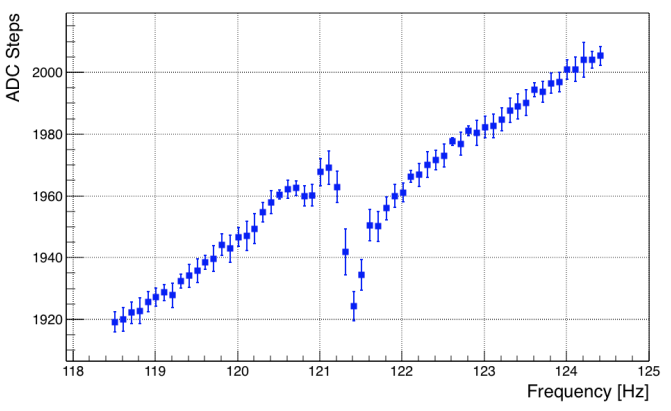

(a) $0.75 \mathrm{~m}$ long wires at $V_{0}=350 \mathrm{~V}, \frac{S}{N}=11.95$.

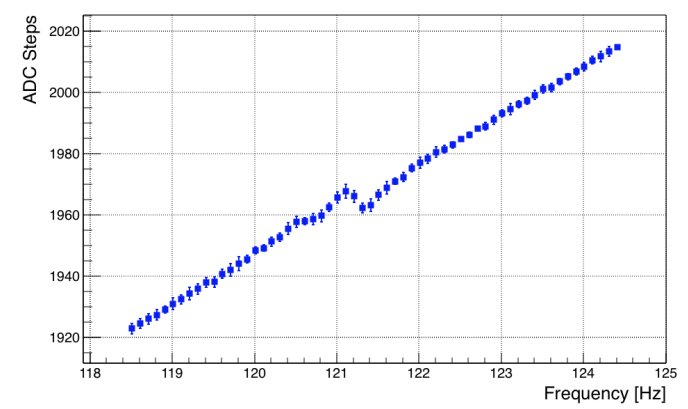

(b) $0.75 \mathrm{~m}$ long wires at $V_{0}=150 \mathrm{~V}, \frac{S}{N}=3.70$

Figure 7.19: Comparison between beyond and below the SBND voltage limit for $0.75 \mathrm{~m}$ long wires. At lower $V_{0}$ it becomes harder to distinguish the signal from the baseline.

the SBND boards. Therefore, it may not be possible to measure the shortest wires unless future electric boards allow using higher voltages. It is worth noting that the measurement of the shortest wires could also be challenging for the laser set-up: in the commissioning of 
the protoDUNE detector at CERN some issues connected to the geometry of the frame and the positioning of the laser head have been observed [51] that made it impossible to measure the shortest wires. In any case, we have demonstrated that the new approach works with the majority (about 90\%) of the SBND wire segments in the APAs. In future liquid argon experiments, like DUNE [52], it should be possible to design the electronic boards to allow for higher voltage biases. That would allow the tension of shorter wires to be measured by simply raising the voltages used.

\subsubsection{Resolution}

To understand the resolution of the technique, the long-frame was used together with the version $V 3$ of the circuit. The version $V 3$ implements the idea of $V 2$ to readout up to 32 channels using the CAEN digitizer. For practical reasons, only eleven $4 \mathrm{~m}$ long wires were soldered at $500 \mathrm{~g}$ and a comb was used to measure the tension of a $1 \mathrm{~m}$ long segment. The voltages used were $150 \mathrm{~V}$ for the constant bias and $40 \mathrm{~V}$ for the amplitude of the AC signal. Both voltages and wire length were chosen to be similar to the SBND conditions: $1 \mathrm{~m}$ is short enough to characterise the resolution for most of the segments (most of wire segments in SBND are longer than $1 \mathrm{~m}$ ) and results in a clear signal with voltages within the SBND requirements.

The measurement of each wire is plotted in the histogram of Figure 7.20. They are distributed with a mean value of $4.561 \mathrm{~N}(\sim 465 \mathrm{~g})$ and a standard deviation of $0.070 \mathrm{~N}$ $(7 \mathrm{~g})$. Our conclusion is that the electric method allows to determine the wire tension with a resolution of a several grams, better than $2 \%$.

\subsection{Calibration}

In order to study the bias of the electric method, we performed a cross-calibration with the laser method. The first step was to build a dedicated laser set-up and calibrate it with respect to the nominal tension of the wires. Then, the electric measurements were calibrated using the laser as reference and corrected for the laser calibration in order to obtain the calibration with respect to the nominal values. The reason of this procedure is that direct calibration of the electric technique is difficult: the wires need to be soldered on both ends to be connected to the circuit. This way, some tension can be lost during the soldering procedure, which is not needed for the laser method calibration. The calibration was performed using the version $V 2$ of the circuit. 


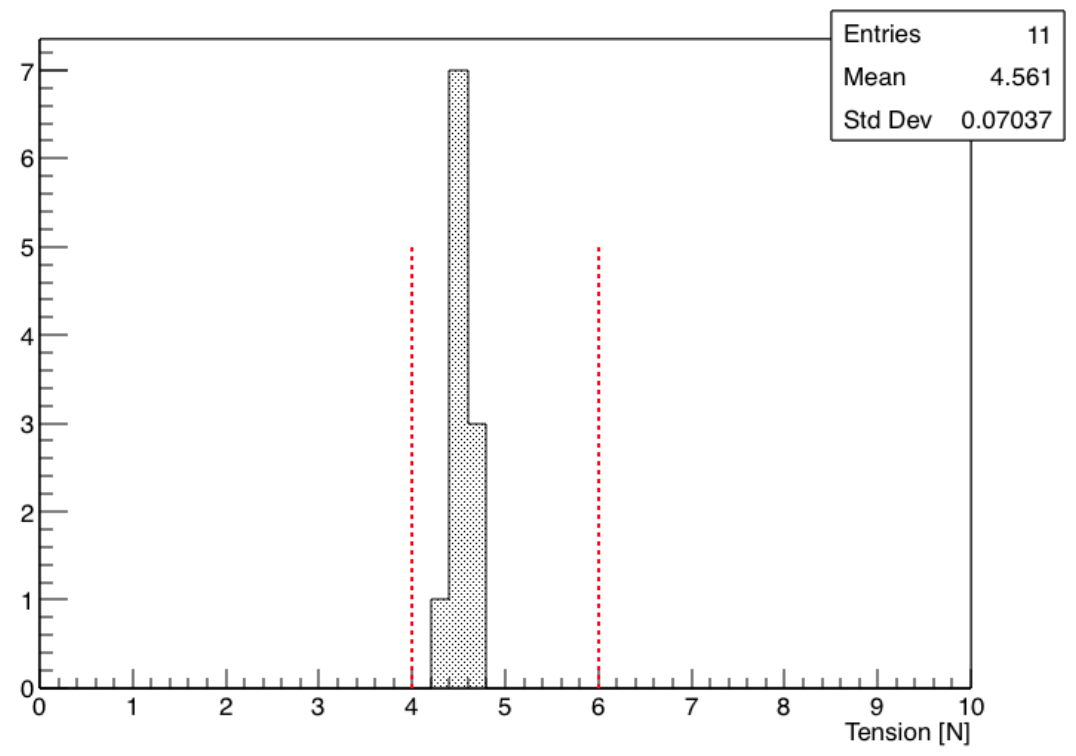

Figure 7.20: Resolution of the method for repeated measurements. The two red lines show the region allowed by the tolerance of $1 \mathrm{~N}[40]$.

\subsubsection{Laser set-up}

Figure 7.21 shows the set-up used to calibrate the laser.

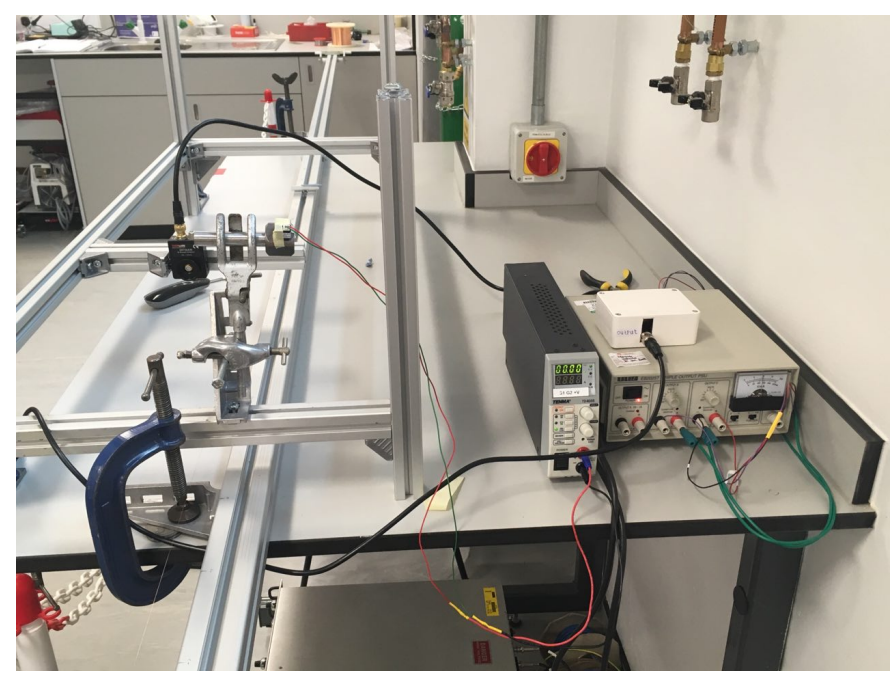

Figure 7.21: Laser set-up used to calibrate the wire tensioning measurement.

The laser was installed on a frame together with an off-axis photodiode. The light hit the wire with a certain angle and was reflected from the wire in a forward cone (Figure 7.22) to be caught by the photodiode.

The wires were mechanically perturbed to oscillate at their resonant frequency resulting in generating an electric signal with the same frequency from the photodiode. The signal was 


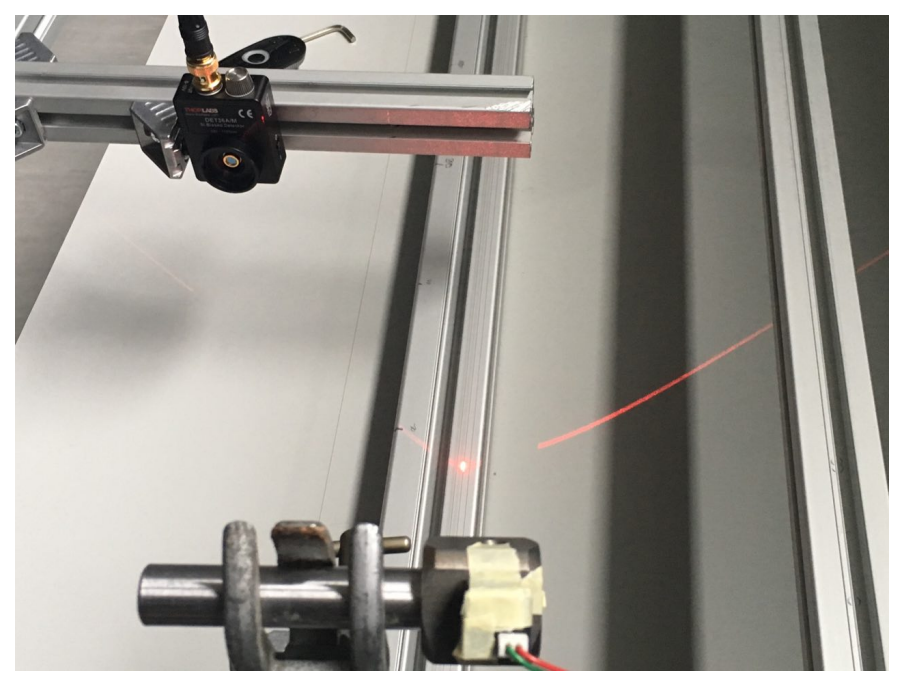

Figure 7.22: Light from laser (bottom) is reflected off the wire in the forward direction. In the plane perpendicular to that of the wire, the reflections pattern is a circumference.

then amplified by an amplifier (white box in right-hand side of Figure 7.21) and read out by a laptop through a jack plug. An acquisition script deconvolved the signal using a Fast Fourier Transform (FFT) to obtain peaks in the frequency distribution in corresponding to the resonant frequency and the harmonics. Figure 7.23 shows a typical event acquired by the LabVIEW DAQ script used to work out the value of the resonance.

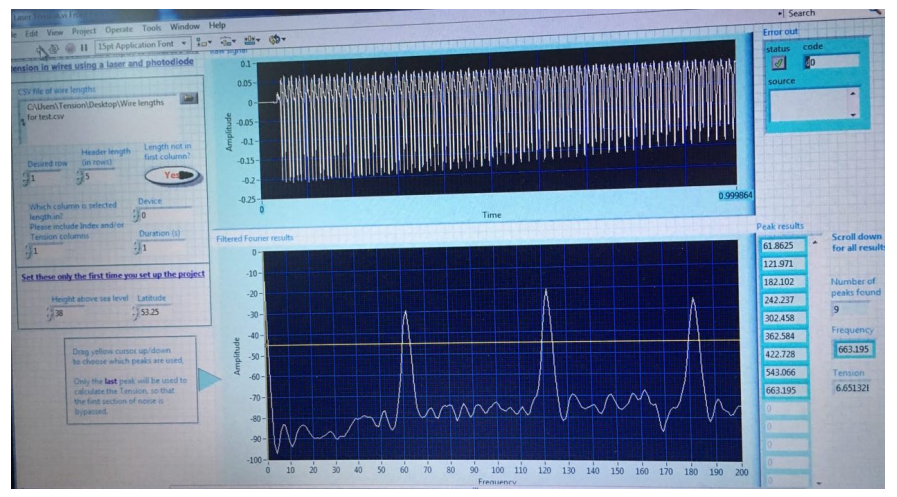

Figure 7.23: DAQ script for laser tensioning set-up. The upper part shows the pure signal amplitude as a function of time. The lower part show the signal deconvolved: there are three different peaks corresponding to the resonant frequency and the first two harmonics.

To calibrate the measurements, a single wire was prepared using the long-frame. One end was fixed by soldering, while the other end was tied to a weight of $500 \mathrm{~g}$ and left hanging from a pulley (just like in Figure 7.5). The calibration was done without soldering the second end of the wire to avoid altering the tension during soldering. The procedure was performed at different wire lengths by using combs (see Section 7.5.2). The error on the measurement of length was estimated as $\pm 2 \mathrm{~mm}$. Table 7.2 summarises the selected length 
and the relative nominal frequency, i.e. the expected resonant frequency for wires tensioned at $500 \mathrm{~g}$ according to Equation 6.1.

Table 7.2: Lengths and corresponding nominal frequency for the wires measured.

\begin{tabular}{cc}
\hline Wire length [m] & Nominal frequency $[\mathbf{H z}]$ \\
\hline \hline 0.5 & 181.12 \\
1.0 & 90.56 \\
1.5 & 60.37 \\
2.0 & 45.28 \\
2.5 & 36.22 \\
3.0 & 30.19 \\
\hline
\end{tabular}

Figure 7.24 shows the calibration of frequency measured with the laser with respect to the nominal frequency obtained from Equation 6.1, overlaid with a linear fit. The experimental points have vertical error bars given by the standard deviation of the set of measurements, but the laser technique is very precise and thus error bars are invisible on that scale. There are also error bars on the $x$-axis (not visible in the plot) given by the uncertainty on the length measurement used to obtain the nominal frequency from the nominal tension.

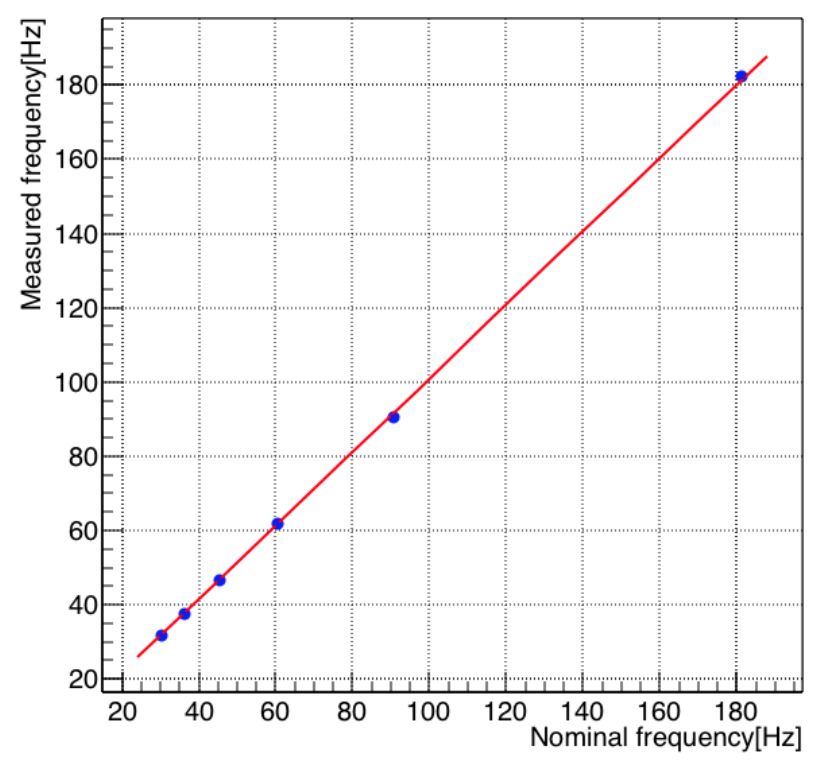

Figure 7.24: Calibration of laser measurements. The plot shows the experimental measurements performed with the laser set-up corresponding to the nominal frequency expected. The red line is obtained with a linear fit to the points.

The fit gives $m=0.990 \pm 0.003$ for the slope and $q=1.9 \pm 0.1 \mathrm{~Hz}$ for the offset. Figure 7.25 shows the difference in tension between the laser measurements and the expectations as a function of the length. Only the measurements of the segments with length $\lesssim 2 \mathrm{~m}$, i.e. 
the relevant lengths for SBND, are shown. The bias goes from a few grams to $30 \mathrm{~g}$ in the SBND region of interest. This is an acceptable result considering that the SBND tolerance is $100 \mathrm{~g}$ [40]. In order to parametrise the dependence of the bias on the wire length, a linear

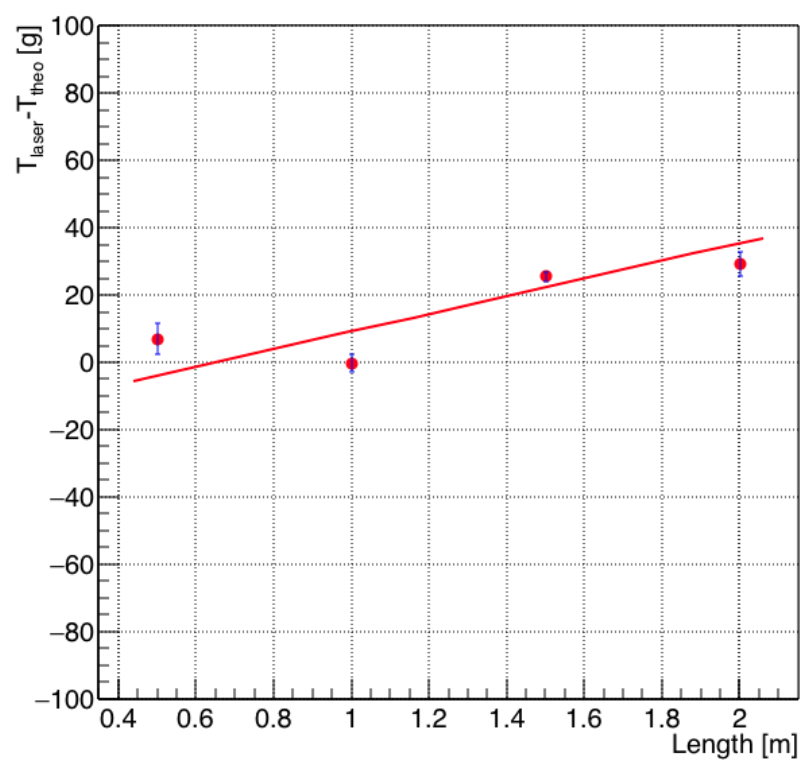

Figure 7.25: Bias of laser measurements as a function of wire length. Only the measurements of the segments with length $\lesssim 2 \mathrm{~m}$, i.e. the relevant lengths for SBND, are shown. The red line is obtained with a linear fit to the experimental points.

dependence was assumed and the points fitted (red line in Figure 7.25). The result of the fit is

$$
\begin{gathered}
m_{1}=(26 \pm 3) \mathrm{g} / \mathrm{m}, \\
q_{1}=(-17 \pm 5) \mathrm{g} .
\end{gathered}
$$

where $m_{1}$ is the slope and $q_{1}$ is the offset. This parametrisation was then used to relate the tension measured with the electric method to the nominal tension.

\subsubsection{Electric method calibration}

In order to take measurements with the electric circuit, it was necessary to solder the other ends of the wires. Together with the electric measurements, laser measurements were taken again and potential tension loss during the soldering of the wires could be corrected for by measuring the tension with the laser method and making use of the parametrisation of Equation 7.1.

Figure 7.26 illustrates the calibration plot between frequency obtained with the electric and the laser method. The segment lengths are the same as the ones used during the laser 
calibration with the exception of $0.5 \mathrm{~m}$ which was under the limit estimated for the electric method and therefore was replaced by $0.75 \mathrm{~m}$. The error on the electric measurement is given by half the step of the frequency scan: $0.05 \mathrm{~Hz}$. The blue line represents the bisector being drawn to show that the electric method systematically underestimates the frequency. The

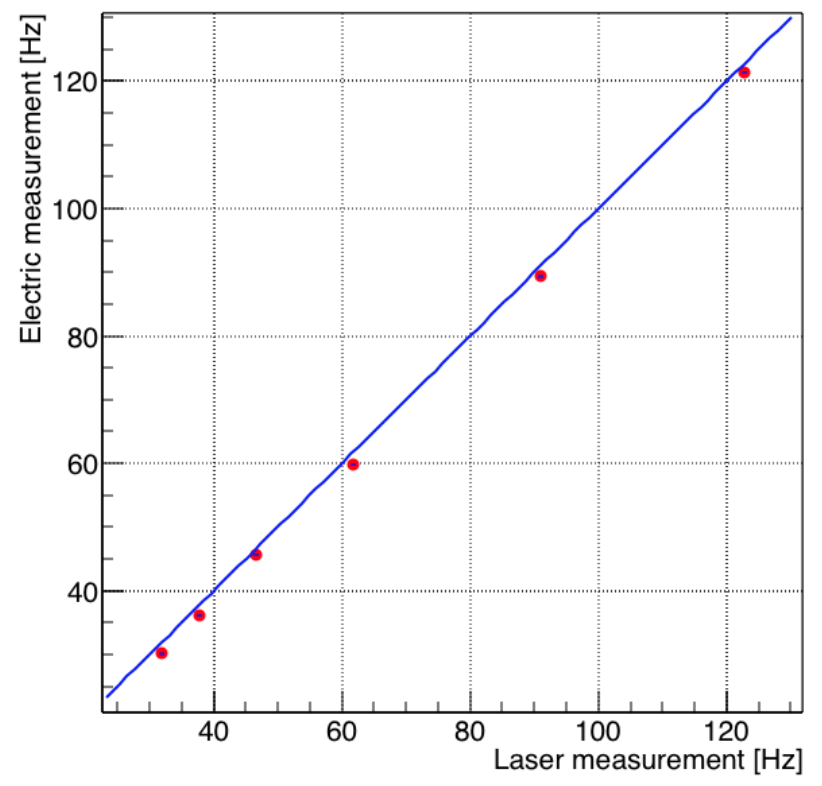

Figure 7.26: Calibration of electric measurements with respect to laser measurement. Point are plotted for the laser and electric measurement at different lengths. The blue line is the bisector, drawn to highlight the systematic underestimate of the electric method with respect to the laser.

values for the laser are corrected for the bias using the parameters $m_{1}$ and $q_{1}$ : the difference between the tension calculated with the laser $T_{\text {laser }}$ and the theoretical tension $T_{\text {theo }}$ is given as a function of the length $l$ by $T_{\text {laser }}-T_{\text {theo }}=m_{1} l+q_{1}$, while from data in Figure 7.26 is possible to calculate $\left.T_{\text {elec }}\right|_{l}-\left.\left.T_{\text {laser }}\right|_{l} \equiv \alpha\right|_{l}$ where $T_{\text {elec }}$ is the tension measured electrically and $\left.\alpha\right|_{l}$ is the difference worked out at every length $l$. Therefore, to correct for the laser bias, $T_{\text {elec }}-T_{\text {unbiased laser }}=\left.\alpha\right|_{l}+\left(m_{1} l+q_{1}\right)$. The plot of $T_{\text {elec }}-T_{\text {unbiased laser as a function of }}$ the frequency is shown in Figure 7.27 in the SBND segments length range as before. The plot demonstrates that the electric wire tension measurement can determine the tension of the wires within $\sim 20 \mathrm{~g}$ from their nominal value for wire length $<2 \mathrm{~m}$ being therefore well within the required specifications. 


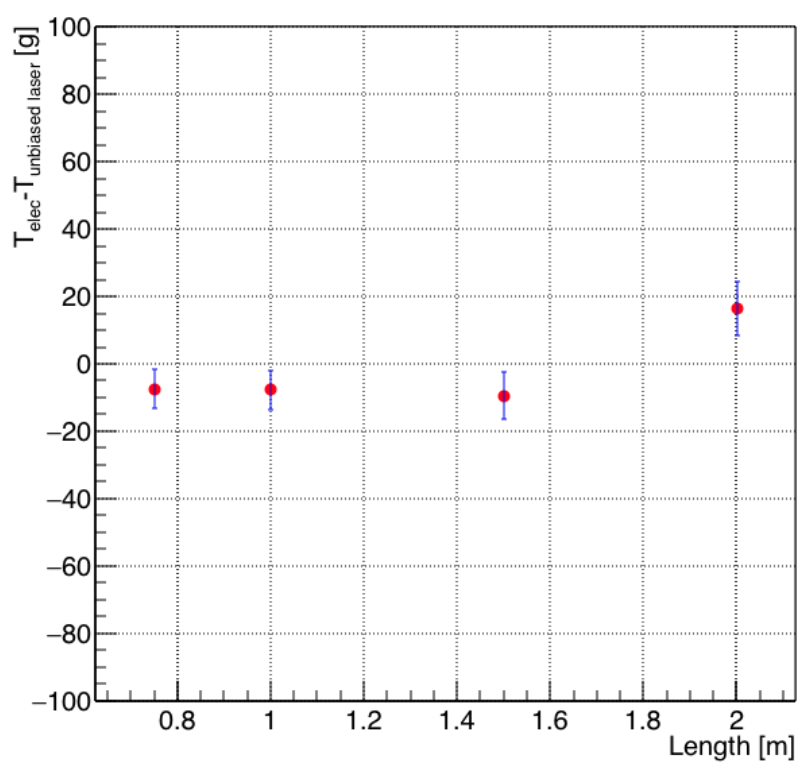

Figure 7.27: Tension bias between the electric and the corrected laser measurements.

\subsection{Test at cryogenic temperatures}

One of the main advantages of the electric technique is that it is in principle applicable in a closed cryostat and at cryogenic temperatures where length contractions could have changed the tensions in the system. We have tested this possibility in-situ using a setup consisting of a vacuum jacketed dewar and the short-frame. In order to obtain low temperature, a combination of gas nitrogen and liquid nitrogen $\left(\mathrm{LN}_{2}\right)$ was used. Liquid nitrogen has a boiling point of $-195.79^{\circ} \mathrm{C}$, very similar to that of the argon, $-189.34{ }^{\circ} \mathrm{C}$. The main limitation of the measurement is the presence of the liquid since it can damp the oscillation, but the measurement should be possible in cold gas, which is the first stage of LArTPC filling. Therefore, in the measurements described in this section we made sure to never immerse the wire in the liquid, otherwise higher voltages would have been needed. Thus, the values we were able to use were $350 \mathrm{~V}$ for the constant bias and $40 \mathrm{~V}$ for the AC signal amplitude.

\subsubsection{Experimental set-up}

The experimental set-up consisted of the already mentioned short-frame with copper-beryllium wires, a vacuum jacketed dewar and four PT1000 temperature sensors. A photo of the set-up (inside of the dewar) is shown in Figure 7.28. The $V 2$ version of the circuit was used. The frame was firmly fixed on the wall of the dewar and the four temperature sensors were tied 


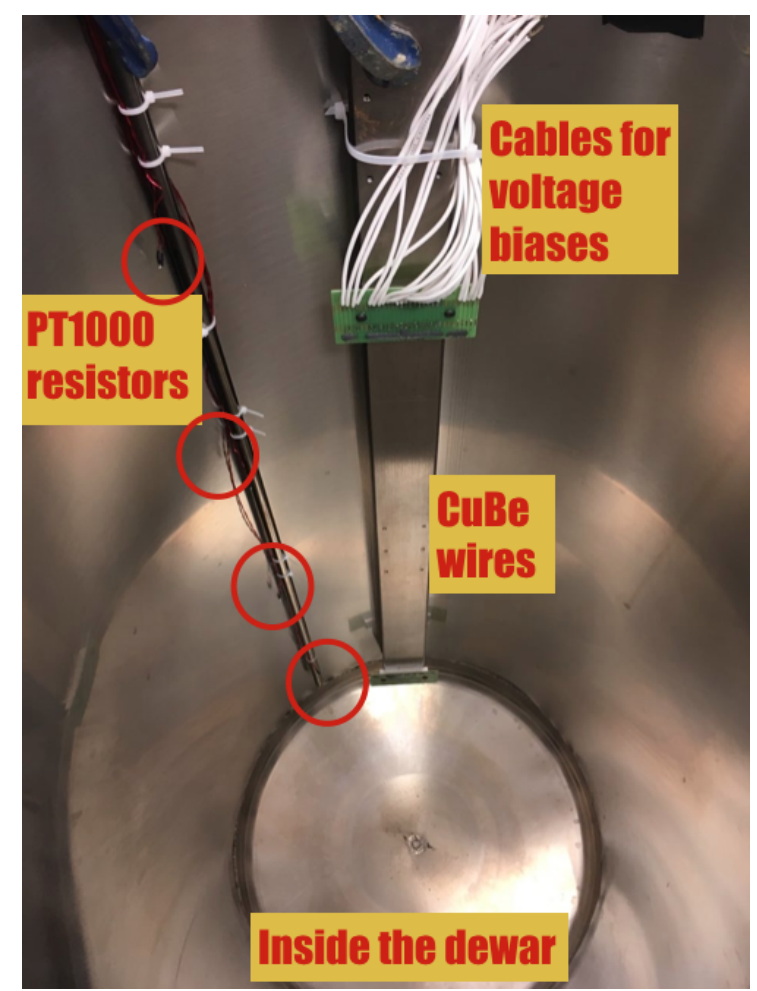

Figure 7.28: Cold test set-up.

to a metal rod that was fixed along the frame. This allowed the monitoring of the temperature at different heights in the dewar in order to take the tension measurement only when the temperature along the wires is relatively uniform. For this reason the lower sensor was positioned slightly below the lower end of the wires and the upper sensor was positioned just above the upper end.

\subsubsection{Measurement procedure}

Maintaining a stable gas temperature was challenging because of the instability of the set-up due to a high heat load. Figure 7.29 presents the behaviour of the temperature as a function of time (Channel 1 being the lowest sensor and Channel 4 being the highest) for two relatively stable periods of time, when we took the tension measurements.

Given the limitations of the set-up, we decided to take tension measurements when the temperature difference between the upper and the lower sensors were $\leq 50{ }^{\circ} \mathrm{C}$ (both continuous and dotted black vertical lines in Figure 7.29). At a later time, we were able to perform the analysis only with measurements where the difference was $\leq 30{ }^{\circ} \mathrm{C}$ (continuous black lines in Figure 7.29). The two relatively stable configurations were obtained by first filling the bottom of the dewar up to the lower end of the wires with liquid nitrogen. This easily provided a sudden drop of the temperature, but left a large difference between the sensors 


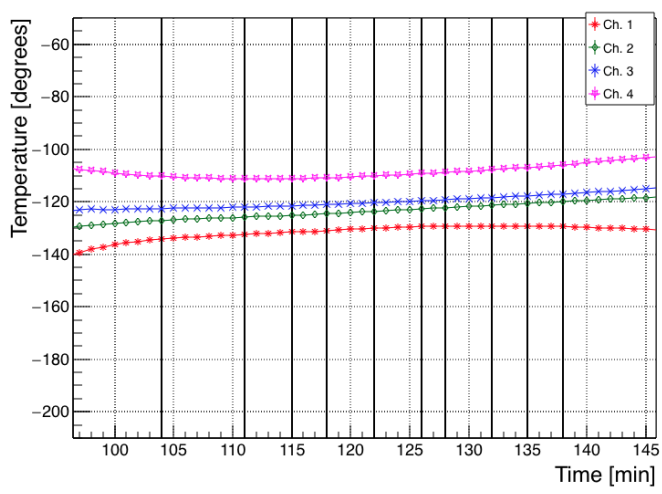

(a) First stable region.

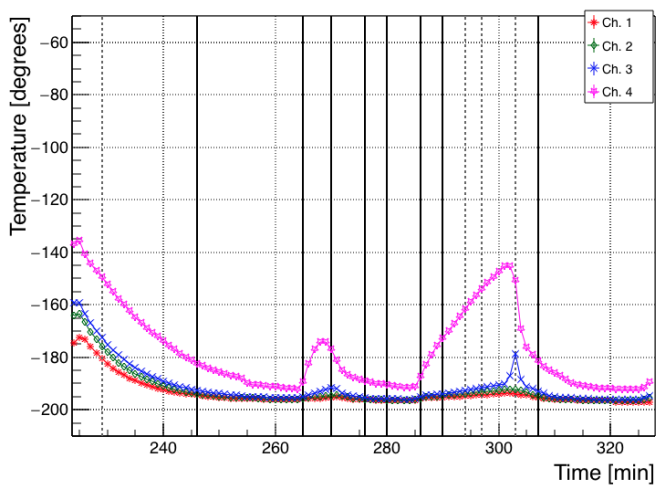

(b) Second stable region.

Figure 7.29: Temperature vs. time for each of the four sensors. The bumps in the plot on the right are caused by the dewar lid being opened to check the level of the liquid. The vertical black lines are drawn corresponding to the time when a measurement was taken. See text for further description.

at the extremes. Uniformity was achieved by injecting gas nitrogen from the bottom of the dewar (Figure 7.29(left)) and injecting liquid nitrogen at the brim (Figure 7.29(right)). The temperature was continuously monitored and the flux of gas or liquid adjusted to stabilise the temperature gradient.

\subsubsection{Expectations and analysis}

The material of the frame (stainless-steel) and wires were chosen by the SBND collaboration to have very similar thermal coefficients. Therefore, a large change in tension due to different stretching of the pieces was not expected. Under this assumption a change in frequency would only come from a change in length (see Equation 6.1), due to the thermal contraction of the entire set-up. Given that the thermal expansion coefficient of copper-beryllium is $\alpha \sim 17 \cdot 10^{-6}{ }^{\circ} \mathrm{C}^{-1}$, the relative change of the length is $\left|\frac{\Delta L}{L}\right|=|1+\alpha \Delta T|=0.3 \%$ where $\Delta T \simeq-210{ }^{\circ} \mathrm{C}$ is the difference between room temperature $T_{0} \simeq 20^{\circ} \mathrm{C}$ and the temperature of liquid nitrogen $T_{\mathrm{LN}_{2}} \simeq-190^{\circ} \mathrm{C}$. Hence, a change in frequency of at most $\left|\frac{\Delta f}{f}\right| \simeq 0.3 \%$ was expected.

Figure 7.30 shows the measurement of the resonant frequency as a function of the temperature. The frequency values were obtained with the fit procedure and the error bars are given by the temperature difference between the two sensors at the extremes of the wire length. The red dots represent data, while the blue line is the expected and extrapolated behaviour assuming only a change in length.

There is a clear discrepancy between data and expected behaviour that becomes even more 
Frequency vs. temperature

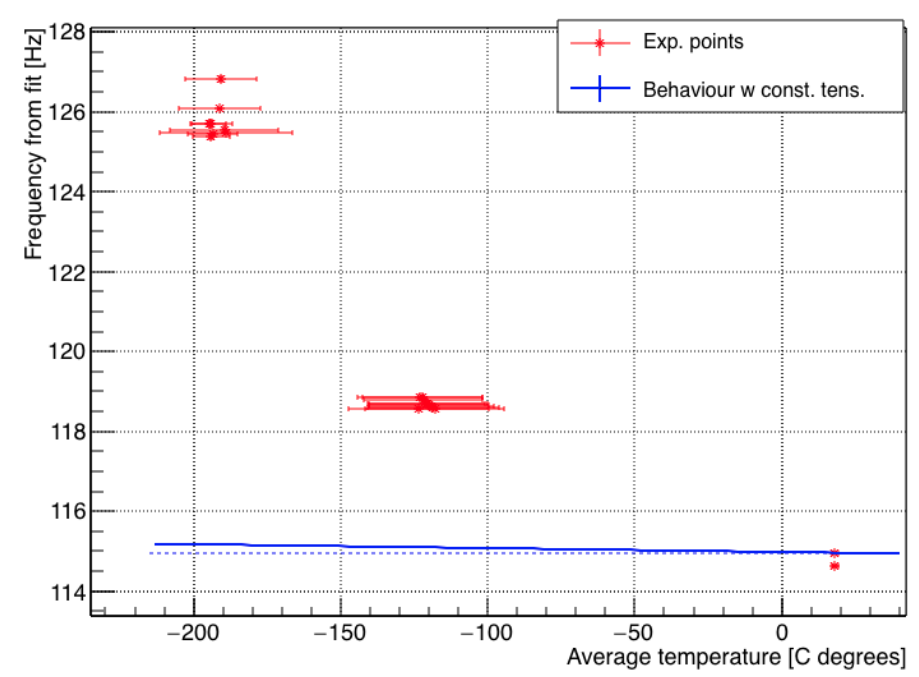

Figure 7.30: Frequency vs. temperature measured during the cold test. Data (red points) is larger than the expected resonant frequencies of the wires (continuous blue line). The dashed blue line was drawn to highlight the small slope of the continuous one.

evident after translating the frequency into tension (see Figure 7.31). In this case, the

Tension vs. temperature

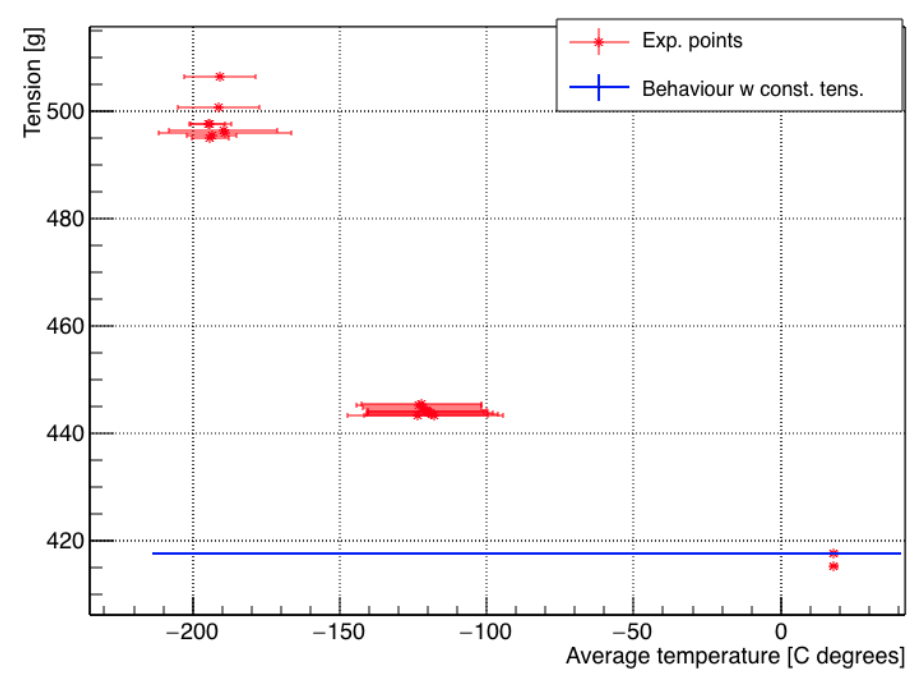

Figure 7.31: Tension vs. temperature measured during the cold test. Data (red points) is larger than the expected constant tension of the wires (blue line). Details in the text.

blue line becomes flat and it is possible to estimate the difference between the expected tension and the measured values: for measurements around $-125^{\circ} \mathrm{C}$ the difference is $26 \mathrm{~g}$ and for those around $-190{ }^{\circ} \mathrm{C}$ it is $82 \mathrm{~g}$. This leads to a conclusion that there are additional temperature effects affecting tension in our set-up.

A bar that contracts less could explain the observed behaviour: the frame was not entirely 
contained in the dewar and therefore its temperature is expected to be higher than that of wires in at least part of its length. If this were the case, the tension of the wires could have increased because they were at lower temperature than the bar and therefore they contracted more. To understand the plausibility of this hypothesis, a simple model can be assumed: suppose that the upper end of the bar was at room temperature $T_{0}$, the lower end was at $T_{\mathrm{LN}_{2}}$ and that a linear temperature gradient exists between the two extremes. The temperature along the frame would be $\Delta T(l)=\beta l$ where $\beta=\frac{\Delta T_{\max }}{L}$ with $\Delta T_{\max }=-210^{\circ} \mathrm{C}$ and $L$ being the length of the frame. Using the reference system defined in Figure 7.32, it is possible to integrate the linear contraction formula to obtain the change in length of the bar segment covered by the wires.

The length of the segment $L_{\text {seg }}^{\prime}$ after contraction would then be

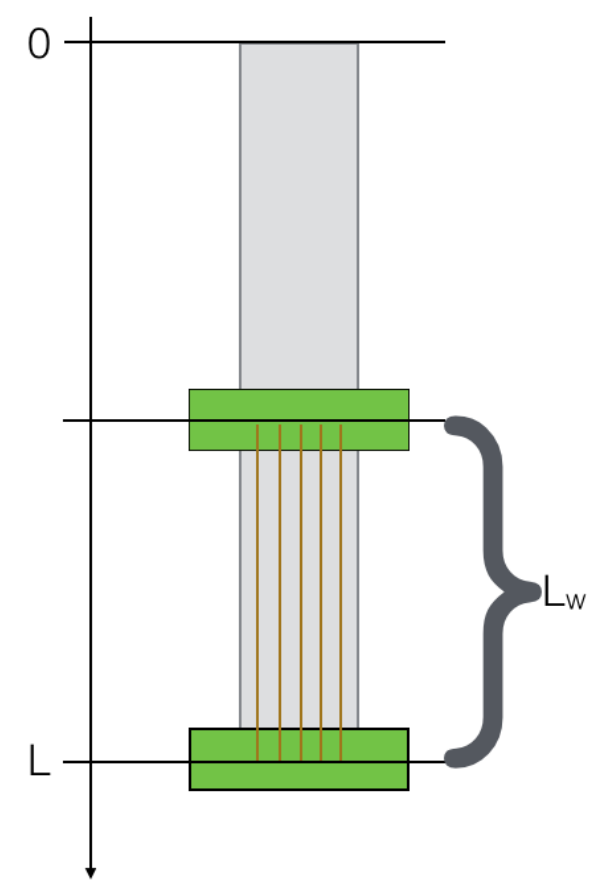

Figure 7.32: Schematic of the short-frame and reference system.

$$
L_{\mathrm{seg}}^{\prime}=\int_{L-\left|L_{w}\right|}^{L}(1+\alpha \Delta T(l)) d l=\int_{L-\left|L_{w}\right|}^{L}(1+\alpha \beta l) d l
$$

where $L_{w}$ is the wire length. Hence:

$$
\begin{aligned}
L_{\mathrm{seg}}^{\prime} & =[l]_{L-\left|L_{w}\right|}^{L}+\frac{\alpha \beta}{2}\left[l^{2}\right]_{L-\left|L_{w}\right|}^{L} \\
& =\left|L_{w}\right|\left[1-\frac{\alpha}{2}\left|\Delta T_{\max }\right|\left(2-\frac{\left|L_{w}\right|}{L}\right)\right] .
\end{aligned}
$$

Since in our set-up $\frac{\left|L_{w}\right|}{L} \approx \frac{1}{2}$

$$
L_{\mathrm{seg}}^{\prime}=\left|L_{w}\right|\left[1-\frac{3}{4} \alpha\left|\Delta T_{\max }\right|\right] .
$$


This is an extreme case: in the real situation there is a likely smaller difference in the temperature between the two ends of the bar. The difference between the length after contraction of the bar in the wire region $L_{\mathrm{seg}}^{\prime}$ and the wire length after contraction $L_{w}^{\prime}$, $\bar{\Delta}=L_{\mathrm{seg}}^{\prime}-L_{w}^{\prime}$, is

$$
\begin{aligned}
\bar{\Delta} & =\left|L_{w}\right|\left(1-\frac{3}{4} \alpha\left|\Delta T_{\max }\right|\right)-\left|L_{w}\right|\left(1-\alpha\left|\Delta T_{\max }\right|\right) \\
& =\frac{1}{4}\left|L_{w}\right| \alpha\left|\Delta T_{\max }\right| \\
& =0.0006048 \mathrm{~m} .
\end{aligned}
$$

This gives an effective relative change in length of

$$
\frac{\bar{\Delta}}{\left|L_{w}\right|}=0.9 \% \text {. }
$$

The Young's modulus $Y$ for copper-beryllium is 130 GPA, meaning $Y=130 \mathrm{~N} / \mathrm{mm}^{2}$. Taking the radius of the wires, $r=75 \cdot 10^{-3} \mathrm{~mm}$, the force of the contracting wires that acts on the bar would increase by the value $F$ :

$$
F=\pi r^{2} Y \frac{\bar{\Delta}}{\left|L_{w}\right|} \simeq 200 \mathrm{~g} .
$$

The value obtained is a factor of 2.5 greater then the measured $82 \mathrm{~g}$. This is likely a consequence of the approximations: perhaps the temperature gradient is not linear along the bar and the temperature is more uniform than assumed. Another possible reason why this model overestimates the change in tension could be the assumption that the temperature on the wires is uniform.

However, it does lead to the conclusion that the tension change we observed can be explained by difference in temperatures of the steel frame and wires. Our measurement shows that such changes could occur during a cool down of the TPC and that they could be monitored in "real time" using the electric tension measurement.

\subsection{SBND dedicated development}

\subsubsection{Comparing glue/solder methods}

The short-frame set-up was also used to test different methods of fixing wires on the boards to determine the best method for the SBND detector using wires prepared at $500 \mathrm{~g}$. According to the proposal of the SBN program [24], wires are bonded soldering the wire on gold pads and applying a layer of epoxy. Our goal was to understand which combination of glue/soldering was the best in terms of loss in tension with time, at least in the short period. In order to 
do so, the system presented in Figure 7.33 was prepared with the following configurations: wires only soldered to gold pads, soldered close to the edge of the board plus epoxy applied, soldered far from the edge plus epoxy and same configurations but with the wires cut after the solder pad, so that they are only kept in tension by the glue.

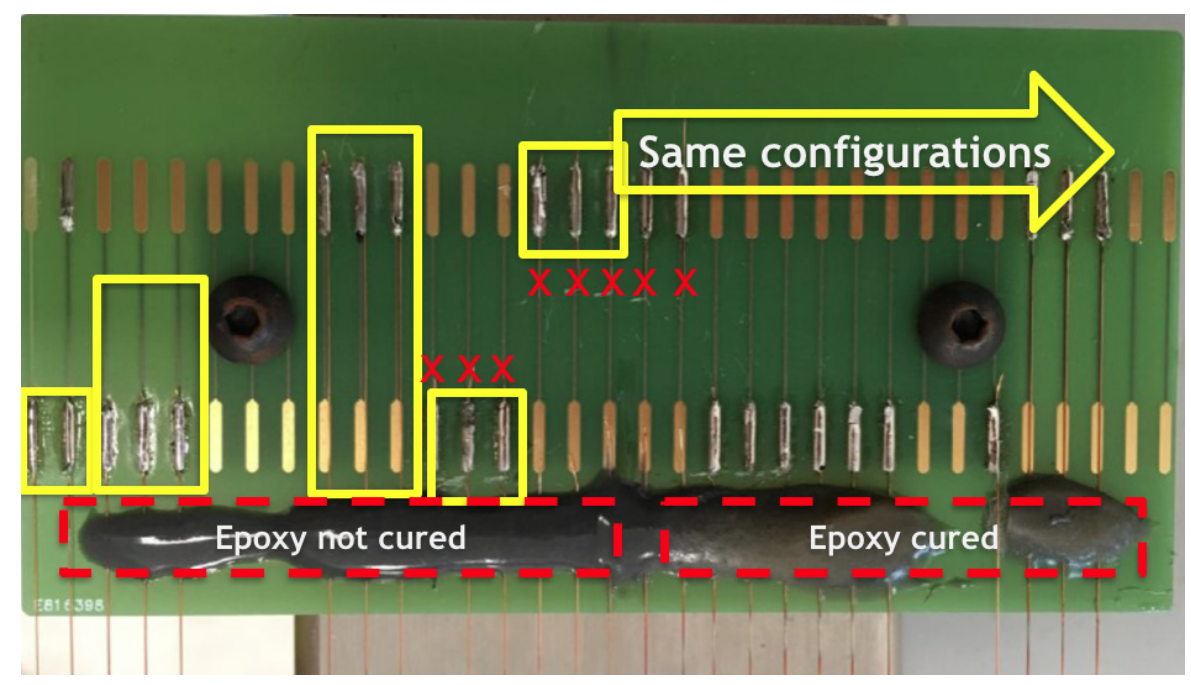

Figure 7.33: Set-up for bonding technique tests. From left to right yellow boxes: only soldered wires, wires soldered close to the glue, wires soldered far from the glue, wires soldered close to the glue and then cut after the glue cured, wires soldered far from the glue and then cut after the glue cured. The same structure repeats on the right but in this case the glue was cured with a heat gun.

For each configuration, there were two versions: in one case the epoxy was cured right after the application with hot air, in the other case the epoxy was left to cure by itself.

The circuit version $V 1$ was used in this case, since at the time $V 2$ was not developed yet. As a consequence, the value of the constant voltage needed was relatively high, $1.25 \mathrm{kV}$, as expected given the short length of the wires. The amplitude of the AC signal was $10 \mathrm{~V}$. An example of the signals is shown in Figure 7.34.

The tension was measured three times for each pair of wires right after the set-up was prepared, and the same measurements were taken again after two weeks. The differences in tension are plotted in the histogram of Figure 7.35. They are plotted in the same histogram since no sensitive loss in tension was observed and for every configuration the difference in tension was well within the tolerance. We concluded that all bonding methods were sufficiently strong on the time scale of these measurements. 


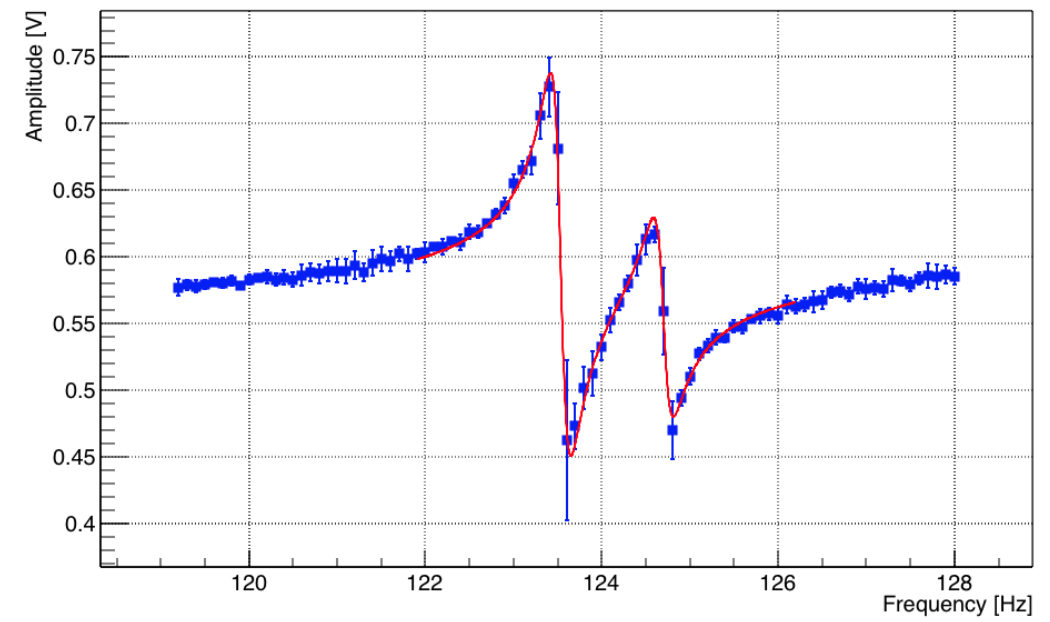

Figure 7.34: Signal for two $72 \mathrm{~cm}$ long wires with slightly different resonant frequencies (two peaks). The tension in grams corresponding to the frequency estimated by the fit is $(482.3 \pm 2.7) \mathrm{g}$ for the first peak and $(491.5 \pm 2.7) \mathrm{g}$ for the second one.

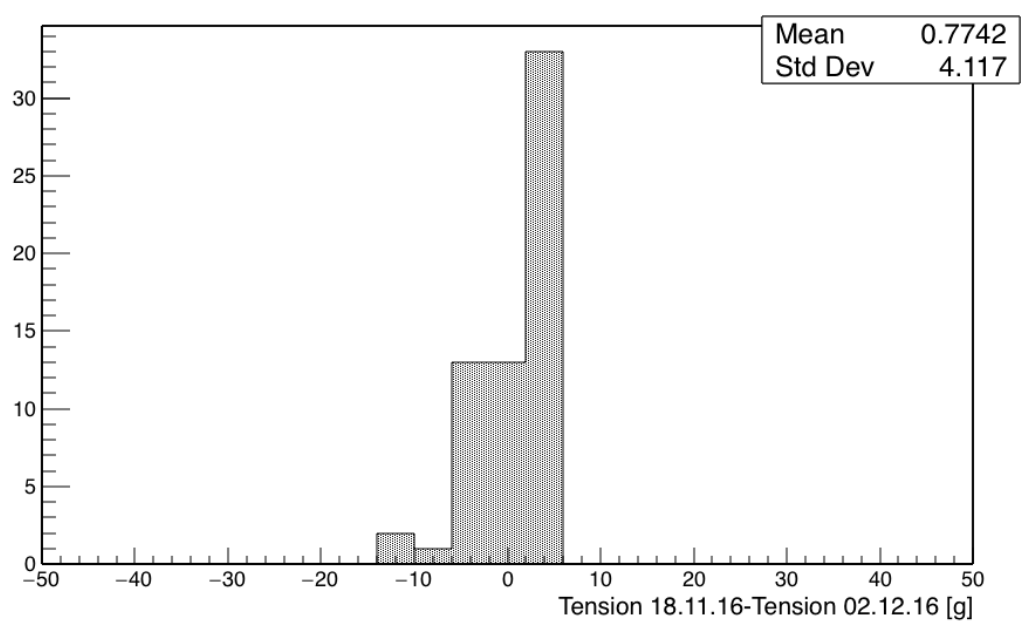

Figure 7.35: Loss in tension after two weeks. 


\subsubsection{Measurements with combs}

An important test of the technique was to see its performance in case of wires divided by combs. This is one of the main advantages over the laser method, which requires measurements of each segment separately, while in the case of the electric method the measurement would be on a wire-per-wire basis instead of segment-by-segment. SBND and the DUNE experiment will be using combs as a method of minimising the risk from breaking wires and therefore it was important to understand whether the electric technique could work in this kind of set-up.

The measurements with combs were taken using the long-frame. One $4 \mathrm{~m}$ long wire was prepared at $300 \mathrm{~g}$ and a second at $500 \mathrm{~g}$ tension, and the length divided in half by a comb. The result is given in Figure 7.36: two peaks at around $35 \mathrm{~Hz}$ corresponding to the two segments at $300 \mathrm{~g}$ and two peaks around $45 \mathrm{~Hz}$ corresponding to the two segments at $500 \mathrm{~g}$. The uncertainty on the position of the combs, which translates in two segments of slightly different length, and the resolution of the method cause the frequencies to be not identical (four peaks are visible instead of two).

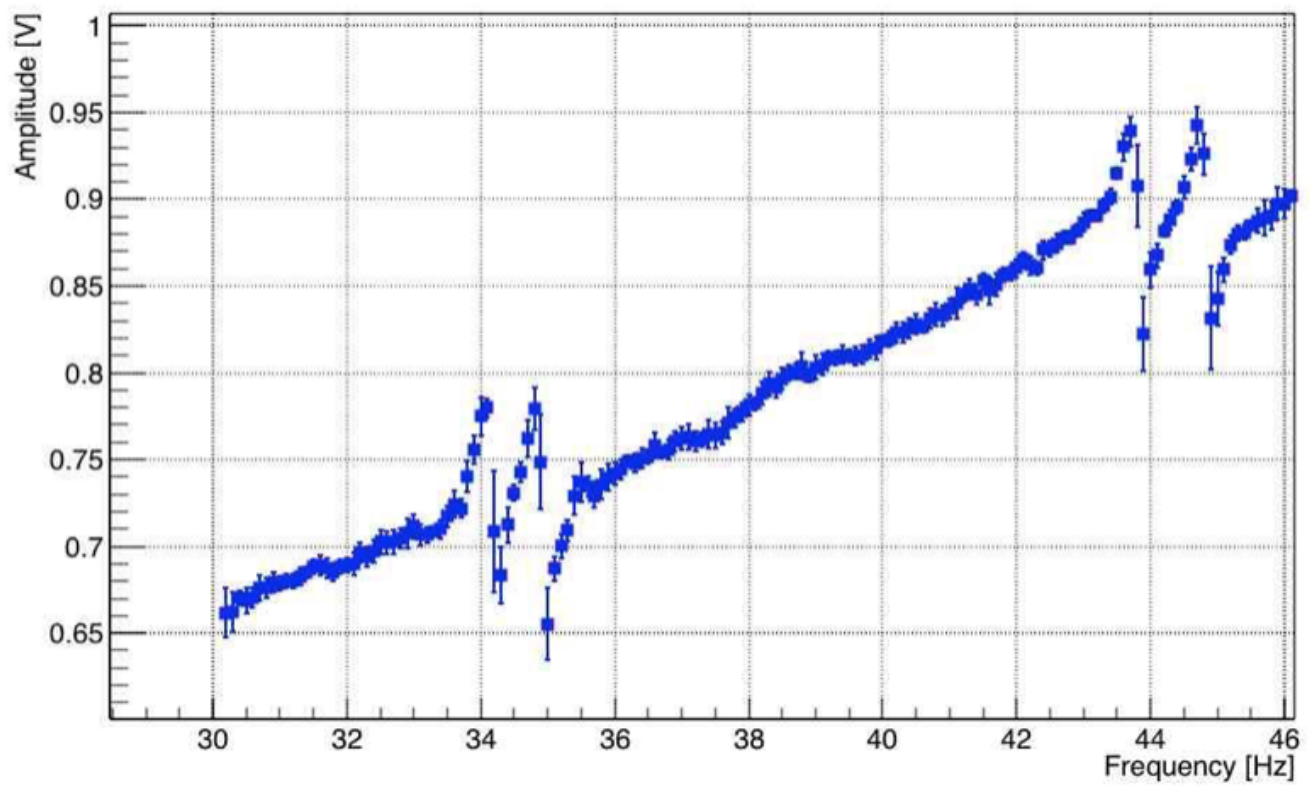

Figure 7.36: Example of measurements with combs.

\subsubsection{Test of 32-channel readout with test frame}

The final test of the set-up was to use it to measure the tension of 32 wires simultaneously. A possible concern was whether induced signals between wires could affect the measurement. 
This test also served as an important step to connect the circuit to the real boards. The version $V 3$ of the circuit was used.

Figure 7.37a shows the circuit board on top of the CAEN digitizer and the connection for the board on the frame prepared at Daresbury Laboratory (Figure 7.37b). The board

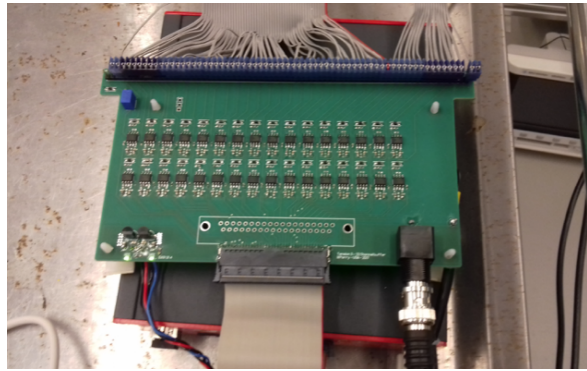

(a)

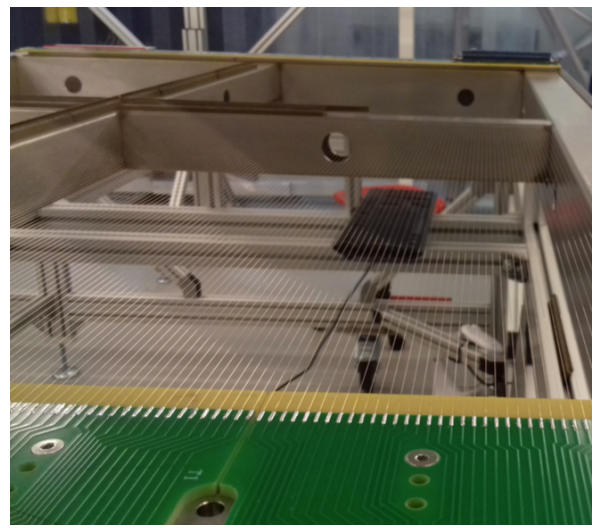

(b)

Figure 7.37: Photos of set-up for 32 channel readout. The CAEN digitizer together with the electronic circuit board (left) and the SBND mock-up frame (right) are shown.

has 66 pins in total: 32 are for the readout of the signal of the wire to measure, 33 are to bias the adjacent wires $(32+1$ because the far-left and far-right wires have to be biased) and another pin to get the signal from the "antenna". The connections on the board are shown in Figure 7.38. Figure 7.39 shows the signals for the first eight channels and Figure 7.40

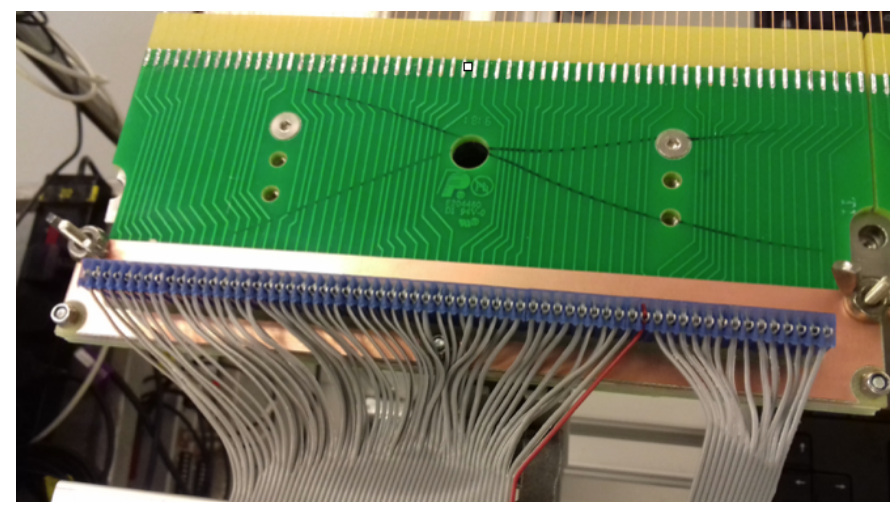

Figure 7.38: Connections to the SBND board for electric wire tension measurement.

show the signals for channel 9 to 16 . We demonstrated that the technique could work, but there are some improvements to make since some channels had smaller or no signal. A more effective grounding of the circuit could improve the results as well as a better electric isolation of the cables. At the time of the writing, this is still under development. 


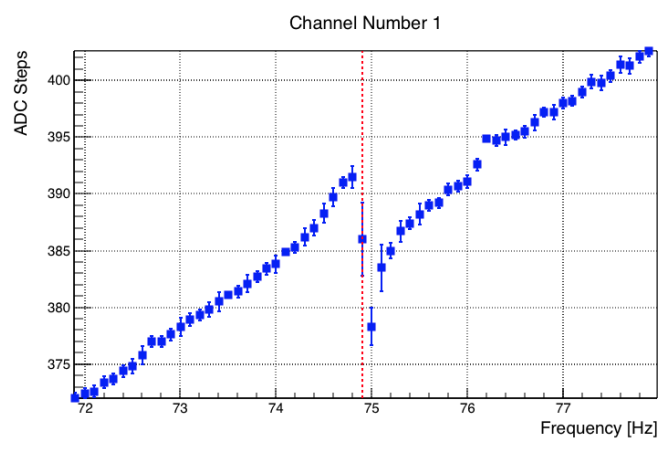

(a) Channel 1 .

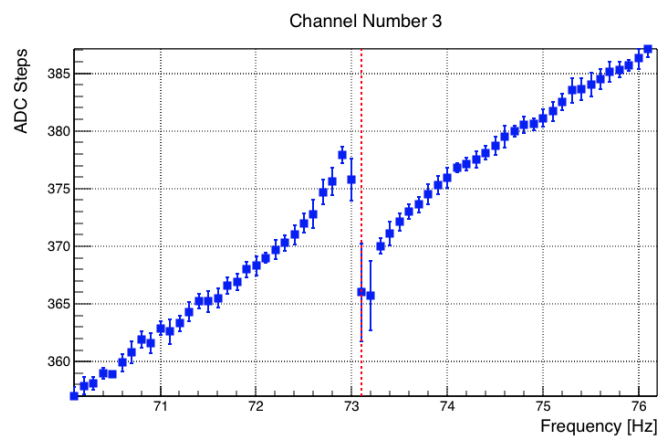

(c) Channel 3.

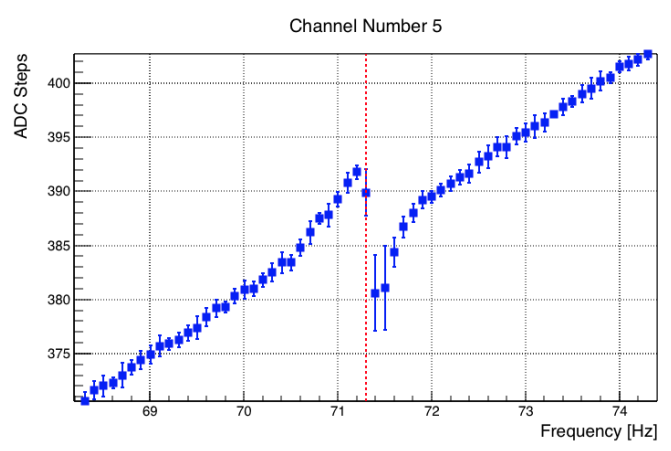

(e) Channel 5 .

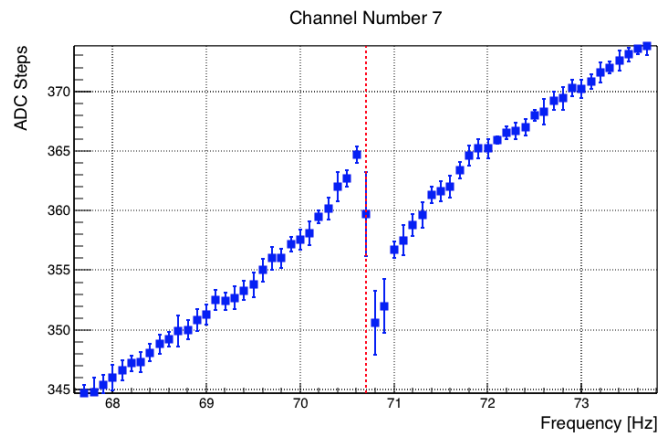

(g) Channel 7.

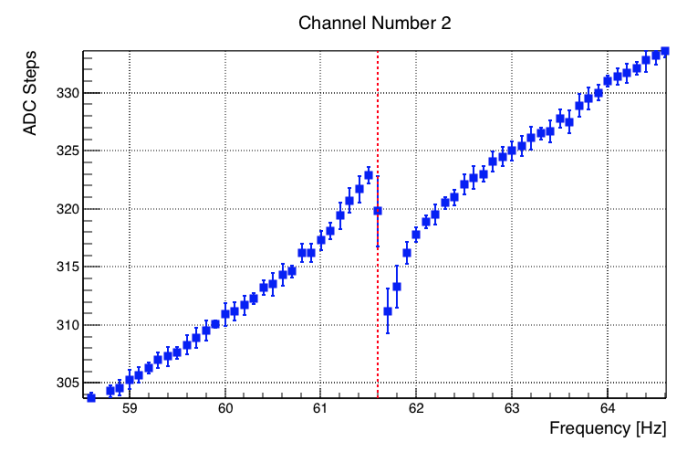

(b) Channel 2.

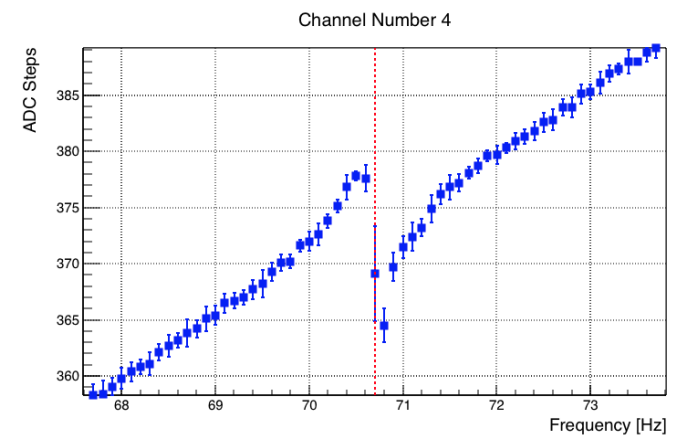

(d) Channel 4 .

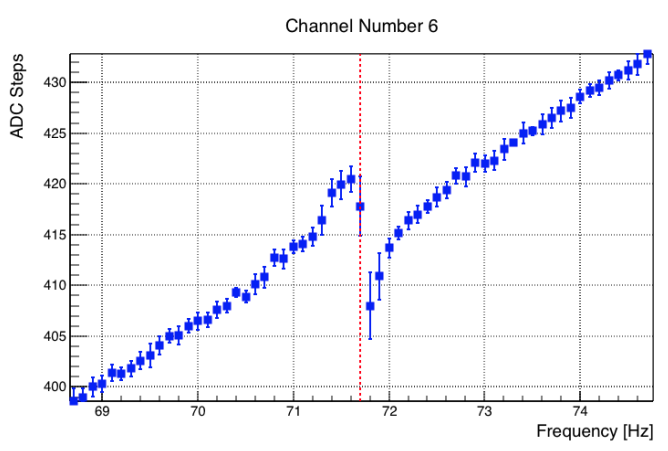

(f) Channel 6 .

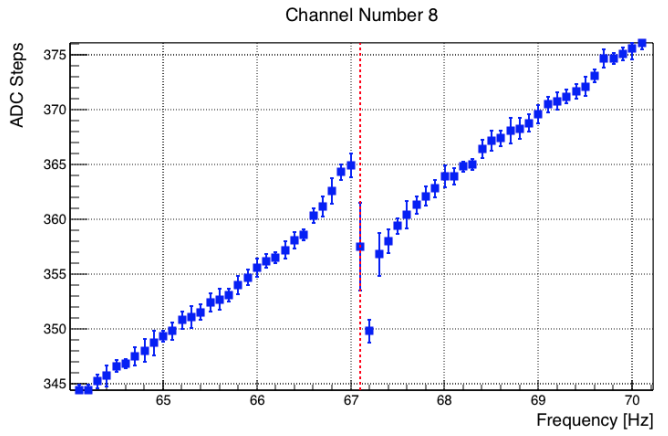

(h) Channel 8.

Figure 7.39: Data of the first 8 channels of the 32 channel acquisition performed at Daresbury Laboratory with the SBND mock-up frame. The red dashed vertical line was drawn in correspondence of the estimated resonant frequency. The voltage values for the measurement were $V_{0}=150 \mathrm{~V}$ and $v_{0}=40 \mathrm{~V}$. 


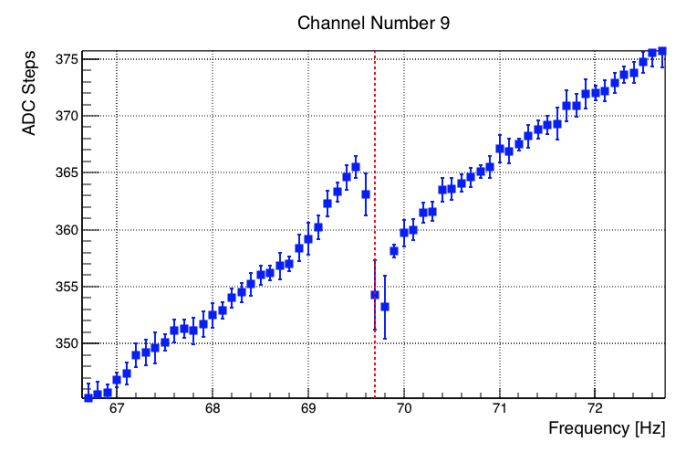

(a) Channel 9.

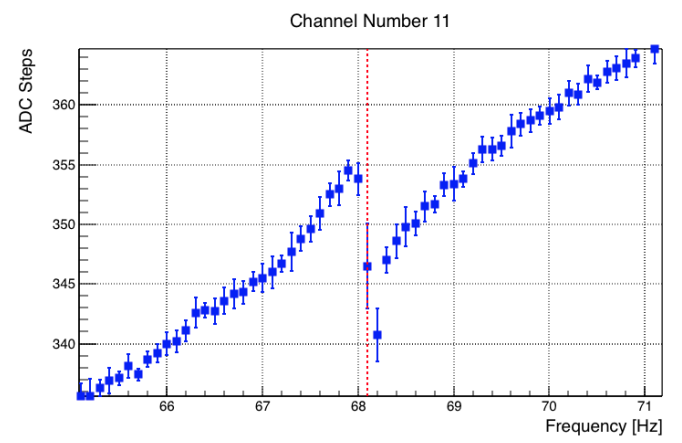

(c) Channel 11.

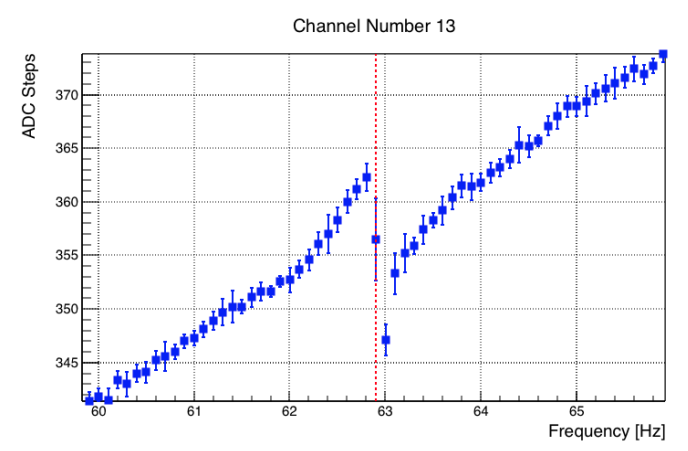

(e) Channel 13.

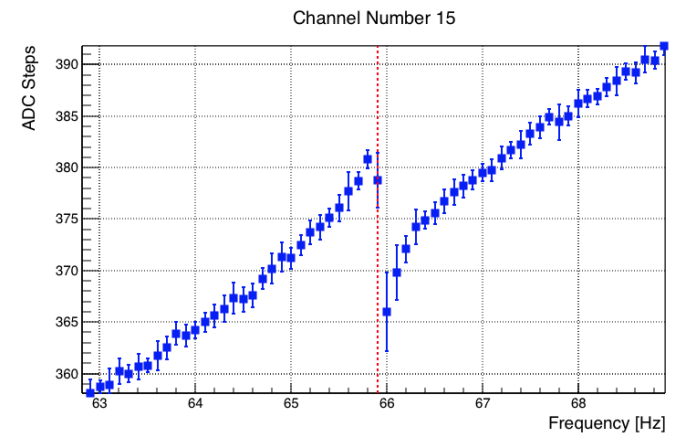

(g) Channel 15.

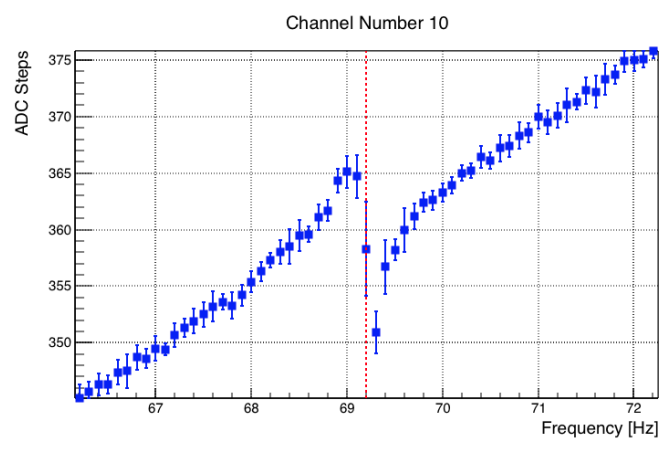

(b) Channel 10.

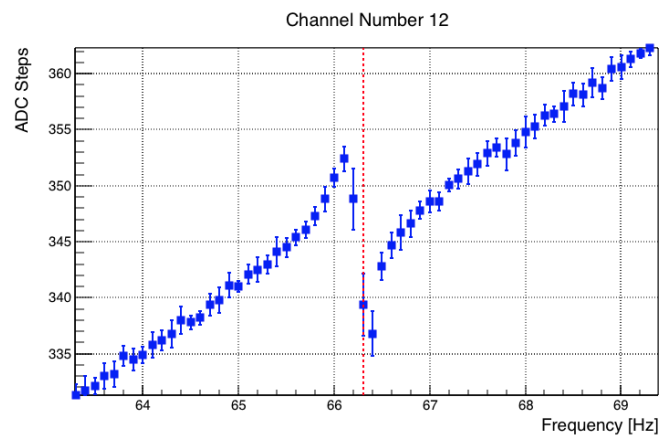

(d) Channel 12.

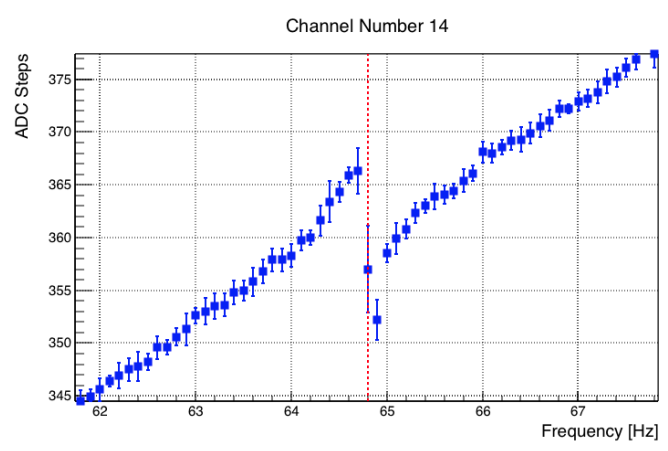

(f) Channel 14 .

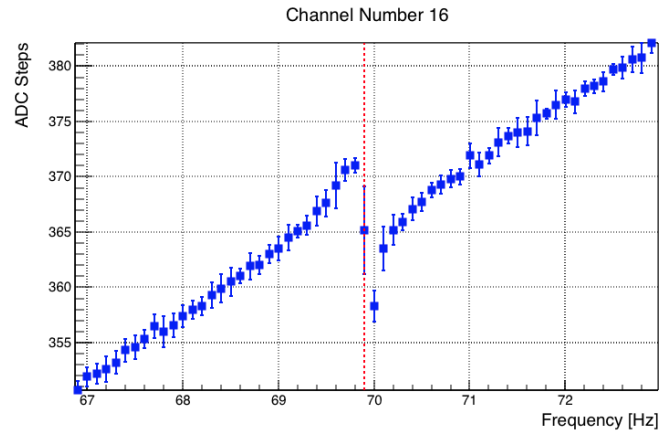

(h) Channel 16.

Figure 7.40: Data of channel 8 to 16 of the 32 channel acquisition performed at Daresbury Laboratory with the SBND test frame. The red dashed vertical line was drawn in correspondence of the estimated resonant frequency. The voltage values for the measurement were $V_{0}=150 \mathrm{~V}$ and $v_{0}=40 \mathrm{~V}$. 


\subsection{Conclusions}

We have developed a system which allows to measure the tension of multiple wires with a good signal-to-noise ratio using voltages of few hundreds volts. The main limitation is the current design of the SBND electric board which puts a strict constraint on the voltages that can be used. Future liquid-argon experiments could take this into account when designing the electric boards. Currently, there are studies ongoing to reduce the time necessary to perform the scan. These studies are focusing on a faster analog-to-digital converter and using optical link connections instead of USB interface.

The electric method has the advantage of not requiring to physically disturb the wires and allows to perform measurements in situations in which it would be impossible for the laser: measure of wires at the bottom of the APA or installed already in the TPC and cooled down with gas argon. It can also be much faster than the laser method, thanks to the fact that it can be scaled to potentially any number of wires measuring the tension of all the segments at the same time. We have shown that wires longer than $75 \mathrm{~cm}$ (90\% of the SBND wires) can be measured with a resolution of a few grams.

The electric technique passed an SBND readiness review and is the first wire tension measurement tested at cryogenic temperature. It will be used for SBND during construction and commissioning and has been proposed for the protoDUNE detector at CERN. 


\section{Chapter 8}

\section{Developing Wavelength-Shifter}

\section{Covered Reflector Foils for}

\section{LArTPCs}

\subsection{Introduction}

Scintillation light can be exploited to enhance the performance of LArTPC neutrino detectors, carrying information complementary to that of charge. Dedicated simulation studies have shown that increased precision in energy reconstruction, timing and position reconstruction is achievable in a high light yield LArTPC [53].

Monte Carlo simulations performed for the SBND experiment have shown [54] that wavelength-shifter (WLS) covered reflector foils inside the LArTPC active volume provide a more efficient and uniform light collection that allows to trigger on events in the whole active volume. Later simulations [53] showed that uniformity of light collection (see Figure 8.1) and energy resolution are preserved if reflector foils only cover the cathode. Since visible light travels faster than the VUV light in liquid argon, an early wavelength-shifting of light can improve the time resolution of the detector, because the faster-travelling visible light would mitigate the dispersion of the arrival time resulting from the intrinsic width of the light emission distribution [53]. It has been also shown that the difference of arrival time between visible and VUV light can be exploited by the light collection system to determine the position of charge deposition with a resolution of $\sim 12 \mathrm{~cm}$ [53], without the information of charge. It is possible to exploit this new feature to reject non-beam backgrounds. SBND will be equipped with PMTs sensitive only to visible light, i.e. without WLS coating applied, 
making this feature viable in the detector.

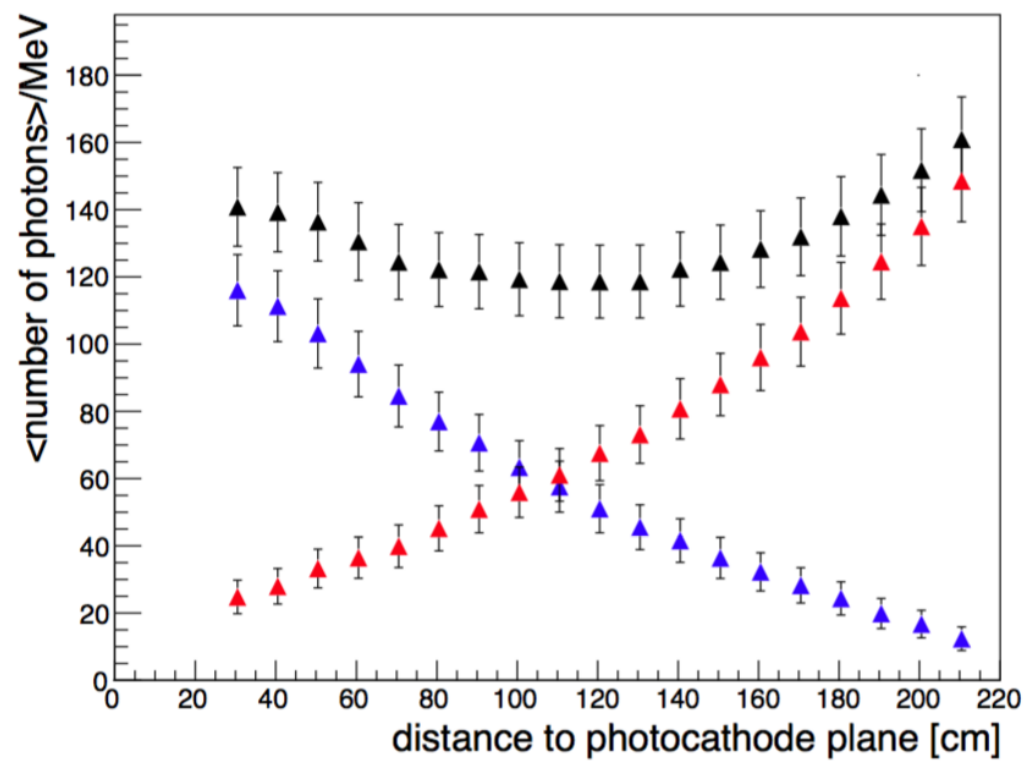

Figure 8.1: Average number of detected photons per $\mathrm{MeV}$ along the centre of the TPC as a function of the distance from the photocathode plane in the configuration with WLS-covered reflector foils covering the cathode. Blue points represent the VUV light directly detected by the PMTs, red points represent wavelength-shifted light reflected off of TPB-coated foils ("visible" light). The black points represent the total light collected (VUV+visible) [53].

These results have led to a proposal to install WLS-coated foils in the SBND detector. This chapter presents the work performed to develop the procedure and the feasibility of installation of a set of foils in SBND through tests performed in Manchester and using the LArIAT detector at Fermilab. These consisted of choosing the foil substrate that best suits the use in liquid argon and the installation requirements and defining the procedure to evaporate the foils with TPB, the WLS compound. A large scale evaporator was set up for this purpose. The defined evaporation procedure was then used to evaporate foils that were installed in a SBND-like mesh cathode (like the one described in Section 5.1.1) designed and manufactured in Manchester. The cathode was installed in the LArIAT detector in order to test the installation details and the behaviour of a LArTPC with a light collection system enhanced by wavelength-shifter coated reflector foils on the cathode.

\subsection{Testing substrate foils}

The key element of the proposed system for SBND are di-electric foils (VIKUITY [55]) or DF2000MA [56]. VIKUITY foils, normally used for cell-phone displays, are made of a thin organic multilayer material that has a total reflectance between $98 \%$ and $100 \%$ for visible 
wavelength above $400 \mathrm{~nm}$ on both sides. DF2000MA foils are usually exploited in commercial and industrial lighting applications and have optical properties very similar to those of VIKUITY foils. The main difference between the two kind of foils is that DF2000MA have one adhesive side. Also, DF2000MA are sold at a significantly lower price than VIKUITY. Both products are equipped with protective layers for the reflective side. In order to finalise the procedure and components of the SBND foil installation, a series of dedicated tests was performed. This allowed to choose the foil type and mounting method.

\subsubsection{Material Test Stand tests}

The material test stand (MTS) at Fermilab [57] was developed with the goal of testing the suitability of materials for their use in LArTPC detectors. More specifically, the tests show whether a material affects the purity of liquid argon and, as a consequence, the lifetime of the drifting electrons (see Section 4.3). The MTS operates in the following stages, here briefly illustrated (for further details see [57]). The supply argon is purified from water through a molecular sieve and is then sent through activated copper to eliminate oxygen contamination. The so-purified argon enters in a cryostat that is designed such that a specimen of material can be placed inside without emptying it from liquid argon. The cryostat is equipped with a purity monitor, based on the design explained in [58], which determines the electron lifetime.

The glued DF2000MA and VIKUITY foils were tested in the Material Test Stand (MTS) at Fermilab to understand if the glue could affect the electron drift time. Glue could influence the electronegative impurities in liquid argon resulting in a less effective collection of drifting electrons. The result of the test is shown in Figure 8.2 [59]. Comparing with the same measurements taken without the foils (Figure 8.3), it can be seen that the nominal lifetime flattened out at $4 \mathrm{~ms}$, similar to what happens in the foil test. We therefore concluded that neither of the foils had any impact on the liquid purity.

\subsubsection{Testing foil behaviour at LAr temperatures}

A series of tests were performed in order to understand the behaviour in cold of different potential foil mounting methods on the SBND cathode. This required understanding the interplay between the following components.

- Reflector foils.

- Mesh: a stainless-steel mesh which forms the actual cathode (i.e. the metallic electrode biased at HV) [60]. The mesh could be used to help the foil to stay fixed. In fact, two 


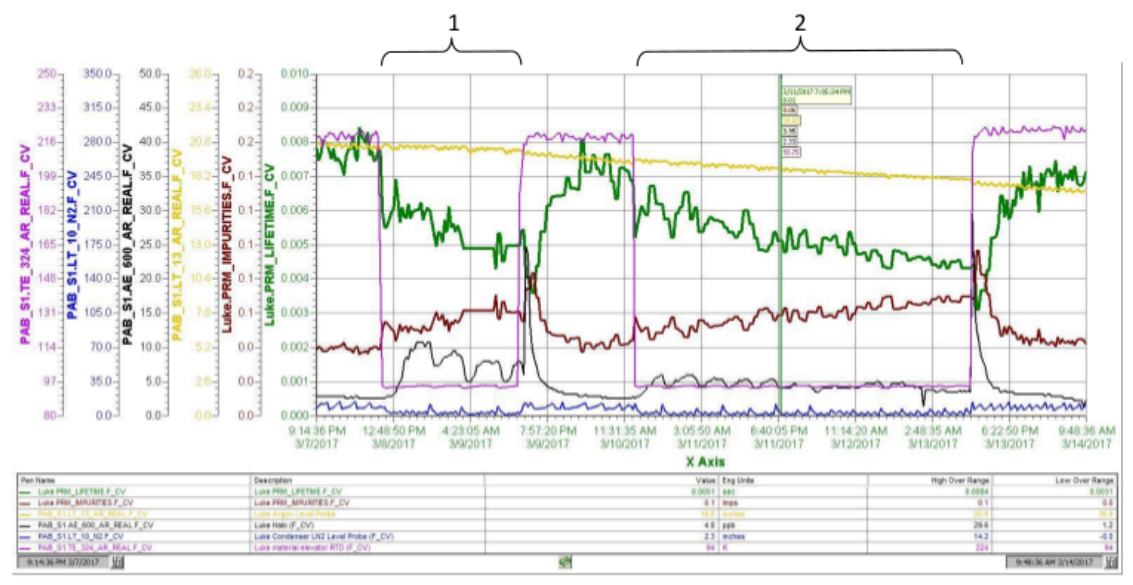

Figure 8.2: Result of the electron drift time with glued reflector foils. The green pen represents the lifetime $[\mathrm{s}]$ of drifting electrons, the brown pen represents the level of impurities [Imps] [59]. Note the plateau at $4 \mathrm{~ms}$. Numbers 1 is foil with adhesive and 2 is foil laminated on FR4 (see text later).

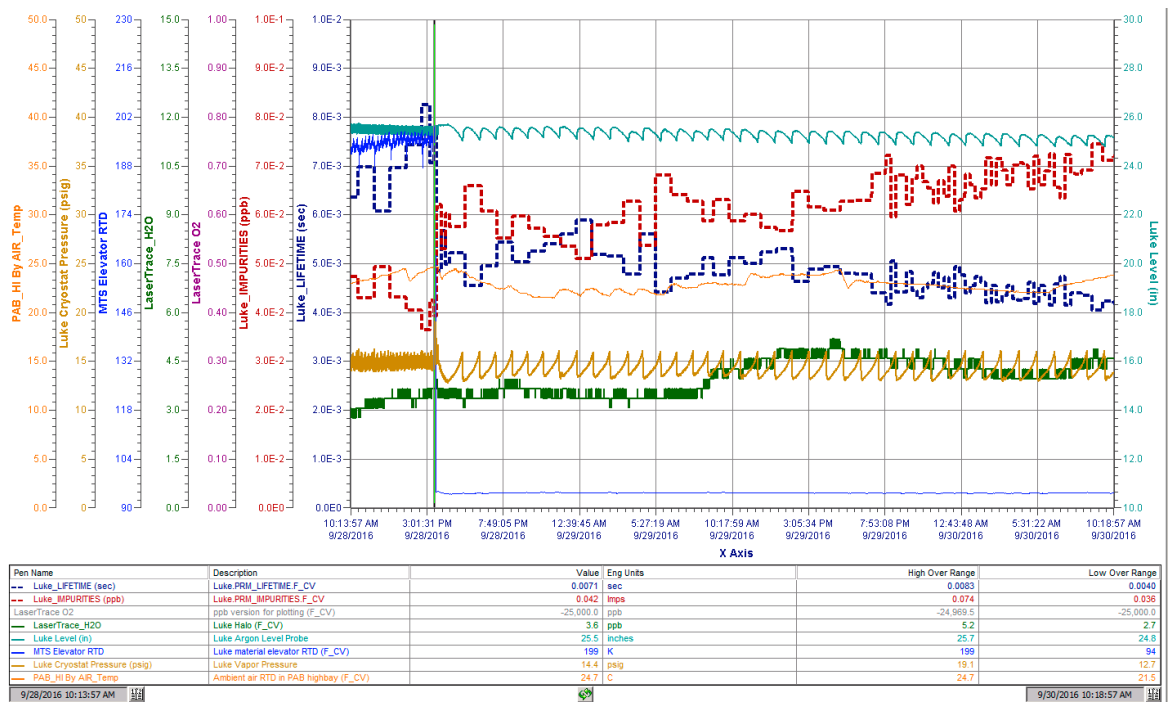

Figure 8.3: Result of the electron drift time without glued reflector foils. The green pen represents the lifetime $[\mathrm{s}]$ of drifting electrons, the brown pen represents the level of impurities [Imps]. Note the plateau at $4 \mathrm{~ms}$. 
back-to-back foils need to be squeezed between two layers of mesh (the SBND field cage is divided in two identical TPCs).

- Frame: a stainless-steel frame of size $25 \mathrm{~cm} \times 30 \mathrm{~cm}$ was built (see Figure 8.4 ). The frame had six screws that allowed to fix the mesh in the position.

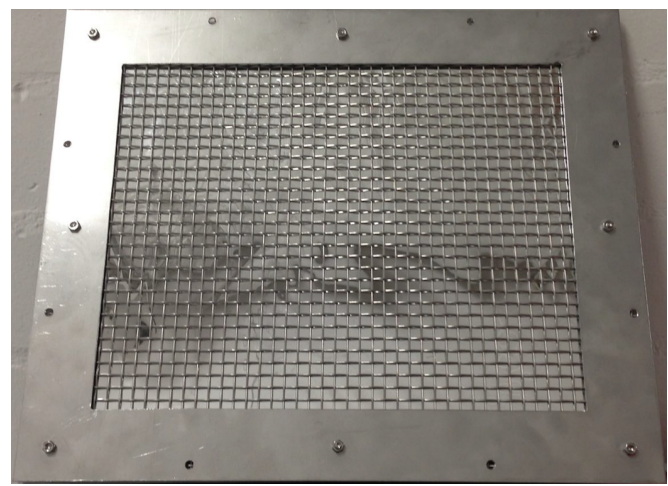

Figure 8.4: Set-up with small frame, mesh and foils built to test the behaviour of foils in liquid nitrogen.

To reproduce the very cold temperature of LAr, we used Liquid Nitrogen $\left(\mathrm{LN}_{2}\right)$ in an open dewar. Nitrogen and Argon have very similar properties at low temperatures. Table 8.1 summarises the main properties of the two elements.

Table 8.1: Properties of LAr and LN2.

\begin{tabular}{ccc}
\hline TPC Property & LAr & LN2 \\
\hline \hline Atomic number & 18 & 7 \\
\hline Melting point & $-189{ }^{\circ} \mathrm{C}$ & $-210{ }^{\circ} \mathrm{C}$ \\
\hline Boiling point & $-186{ }^{\circ} \mathrm{C}$ & $-196{ }^{\circ} \mathrm{C}$ \\
\hline Density & $1.40 \mathrm{~g} / \mathrm{cm}^{3}$ & $0.808 \mathrm{~g} / \mathrm{cm}^{3}$ \\
\hline
\end{tabular}

The following dunking tests were performed:

- Two DF2000MA foils glued back-to-back. The goal of this test was to understand the feasibility of the glueing procedure. Air bubbles can form between the two layers affecting uniform reflectivity and lead to the bump be scratched by the mesh. They could in principle change their shape (and get bigger) due to thermal contraction or extension of the material, air included.

- Single DF2000MA foil. This was done to understand the interplay of the three different materials that constitute the foil (the reflective material, the glue layer and the cover sheet for the glue) in case the background protective layer were to be used as a stiffening layer, which would minimise the bubbles from glueing back-to-back. 
- Two DF2000MA foils placed back-to-back (but not glued) sandwiched between the frame and the mesh. This set-up is the most similar to the real situations and was designed to understand if the mesh could scratch the foil surface and if the pressure from the screws on the frame was enough to keep the foils in the position.

- Same as the previous point, but with two halves of DF2000MA. This set-up was realised to test the extreme condition in which the foils are placed far from the frame and therefore the pressure coming from the screws that fix the frame is minimal.

- Laminated foils. The adhesive side of the DF2000MA foils allows the lamination of the foil material on different types of substrates. R\&D tests have been conducted by laminating DF2000MA material onto both sides of a FR4 substrate, resin-impregnated fibreglass material similar with properties similar to those of G10.

The rest of the section is dedicated to the description of these tests.

\section{Two DF2000MA foils glued back-to-back and single DF2000MA}

The foils were dunked in $\mathrm{LN}_{2}$ and a total of 3 cycling tests have been performed. They were left in $\mathrm{LN}_{2}$ for a reasonable time ( $\sim 1$ hour) and then taken out and kept at room temperature. The dunking was performed brusquely. In this way the foils were tested in the most extreme conditions. After the thermal cycling tests, the size of the biggest bubbles was measured. The conclusion was that the size and shape of the bubbles was not affected. A picture of the foils after the test is shown in Figure 8.5.

In the second test attempted one single foil was dunked without removing the cover that protects the glue with the layer. As before, the foils were dunked quite bluntly. As seen in Figure 8.6, the foil completely rolled up as soon as it approached the liquid nitrogen. This can be interpreted as the result of the different thermal coefficients of the materials. Indeed, after taking out the foil returned to the usual shape. This behaviour had not happened in the previous test due to the absence of the protective layer and the back-to-back configuration of the foils.

\section{Tests with mesh set-up}

To see whether the thermal coefficient mismatch could be mitigated by placing two DF2000MA foils with the protective cover back-to-back, the foil/mesh combination was then assembled by tightening the screws not maximally to allow for changes in cold. The whole set-up was shaken several times and then the foils were checked by eye looking for scratches. The foil 


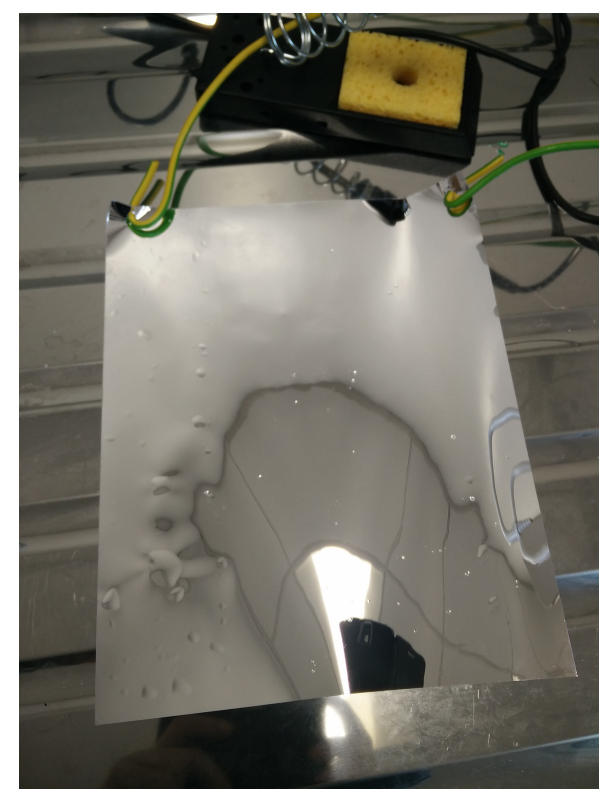

(a) Side A

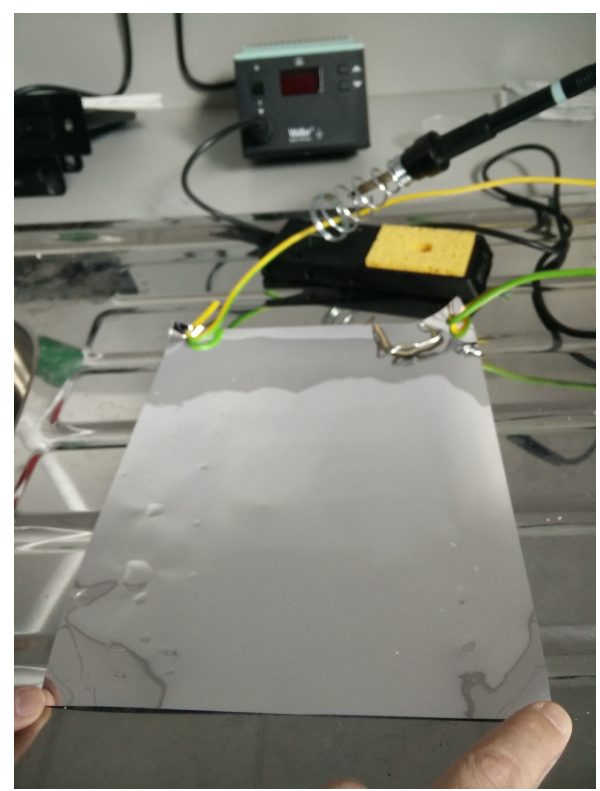

(b) Side B.

Figure 8.5: Two DF2000MA foils glued back-to-back shown after thermal cycling test. In both sides bubbles formed during the glueing are visible.

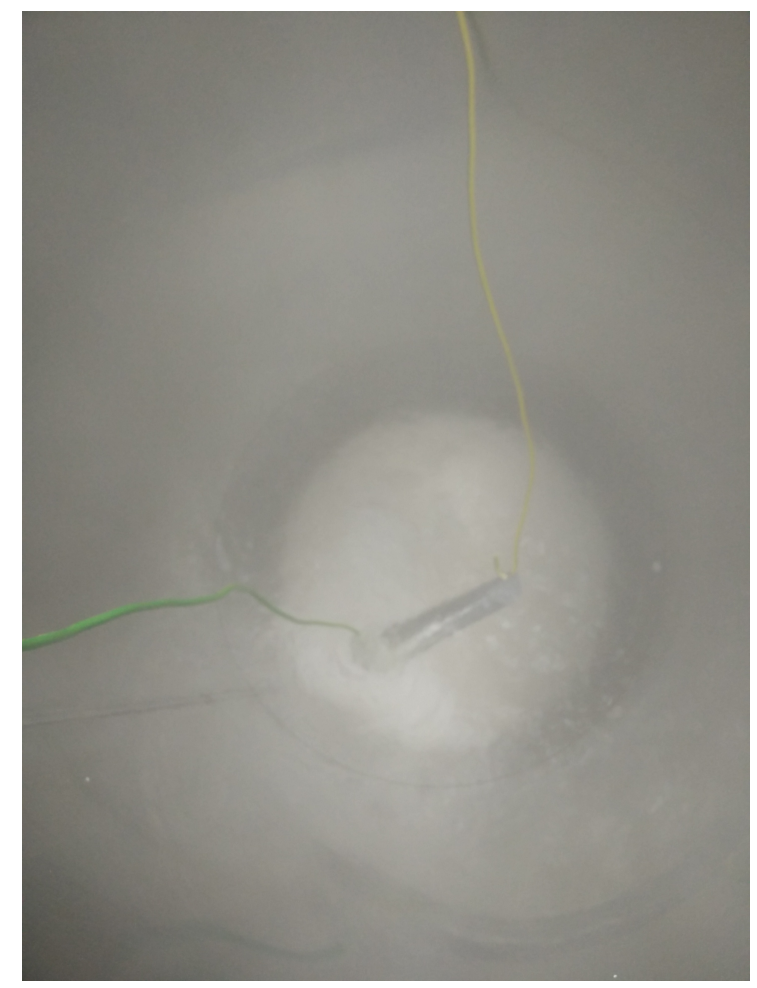

Figure 8.6: Single foil in $\mathrm{LN}_{2}$. Foil is rolled up due to a mismatch of thermal coefficients of the foil and protective cover. 
surfaces looked perfect except for some little dips all around the borders that were entirely covered by the frame.

The set-up was dunked in the $\mathrm{LN}_{2}$ abruptly without waiting for a gradual cooling down. The frame was taken out after about 30 minutes and a pattern of fold was observed in both the surfaces. This is shown in figure 8.7. Folds are clearly visible from the distorted reflection in both sides. This effect was interpreted as the consequence of the pressure applied by

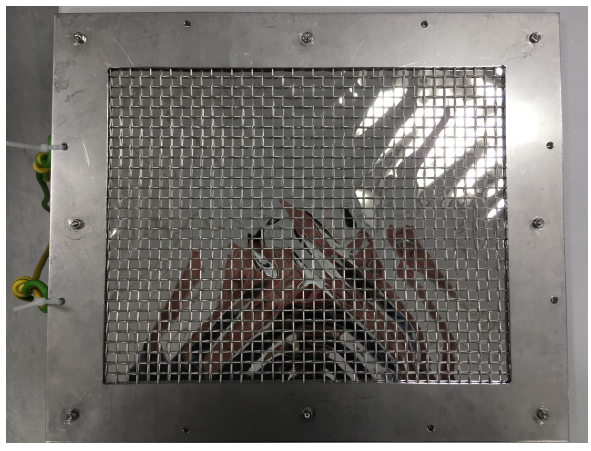

(a) Folds on side A.

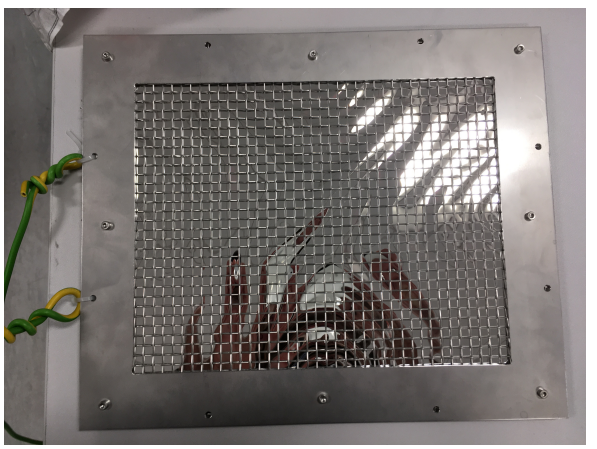

(b) Folds on side B.

Figure 8.7: Folds on foils after cold-test.

the screws. Indeed, the folds seemed to follow a pattern that went from screw to screw. The fact that this appeared only after the cold test was ascribed to the contraction of the materials at low temperatures. One solution tested was to tighten the screws even less as well as attempt a slower and gradual cool-down. Once the screws were slightly loosened, the folds became less accentuated, confirming that the origin was the pressure from the frame in the points were the screws were tight. Apart from that, the foil surfaces were not scratched by the mesh.

The two-half foil set-up was dunked in $\mathrm{LN}_{2}$. Again, dunking and taking out was abrupt. This time there were no folds: the pressure from the screw spread almost uniformly on the surfaces and was strong enough to keep the foils in the position. This showed that the mesh could hold the foils in place.

\section{Laminated foils}

The observed creases on the foils due to the pressure from the screws led to searching for an alternate method that would stiffen the foils. The identified solution was to use lamination on FR4 sheets. DF2000MA foils were laminated on FR4 substrate at the Illinois Institute of Technology (IIT) in Chicago and small prototypes were sent to Manchester for testing in liquid argon. No problems were observed during the dunking tests. 


\subsubsection{Summary of foil tests}

The behaviour of foils at cryogenic temperature was studied in different conditions. Table 8.2 summarises the test results. We have shown that the glue does not affect the purity of liquid argon and therefore we feel confident to use the DF2000MA foils. Thanks to the lamination procedure, the issue of fold on the foil surface was resolved. We have therefore decided to propose the installation of DF2000MA laminated foils to the SBND collaboration.

Table 8.2: Summary of foil tests in liquid nitrogen.

\begin{tabular}{ll}
\hline Test & Note \\
\hline \hline Foil glued b-t-b. & $\begin{array}{l}\text { No problem observed. Size of air bubbles does not } \\
\text { change. }\end{array}$ \\
\hline Single glued foil. & Foil totally rolls up. \\
\hline $\begin{array}{l}\text { Mesh }+ \text { glued foil b-t-b (glue layer not re- } \\
\text { moved). }\end{array}$ & Foldings on surface due to pressure of tight screws. \\
\hline $\begin{array}{l}\text { Mesh+ two halves of glued foil squeezed b- } \\
\text { t-b (glue layer not removed) }\end{array}$ & No problem observed. \\
\hline Laminated foils. & No problem observed. Final solution. \\
\hline
\end{tabular}

\subsection{Low Temperature Evaporation (LTE)}

The foils have to be covered with a WLS compound - TPB. The deposition is performed through a low temperature evaporation (LTE), performed in a vacuum chamber equipped with one or more crucibles in the bottom called evaporator. The substrate is attached on a rotating disk hung at the top of the chamber, facing towards the crucibles at the bottom. The general procedure consists of preparing the WLS compound in one or more crucibles, pumping the vacuum in the chamber and heating the crucible(s). When the temperature is high enough, the WLS compound starts to evaporate. During the evaporation, the substrate is exposed to the evaporating WLS substance, which attaches to its surface. The rotating disk assures a uniform deposition. It is important that the procedure is carried on in a low pressure environment, in order to avoid contaminations during the coating. A deposition sensor placed inside the chamber is used to monitor the evaporation showing the rate of deposition and the deposition thickness. When all of the WLS compound evaporates, gas nitrogen is injected into the chamber to break the vacuum. It is necessary to use dry gas nitrogen (or argon) to avoid humidity normally contained in air that could deteriorate the evaporated layer. 


\subsection{Setting up the large-scale evaporator}

The size of the SBND cathode requires the size of an evaporator larger than most previously used, for example in the WArP experiment [61]. The chamber described in this chapter was custom built and is able to perform large size evaporations. The evaporator was placed in the HEP group Clean Room in the Schuster Building. A clean room is necessary to avoid contamination of impurities in the chamber during the evaporation. The chamber was installed, set up, tested and used for test evaporations which were used in the LArIAT detector.

\subsubsection{Installation}

The evaporator is a cylindrical vacuum-chamber of $90 \mathrm{~cm}$ diameter closed by a lid on top (Figure 8.8) and can evaporate $60 \mathrm{~cm} \times 60 \mathrm{~cm}$ substrates. It has three CF40 flanges on the

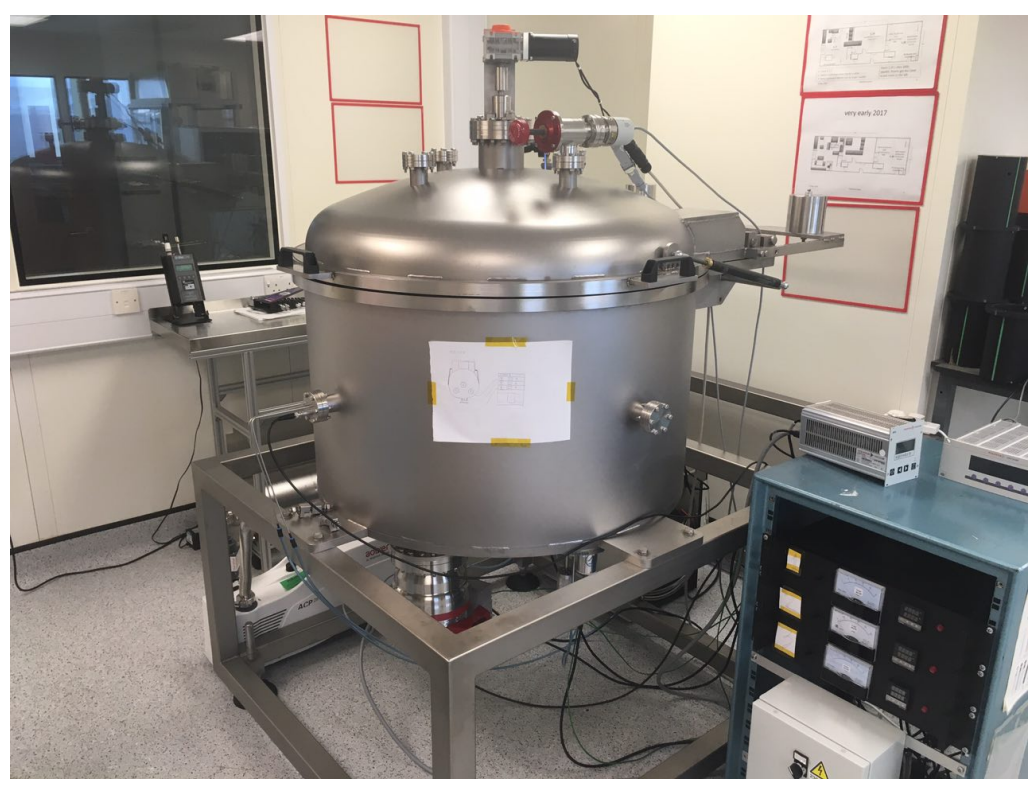

Figure 8.8: Photo of the evaporator installed in the clean room.

top, three CF40 on the sides plus 6 CF63 flanges on the bottom and a CF100 flange for the vacuum pump. The main components of the evaporator are listed below.

- Three crucible systems to be installed on the bottom of the chamber. Each of them has its own power supply for the heating circuit. They are connected in series to a chiller which provides cooling using cold water. A photo is visible in Figure 8.9.

- Pumping station: a PFEIFFER VACUUM vacuum pump ACP 28 used to draw air from the chamber up to $\sim 10^{-2}$ mbar and a turbo pump PFEIFFER VACUUM HiPace $700 \mathrm{M}$, necessary to reach low values of pressure $\sim 10^{-6}$ mbar. 


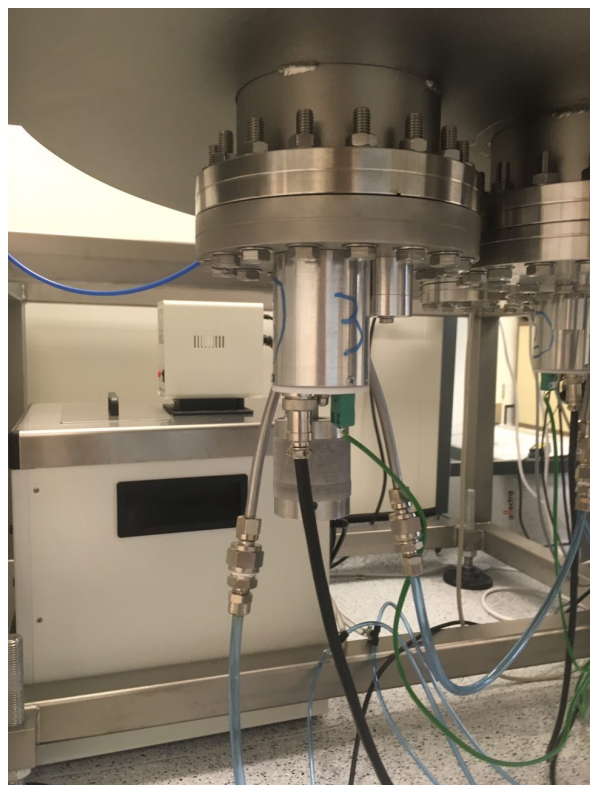

Figure 8.9: Photo of one crucible system installed on the bottom of the evaporator. The black cable comes for the power supply and the green wire is for the readout of the temperature. Cold water is pumped through the blue tubes.

- A metallic disk to be hung on the lid of the chamber (Figure 8.10(a)). An electric motor situated on the top of the lid controls the rotation of the disk.

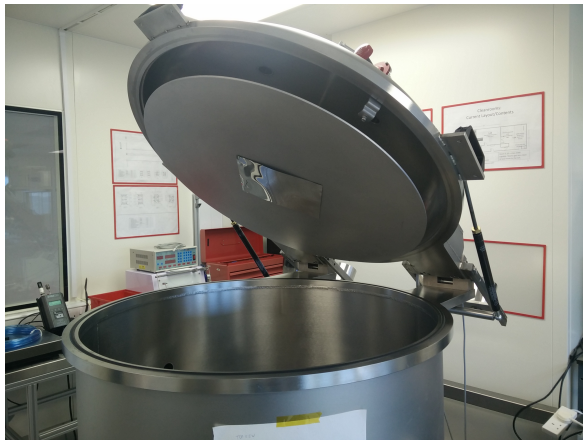

(a) Before the evaporation.

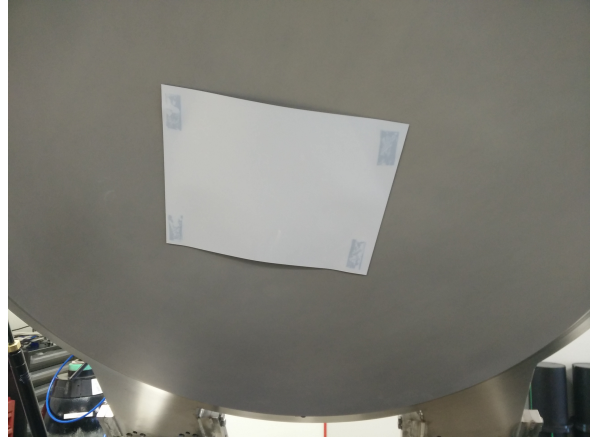

(b) After the evaporation.

Figure 8.10: Foil installed on rotating disk hung on the evaporator lid before and after the evaporation.

- Deposition sensor. The deposition sensor is a metallic shaft that ends with a $14 \mathrm{~mm}$ gold crystal. It was mounted from the side of the chamber, facing the bottom, i.e. the evaporation source. The sensor was connected through a flange to the outside and was read out by means of a BNC cable connected to a converter box and a laptop.

- Gas nitrogen bottle to break the vacuum once the evaporation is finished.

- Pressure sensor PFEIFFER VACUUM Compact Full Range Gauge PKR 251, installed 
through a screw valve on the top of the evaporator lid. The valve was kept closed during the actual evaporation to prevent the sensor to be coated with TPB. The pressure was read and displayed by a PFEIFFER VACUUM vacuum measurement and control unit MaxiGauge TPG 256 A.

The crucible systems are effusion cells developed specifically for vacuum systems and have a capacity of $15 \mathrm{~cm}^{3}$. Aluminium cylinders that fit in the copper body of the effusion cell can be easily removed which facilitates insertion of TPB. The cells have a rotating shutter that is manually operated from the outer side on the bottom of the chamber through a magnetic translator (depicted in Figure 8.9). Every cell has a copper lid with a $4 \mathrm{~mm}$ hole in the centre used to collimate the flux of the evaporating TPB.

A photo of the inside of the chamber after the installation of every component is shown in Figure 8.11. The photo shows the deposition sensor (rod on the left) and the three effusion cells installed with the rotating shutter closed. Three of the six CF63 flanges were not exploited. The hole for the turbo pump is also shown (left).

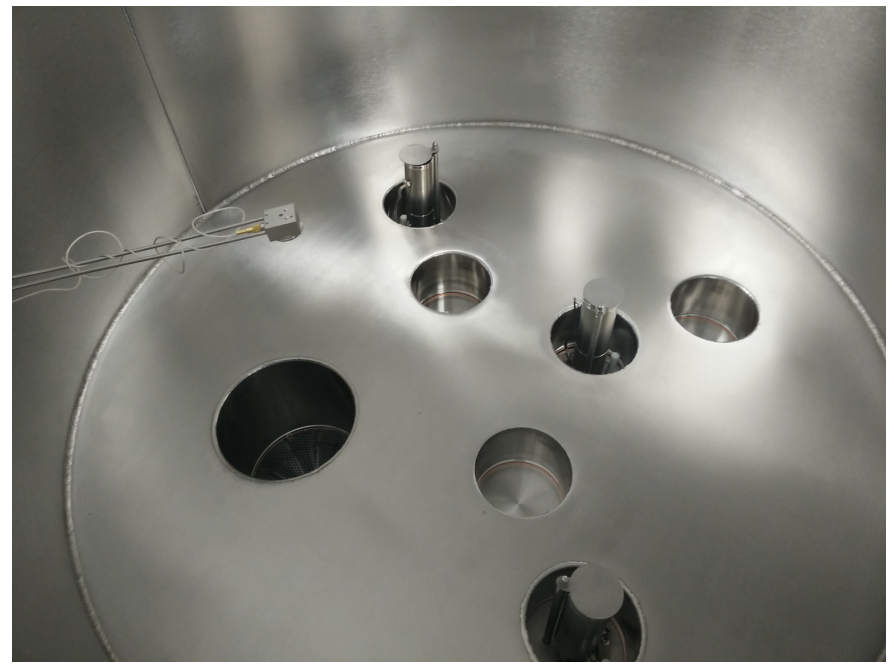

Figure 8.11: Inside bottom view of the evaporator. Three crucible cells with shutters, the pump outlet (largest hole) and the deposition sensor (rod on the left) are shown.

\subsubsection{Evaporation procedure}

In order to assure a repeatable outcome of evaporations, a procedure of evaporation was developed based on one created for the WArP experiment at Laboratori Nazionali del Gran Sasso (LNGS). The evaporation procedure can be summarised in the following steps.

1. Prepare foil of the desired size.

2. Install the foil on the rotating disk. 
3. Weigh the TPB. The TPB was in form of a white powder and was weighed using a precision scale.

4. Deposit the TPB in the aluminium crucible, insert it in the effusion cell and install the copper lid. Tests described here were performed using the crucible located at $15 \mathrm{~cm}$ from the centre (central in Figure 8.11). Close the shutter using the outer knob.

5. Append rotating disk on the evaporator lid.

6. Remove protective film from the foil.

7. Close the chamber and tighten the main flange with two holders to close.

8. Turn on the pump. Once the pressure is $5 \times 10^{-1}$ mbar turn on the turbo pump and wait until $10^{-6}$ mbar.

9. At $10^{-5}$ mbar turn on the chiller at $20^{\circ} \mathrm{C}$ in order to start pumping cold water in the crucibles and the deposition sensor.

10. When the pressure reaches $10^{-6}$ mbar, close the pressure sensor valve and turn on the crucible heater by switching on the power supply.

11. When crucible temperature reaches $200{ }^{\circ} \mathrm{C}$ turn on the deposition sensor and rotating motor at speed $4 / 5$.

12. When crucible temperature reaches $220^{\circ} \mathrm{C}$ open the crucible shutter.

13. At the end of the deposition, close the shutter, turn off crucible heater, deposition sensor and rotating motor.

14. Turn off turbo and vacuum pump. Break vacuum with gas nitrogen once pump has stopped rotating.

15. Open the chamber, remove disk and remove the evaporated foil.

16. Turn off the chiller.

During the procedure, the sensor was used to understand the status of the evaporation. A plot of the sensor data is shown in Figure 8.12. The sensor was not calibrated at the time, so it was not possible to have information on the absolute deposition of TPB. However, it was still a useful tool to understand when the evaporation started and when all the TPB evaporated. From the plot it is easy to identify the beginning of the evaporation (at $\sim 400 \mathrm{~s}$ ), 
the rising of the thickness as well as the rate, and the flattening of the thickness corresponding to the dropping of the rate when all the TPC has evaporated at $\sim 680 \mathrm{~s}$.

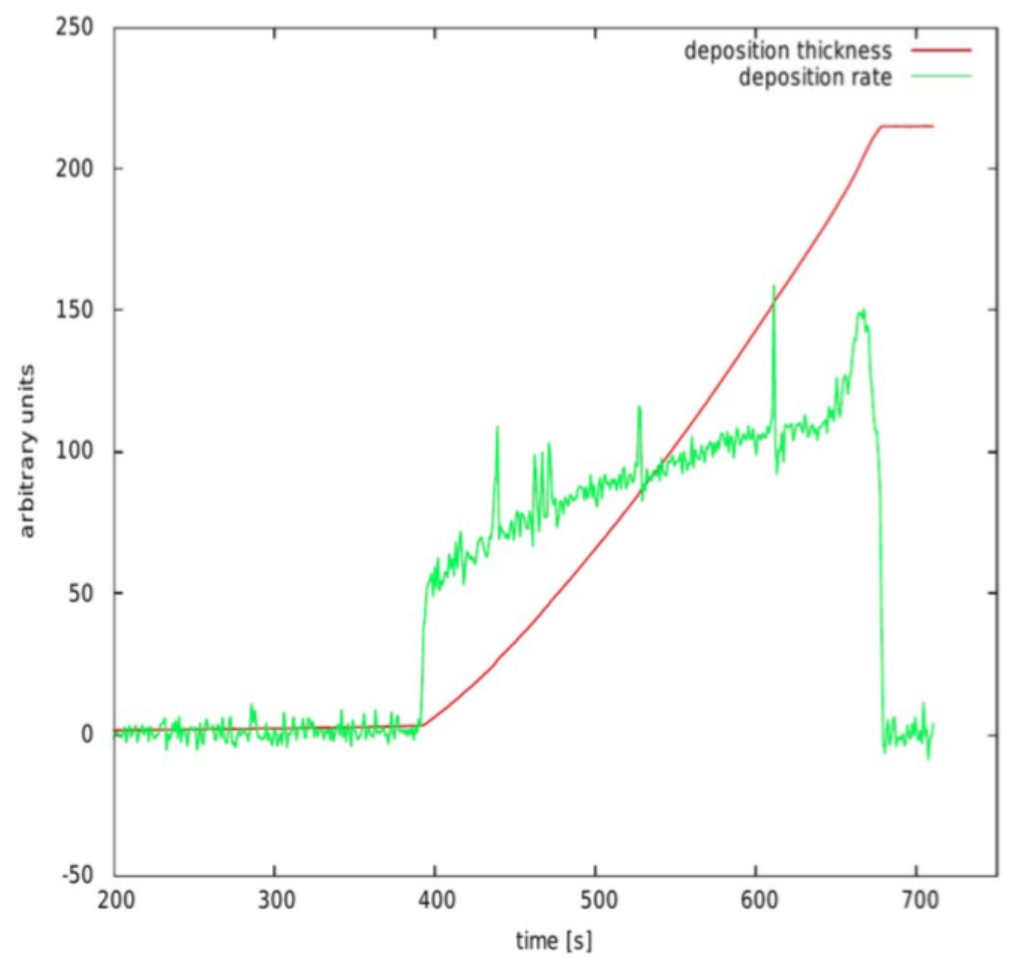

Figure 8.12: Data from the evaporation sensor. Red curve: deposition thickness as a function of time. Green curve: deposition rate.

\subsubsection{First evaporation and dunking test}

The first tests were performed with a small VIKUITY foil. The main purpose was to test the device and optimise the procedure. The size was chosen such that it could also be possible to test the evaporated foil in liquid nitrogen $\left(\mathrm{LN}_{2}\right)$. The set-up before the evaporation is shown in Figure 8.10(a) while Figure 8.10(b) shows the set-up after the evaporation. The evaporated test foil was then squeezed in the SBND-like mesh cathode and dunked in liquid nitrogen (Figure 8.13). After the test, the foil was removed from the frame and the surface was inspected. A photo is conveyed in Figure 8.14. We noticed then the surface was scratched in some points, probably as a consequence of the contraction of the metallic mesh at cryogenic temperatures. Nevertheless, the scratched zones were fully covered by the frame and mesh meaning that the evaporation layer on the exposed surface can resist at cryogenic temperatures. The procedure was successful and we also showed that the mesh does not damage the exposed surface of the evaporated foil from the point of view of operation inside the detector. We therefore proceeded to evaporate foils to be installed in the LArIAT 


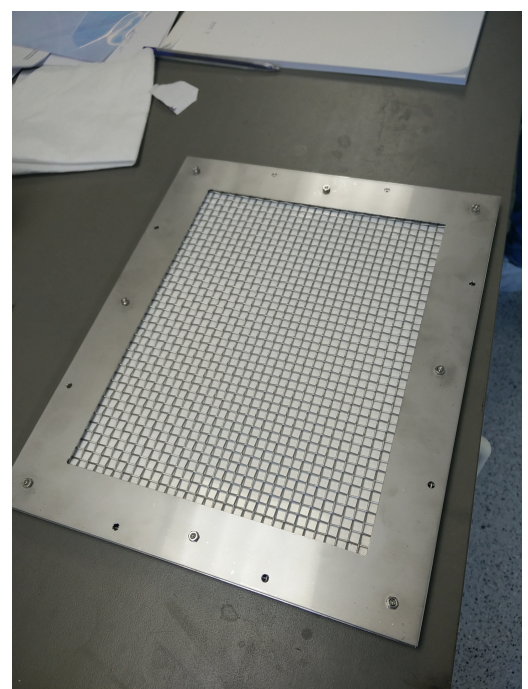

(a)

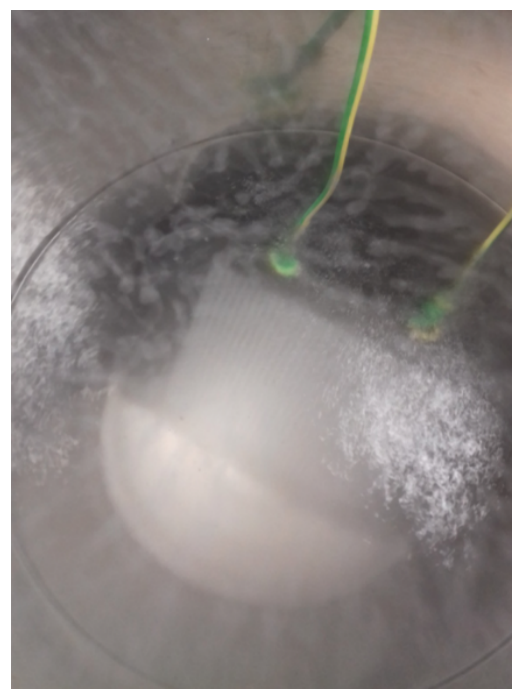

(b)

Figure 8.13: Test of the evaporated foil. Left: foil squeezed in a SBND-like mesh cathode. Right: mesh/foil set-up dunked in liquid nitrogen.

cathode.

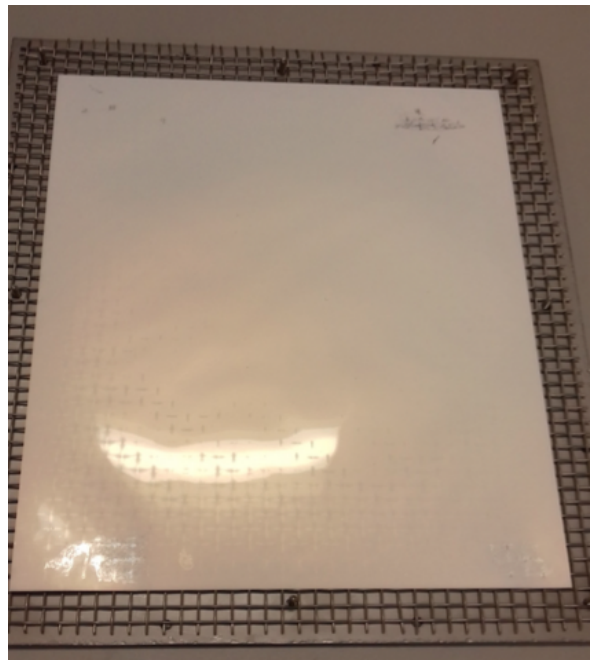

Figure 8.14: Evaporated foil after the dunk test in liquid nitrogen. There is an effect of the mesh frame that scratches the TPB layer. However, the flawed areas were entirely covered by the frame.

\subsubsection{Evaporator calibration}

The thickness of TPB deposition is a function of the amount of TPB and the position of the crucible in the chamber. A basic simulation developed for the Gran Sasso evaporator was employed to understand the amount of TPB to prepare in the crucible to obtain a deposition of about $300 \mu \mathrm{g} / \mathrm{cm}^{2}$. This value was chosen based on the study of TPB optical properties 
developed in [62]. The simulation allowed to account for the geometry of the chamber, the position of the crucible (in our case $15 \mathrm{~cm}$ or $30 \mathrm{~cm}$ from the centre) and the position disk in the chamber. Figure 8.15 presents the plot obtained from the simulation. The final decision

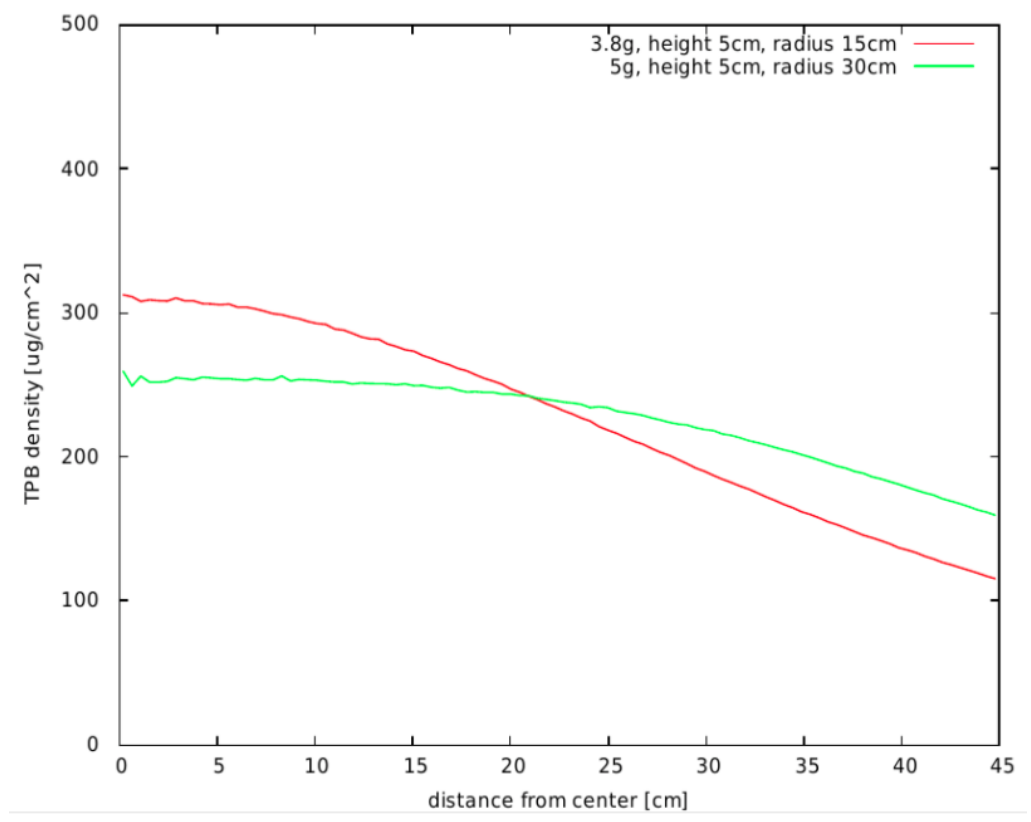

Figure 8.15: TPB density as a function of the distance from centre of the rotating disk. Red curve: simulation for $3.8 \mathrm{~g}$ of TPB and crucible at $15 \mathrm{~cm}$ from the centre of the chamber. Green curve: simulation for $5 \mathrm{~g}$ of TPB and crucible at $30 \mathrm{~cm}$ from the centre of the chamber.

was to use the crucible at $15 \mathrm{~cm}$ from the centre and $3.8 \mathrm{~g}$ of TPB. This configuration allows to have a deposition density closer to the desired $300 \mu \mathrm{g} / \mathrm{cm}^{2}$ up to $\sim 20 \mathrm{~cm}$ from the centre of the chamber.

It is important to understand the reliability of the simulation and therefore dedicated calibration runs of the evaporation were performed. Small pieces of di-electric foils were placed in defined position on the rotating disk. Each of them was previously weighted with a precision scale after removing the covers. In the weighting procedure, the scale was shielded with a plastic cover to avoid air drafts and the set-up was wrapped in aluminium foils to shield from electrostatic disturbances. The weighting procedure was repeated after the evaporation of the foils. The TPB deposition was estimated as the difference in weight between the before and after measurement. The different position of the different foil pieces allows to map the deposition as a function of the radius in the chamber. The results are shown in Figure 8.16 which shows the deposition thickness as a function of the distance from the chamber centre. These preliminary results led us to believe that the obtained deposition thickness is compatible with the simulation. 


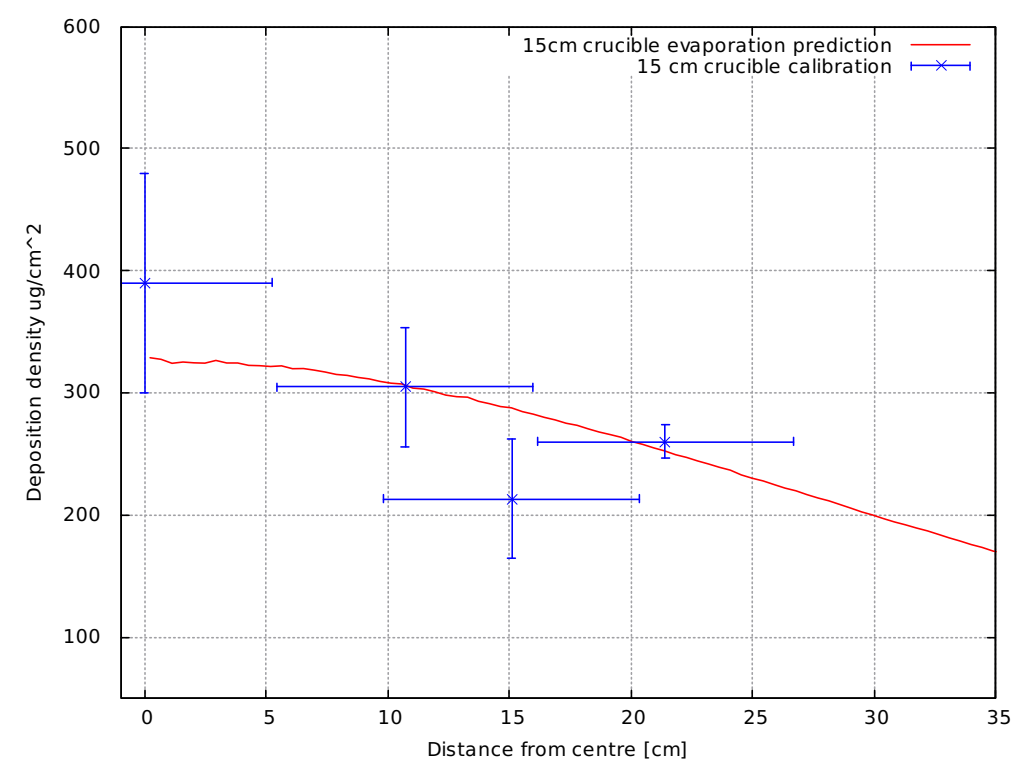

Figure 8.16: Deposition thickness vs. distance from the centre. The red line is the prediction of the simulation while the blue points are the results of the measurements.

\subsection{Foils and mesh cathode in LArIAT}

A final test before proposing the installation of WLS foils in the SBND cathode was to test its performance in a running LArTPC.

\subsubsection{Mesh cathode}

An SBND-like cathode plane assembly was constructed in the university workshop based on the design of the SBND CPA step files (see Figure 8.17).

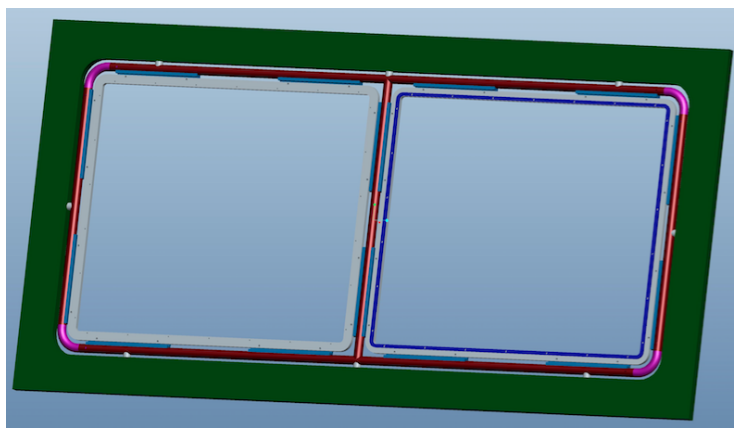

Figure 8.17: Design of the SBND-like cathode installed in the LArIAT detector.

The cathode is a two window frame made from stainless steel which holds two layers of mesh, necessary to guarantee the transparency. The border of the frame is fixed to a $10 \mathrm{~cm}$ G10 frame, needed to isolate the cathode from the stainless-steel cryostat which is at ground. The cathode is fixed to the TPC frame using an epoxy glue. It was manufactured in Manchester 
and shipped to Fermilab to be installed in the LArIAT detector (see Figure 8.18).
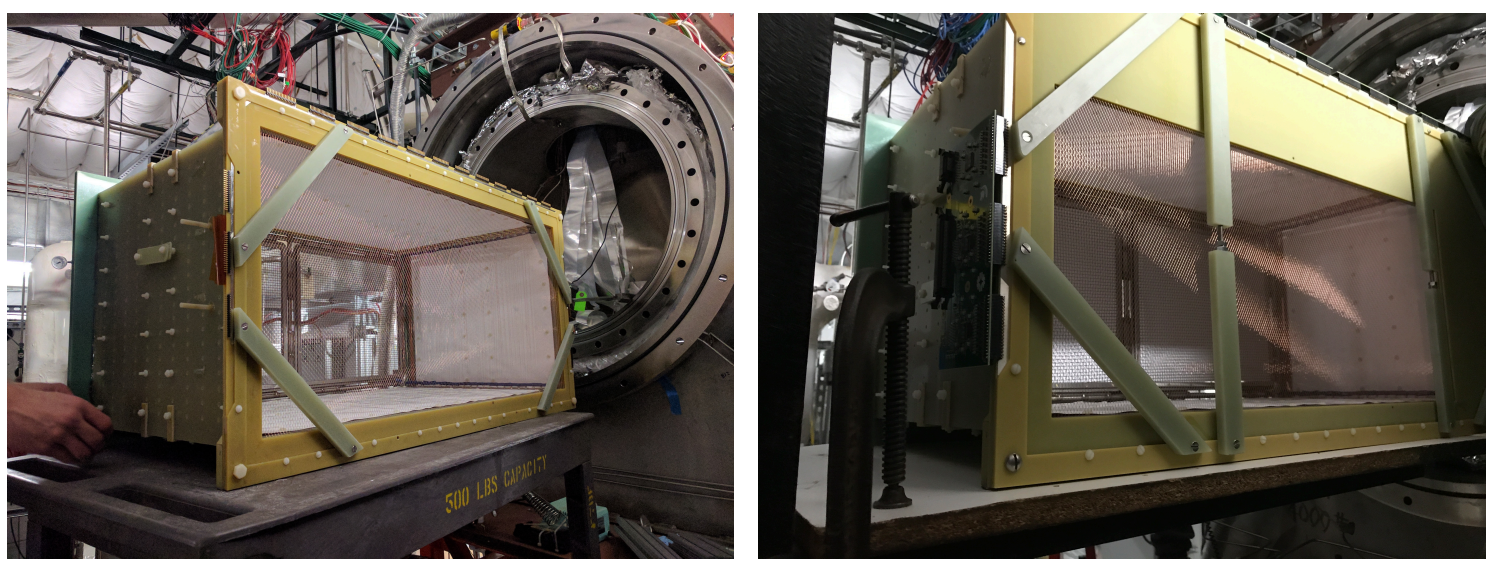

(a) TPC during Run IIIa: mesh cathode installed with-(b) TPC during Run IIIb: mesh cathode plus reflector out reflector foils. foils.

Figure 8.18: The LArIAT TPC with the mesh cathode installed.

\subsubsection{LArIAT run with evaporated reflector foils}

Given the size of the LArIAT detector, 2 VIKUITY foils of $33.5 \mathrm{~cm} \times 39.5 \mathrm{~cm}$ were prepared. The amount of TPB used was estimated using the dedicated simulation. The evaporated foils were then wrapped in plastic and shipped to Fermilab.

During the first part of Run III the cathode was installed without foils placed between the mesh. The evaporated foils were added for the second part of the run by sandwiching the foils between the two mesh layers. A close-up of the foils squeezed between the mesh is conveyed in Figure 8.19.

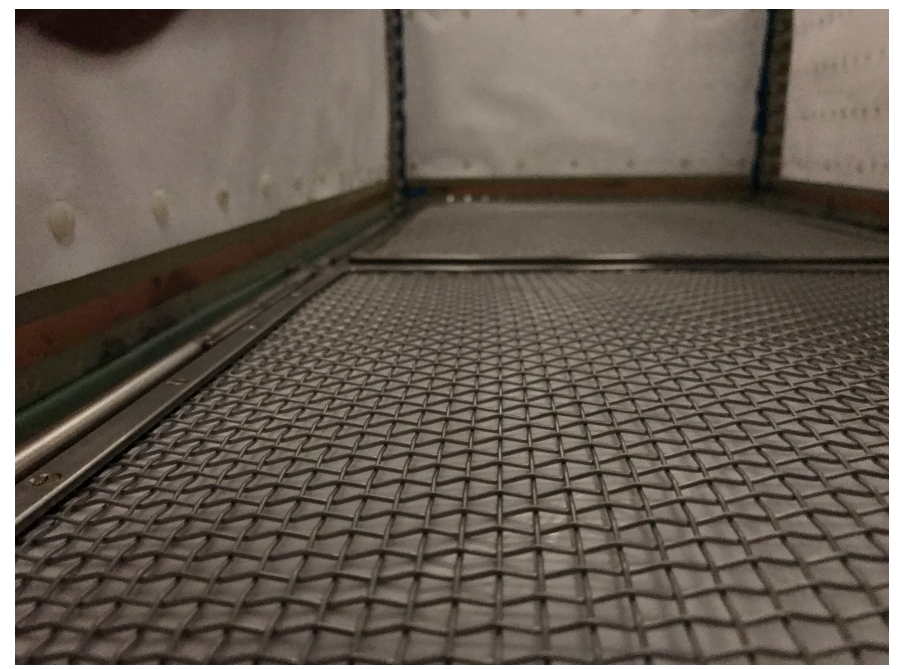

Figure 8.19: The LArIAT cathode with foils installed between layers of mesh. 
The data collected showed that the mesh cathode and the foils did not compromise the behaviour of the detector. An improvement of light yield of the order of $20 \%$ was observed. This is not a large improvement, but it conforms to expectations when taking into account that the fieldcage was already covered by foils. Other modifications were implemented in the detector between Run IIIa and Run IIIb, that could also affect the light yield. The wire pitch in Run IIIa was $5 \mathrm{~mm}$ which has been modified to $3 \mathrm{~mm}$ in Run IIIb meaning that less light was able to reach the two PMTs installed behind the APAs. Also, the modified APAs required additional G10 installation (see Figure 8.18(b) compared to Figure 8.18(a)) which reduced the light collection. More precise studies are needed with the updated detector geometry to understand the full impact of the detector modifications.

\subsection{Conclusion}

In this chapter we have presented the study to develop the methods and procedures to install WLS covered reflector foils in a mesh cathode. We have tested different types of foils in terms of mounting schemes and verified that the presence of foils does not affect the purity of liquid argon. The combination of DF2000MA laminated on FR4 has been identified as the best solution in terms of stability in liquid nitrogen.

A large-scale evaporator has been installed and the evaporation procedure has been defined and tested. A calibration has also been performed in order to validate the simulation used to predict the deposition density.

The evaporated foils have been installed in LArIAT, a test-beam experiment, and successfully operated for one month. All of the above results were used in a proposal to install foils which has just been submitted to the SBND technical board. 


\section{Chapter 9}

\section{Beam Shape Studies for Delayed}

\section{Ion Modelling}

\subsection{Introduction}

The MicroBooNE experiment [34] has seen an unexpected excess of scintillation light. One of the possible explanations could be the presence of positive ions, left over from ionisation events (see Figure 4.3), that recombine while drifting towards the cathode and emit light. The positive ions would drift very slowly which means that the scintillation light can not be correlated to the TPC interaction that generated the positive ions and the effect would be similar to random noise. This, particularly in case of the ions recombining at the cathode, could have a large impact in the SBND detector, should the cathode be covered with reflector foils, since it would lead to high rate of extra photons in the PMTs.

A dedicated set of runs was taken with the LArIAT detector to understand whether this excess scintillation light is present and how it depends on charge deposited in the TPC. A correlation was found between rate of light emission and the amount of charge deposited in the detector. A study was performed to understand whether some simple models for late light resulting from positive ion recombination could explain the pattern of the late light observed in LArIAT. The ion drift velocity is of the order of $v_{\text {ions }}=0.8 \mathrm{~cm} / \mathrm{s}$, meaning that it would take around one minute for the ions to drift the entire width of the LArIAT TPC, which happens to coincide with the time between beam spills (see Figure 9.1 for a scheme of the beam time structure). LArIAT is therefore the ideal venue to test the hypothesis of delayed light coming from recombining ions since the light that would come from ion recombination does not overlap with the light from beam particle interactions. 


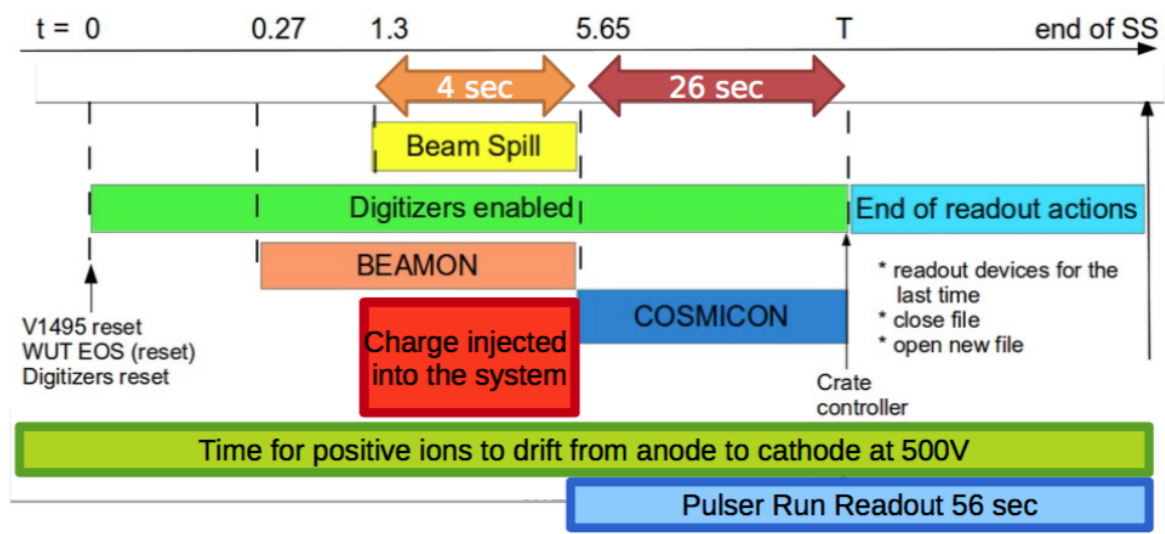

Figure 9.1: LArIAT beam structure. The time duration of the beam spill, the readout and the time for positive ions to drift from anode to cathode are highlighted.

\subsection{LArIAT data runs}

It is expected that random scintillation light resulting from recombining ions should increase with the electric field as a smaller number of ions recombine immediately after ionisation (the higher the electric field, the stronger is the force pulling the charges away from the interaction region). Opposite behaviour is expected from standard scintillation light coming from recombination at the interaction point (see Figure 4.4).

A dedicated set of runs was taken to search for random light at different electric field values and different beam intensities with the LArIAT detector, to test the hypothesis of scintillation light due to ion recombination. As the light was expected to be similar to random noise, a pulser trigger was used to acquire waveforms out of time with scintillation events. To test the dependency of the light with respect to the electric field, data of single photo-electron rates was taken in July 2016. The data-set consisted of cosmic ray runs taken in the $0-700 \mathrm{~V} / \mathrm{cm}$ electric field range and with beam at different intensities $\left(10^{9}, 10^{10}\right.$ and $7 \times 10^{10} \mathrm{PPP}^{1}$ ) at 0,300 and $500 \mathrm{~V} / \mathrm{cm}$. Similar data was taken in 2017 with the mesh cathode installed with and without foils, but this data-set has not been analysed yet.

A clear effect of the beam intensity in terms of scintillation light was observed, i.e. more light was collected at higher intensities and the measured light noise was found compatible with a superposition of two components: random distribution and liquid argon scintillation light. One data-set is shown in Figure 9.2 [63], where, for a $10^{10} \mathrm{PPP}$ intensity and $500 \mathrm{~V} / \mathrm{cm}$ electric field, a clear excess above the no-beam data can be observed.

\footnotetext{
${ }^{1}$ Protons per pulse.
} 
PE rate

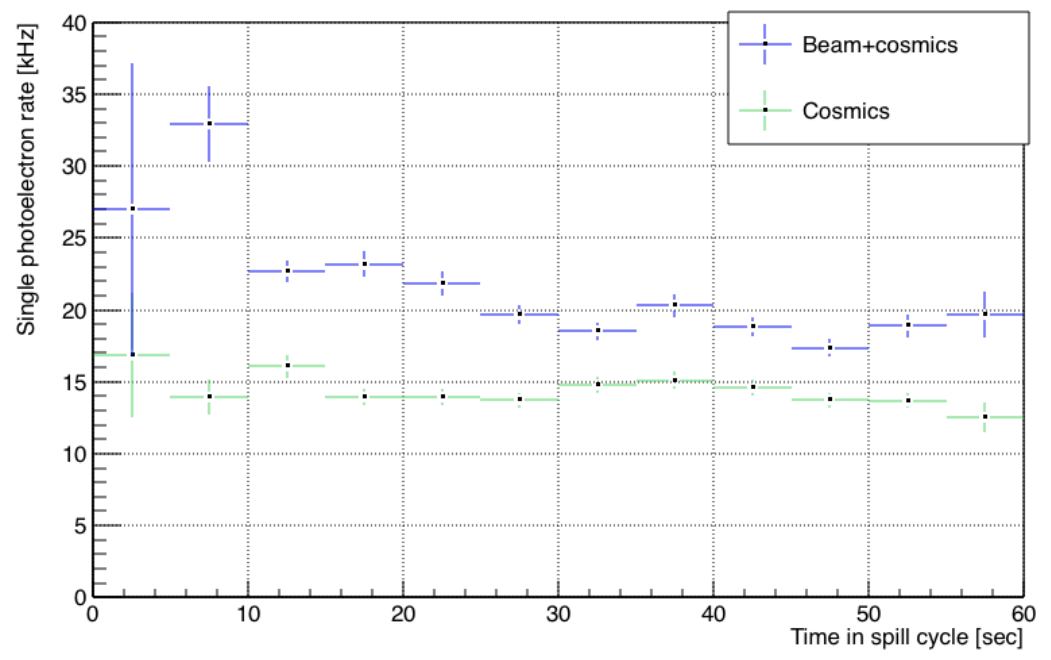

Figure 9.2: Data of single photoelectron rates taken by LArIAT in July 2016 [63]. The plot shows the histogram for data of beam+cosmic rays (blue points) and only cosmic rays (green points).

\subsection{Simulation procedure}

To better understand the situation, a simulation was developed and compared with a subset of the data taken at $500 \mathrm{~V} / \mathrm{cm}$ of cosmic rays and beam. We used a simulation of beam and cosmic rays in order to predict the distribution of energy deposition in the TPC active volume. The core part of the simulation was developed in LArSoft, a physics simulation software for LArTPCs [64]. The energy deposition shape was propagated using a simple drift model to transform it into a distribution of scintillation events in time and compared with time distribution of the data obtained with the detector.

\subsubsection{Beam simulation}

The beam simulation begins with the LArIAT secondary beam propagating 10 spills with $300,00064 \mathrm{GeV}$ pions and with the bending magnets set at $100 \mathrm{~A}$. The LArIAT beam line was modelled using G4beamline [65], a GEANT4 scripting tool for particle simulations in beam lines. The script returned as output a TTree containing information about each particle (PDG number, momenta, coordinates, etc.) at each detector in the beam line. A Fermilab Hierarchical Configuration Language (FHiCL) [66] script and a dedicated module to translate the G4Beamline results into LArSoft module called "LArIATFileGen" were then used to select a trigger condition and to propagate the particles in the TPC. Once a particle had satisfied the trigger condition, a time window of $\pm 393 \mu$ s was opened and each particle 
that happened to be in the TPC volume in that time-frame was simulated in LArSoft. This procedure simulates the behaviour of the LArIAT detector, where additional particles that do not cause the trigger can enter into the drift region during readout. The imposed trigger condition (see Figure 5.6 for a reference) was TOF 1 \& MWPC1 \& MWPC2 \& MWPC3 and only the particles passing through the collimator were accepted allowing for 60-70 triggering particles per spill on average. This trigger configuration was set following the assumption that the collimator in beam line will stop most other particles. Information about deposited energy and relative position was obtained storing the information provided by the class sim: : IDE in LArSoft. This class provides the true energy as "read" from the TPC wires. The predicted energy depositions in the TPC are shown in Figure 9.3.

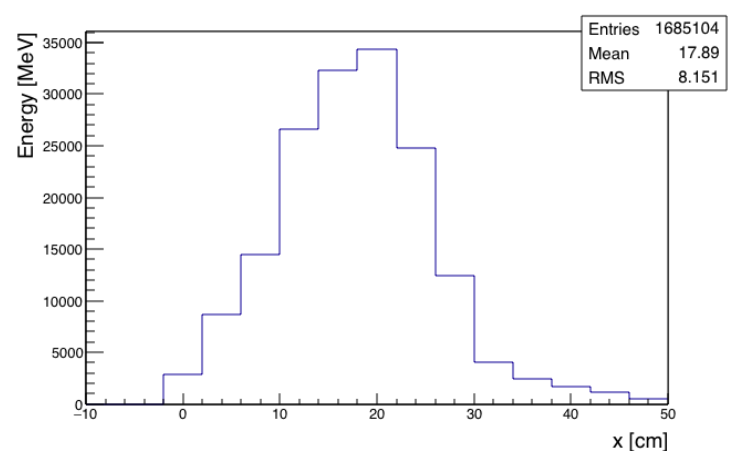

(a) Shape of deposited energy along the $x$-axis.

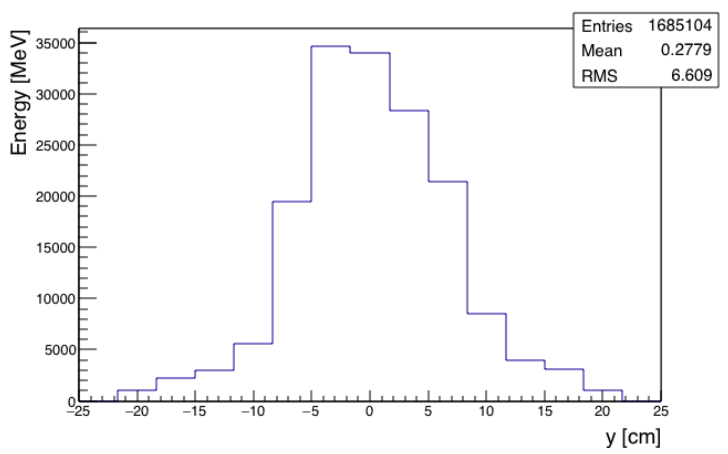

(b) Shape of deposited energy along the $y$-axis.

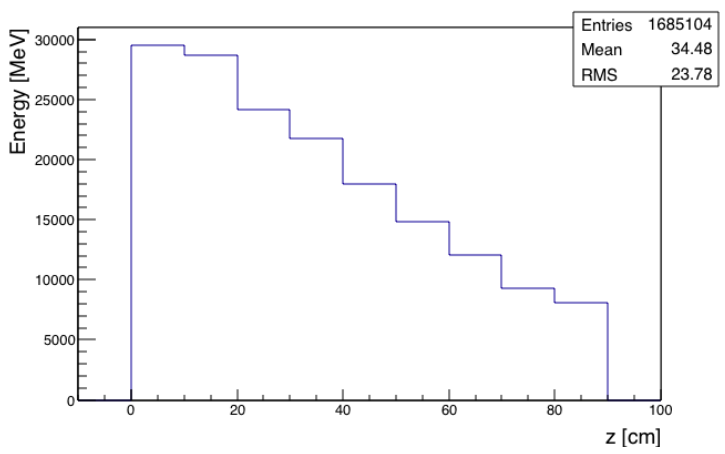

(c) Shape of deposited energy along the $z$-axis.

Figure 9.3: Deposited energy distribution in $x, y, z$ adding the contribution of the ten spills simulated.

\subsubsection{Cosmic ray simulation}

The cosmic ray simulations was implemented using CORSIKA [67], a program dedicated to the simulation of particle showers originated from cosmic ray interactions in air. In particular, a number of events equivalent to 20-minute exposure of the detector to cosmic 
rays was simulated. This data set enabled a data-driven modelling of the background in the beam plus cosmic rays data sample. Since CORSIKA simulates a realistic cosmic ray flux, it was possible to use the energy deposition of simulated cosmic rays in the TPC to infer the conversion constant between energy deposition $(\mathrm{MeV})$ and single photoelectron rate $(\mathrm{kHz})$. For this purpose the rate obtained during a cosmic-only run (green points of Figure 9.2) was used. This is an approximate model that does not take into account the optical acceptance of the light collection system and inefficient factors.

\subsubsection{Comparison with data}

The distribution of the total (beam+cosmic rays) energy deposition was propagated towards the cathode with a velocity equal to that of the drifting ions, also assuming a dispersion of $\sigma_{1}=0.1 \mathrm{~cm} / \mathrm{s}$ and $\sigma_{2}=0.3 \mathrm{~cm} / \mathrm{s}$. The results, overlaid with the data of a cosmic rays plus beam run, is shown in Figure 9.4.

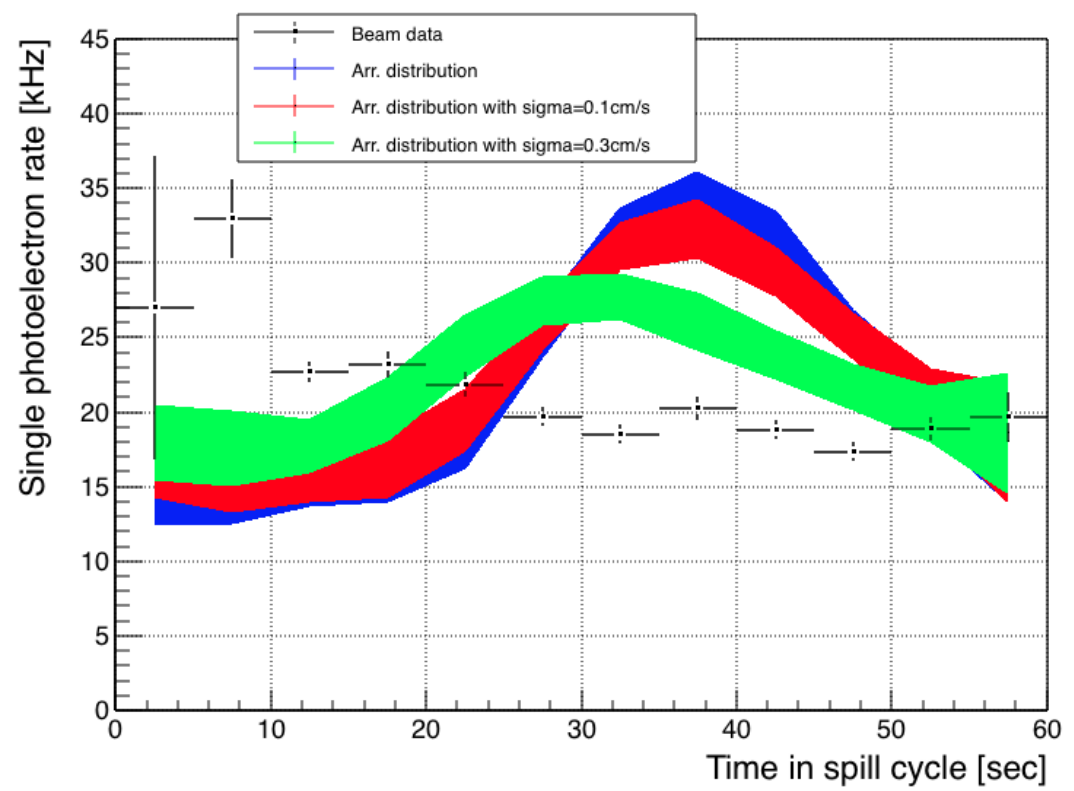

Figure 9.4: Distribution of ion arrival time at the cathode. The plots shows beam plus cosmic rays data (black points), arrival time for ions drifting at $v_{\text {ions }}$ (blue curve) with $0.1 \mathrm{~cm} / \mathrm{s}$ (black line) and $0.3 \mathrm{~cm} / \mathrm{s}$ (green line). The width of colour bands is an estimation of the statistical error on the Monte Carlo simulation.

The beam simulation was then normalised with respect to the data surplus observed in the beam-plus-cosmic data acquisition. The simulation suggests that the observed light pattern does not match the hypothesis of ions recombining at the cathode. However, further studies need to be performed to fully understand the systematics and detector effects. 


\subsection{Conclusion}

We have developed a simple model to test the hypothesis of positive ion recombination as an explanation for delayed ion light. A shape-only study seems to indicate that recombination at the cathode is unlikely, however further analysis with full accounting of errors and systematics would be needed to exclude it. Further simulations studying the exponential decay of ions during the drifting are currently ongoing. 


\section{Chapter 10}

\section{Conclusions}

Liquid argon TPCs are detectors that offer precise reconstruction of neutrino interactions using charge collected by a great number of wires and scintillation light. The challenging future measurements in neutrino physics will require large-scale experiments which present new challenges in construction and design. The work exhibited in this dissertation falls within the framework of research and development for these detectors.

A new electric technique to measure wire tension has been developed, that can be used in LArTPC detectors such as SBND. We demonstrated that about $90 \%$ of the SBND wire segments can be measured with a resolution of a few grams and that the tension is determined with a bias smaller than $20 \mathrm{~g}$ for the typical length of a segment in SBND, well within the tolerance of $100 \mathrm{~g}$. The technique allows to perform measurements in situations in which it would be much more difficult or impossible with the currently used laser technique. For the first time it has been possible to monitor the change in tension of Copper-Beryllium wires during a cool down to cryogenic temperature, observing an effect of the different contraction of the frame materials leading to an increase of the tension of $82 \mathrm{~g}$. We performed a dedicated study of different ways to fix wires to the electronic boards testing soldering and glueing. The test showed that there is no preference in terms of tension loss among different methods in the short term period. Finally, we demonstrated the feasibility of applying the method to simultaneously measure the tension of up to 32 wires and tested on a SBND mock-up APA at Daresbury Laboratory.

Simulations show that TPB-coated reflector foils on the cathode can potentially enhance LArTPC position and timing resolution. Studies and tests have been performed to develop the methods and infrastructure to install WLS-covered reflector foils inside the SBND detector. This included the selecting of the type of foils that best fit the installation requirements and the benchmarking of the TPB-evaporation procedure. The evaporated foils 
were installed in a SBND-like mesh cathode mounted in LArIAT, a test-beam experiment at Fermilab, and run successfully.

A simple model to test positive ion recombination has been developed. It showed that recombination is unlikely to happen at the detector cathode, but further investigations and more detailed simulations are needed to determine the mechanism of delayed light emission.

The electric wire tensioning technique passed a readiness review of the SBND collaboration and an instrumental paper describing it is in preparation. The results of the test in LArIAT of the evaporated foils were in the writing of a formal proposal to install evaporated foils in the SBND detector that has been presented to the collaboration and is currently under evaluation. Finally, the ion studies, an important aspect of understanding the behaviour of scintillation light in large-size argon detector, can potentially lead to a publication down the line. 


\section{Bibliography}

[1] S. Schael et al. [ALEPH and DELPHI and L3 and OPAL and SLD Collaborations and LEP Electroweak Working Group and SLD Electroweak Group and SLD Heavy Flavour Group], Phys. Rept. 427 (2006) 257 doi:10.1016/j.physrep.2005.12.006 [hepex/0509008].

[2] Y. Fukuda et al. [Super-Kamiokande Collaboration], Phys. Rev. Lett. 81 (1998) 1562 doi:10.1103/PhysRevLett.81.1562 [hep-ex/9807003].

[3] Q. R. Ahmad et al. [SNO Collaboration], Phys. Rev. Lett. 89 (2002) 011301 doi:10.1103/PhysRevLett.89.011301 [nucl-ex/0204008].

[4] S. Abe et al. [KamLAND Collaboration], Phys. Rev. Lett. 100 (2008) 221803 doi:10.1103/PhysRevLett.100.221803 [arXiv:0801.4589 [hep-ex]].

[5] Ziro Maki, Masami Nakagawa, Shoichi Sakata; Remarks on the Unified Model of Elementary Particles, Progress of Theoretical Physics, Volume 28, Issue 5, 1 November 1962, Pages 870-880, https://doi.org/10.1143/PTP.28.870.

[6] D. A. Dwyer [Daya Bay Collaboration], Nucl. Phys. Proc. Suppl. 235-236 (2013) 30 doi:10.1016/j.nuclphysbps.2013.03.007 [arXiv:1303.3863 [hep-ex]].

[7] P. Adamson et al. [MINOS Collaboration], Phys. Rev. Lett. 110 (2013) no.25, 251801 doi:10.1103/PhysRevLett.110.251801 [arXiv:1304.6335 [hep-ex]].

[8] K. Abe et al. [T2K Collaboration], Phys. Rev. Lett. 112 (2014) no.18, 181801 doi:10.1103/PhysRevLett.112.181801 [arXiv:1403.1532 [hep-ex]].

[9] C. Patrignani et al. [Particle Data Group], Chin. Phys. C 40 (2016) no.10, 100001. doi:10.1088/1674-1137/40/10/100001

[10] S. Adrian-Martinez et al. [KM3Net Collaboration], J. Phys. G 43 (2016) no.8, 084001 doi:10.1088/0954-3899/43/8/084001 [arXiv:1601.07459 [astro-ph.IM]]. 
[11] R. Acciarri et al. [DUNE Collaboration], "Long-Baseline Neutrino Facility (LBNF) and Deep Underground Neutrino Experiment (DUNE) : Volume 2: The Physics Program for DUNE at LBNF," arXiv:1512.06148 [physics.ins-det].

[12] K. Abe et al. [Hyper-Kamiokande Working Group], "A Long Baseline Neutrino Oscillation Experiment Using J-PARC Neutrino Beam and Hyper-Kamiokande," arXiv:1412.4673 [physics.ins-det].

[13] G. Mention, M. Fechner, T. Lasserre, T. A. Mueller, D. Lhuillier, M. Cribier and A. Letourneau, Phys. Rev. D 83 (2011) 073006 doi:10.1103/PhysRevD.83.073006 [arXiv:1101.2755 [hep-ex]].

[14] W. Hampel et al. [GALLEX Collaboration], Phys. Lett. B 420 (1998) 114. doi:10.1016/S0370-2693(97)01562-1

[15] J. N. Abdurashitov et al. [SAGE Collaboration], Phys. Rev. C 59 (1999) 2246 doi:10.1103/PhysRevC.59.2246 [hep-ph/9803418].

[16] A. Aguilar-Arevalo et al. [LSND Collaboration], Phys. Rev. D 64 (2001) 112007 doi:10.1103/PhysRevD.64.112007 [hep-ex/0104049].

[17] A. A. Aguilar-Arevalo et al. [MiniBooNE Collaboration], Phys. Rev. Lett. 103 (2009) 111801 doi:10.1103/PhysRevLett.103.111801 [arXiv:0904.1958 [hep-ex]].

[18] A. A. Aguilar-Arevalo et al. [MiniBooNE Collaboration], Phys. Rev. Lett. 102 (2009) 101802 doi:10.1103/PhysRevLett.102.101802 [arXiv:0812.2243 [hep-ex]].

[19] A. A. Aguilar-Arevalo et al. [MiniBooNE Collaboration], Phys. Rev. Lett. 110 (2013) 161801 doi:10.1103/PhysRevLett.110.161801 [arXiv:1303.2588 [hep-ex]].

[20] P. Adamson et al. [MINOS Collaboration], Phys. Rev. D 81 (2010) 052004 doi:10.1103/PhysRevD.81.052004 [arXiv:1001.0336 [hep-ex]].

[21] G. Karagiorgi et al., Phys. Rev. D 80 (2009) 073001 Erratum: [Phys. Rev. D 81 (2010) 039902] doi:10.1103/PhysRevD.81.039902, 10.1103/PhysRevD.80.073001 [arXiv:0906.1997 [hep-ph]].

[22] J. Kopp et al., JHEP 1305 (2013) 050 doi:10.1007/JHEP05 (2013) 050 [arXiv:1303.3011 [hep-ph]].

[23] C. Giunti et al., Phys. Rev. D 88 (2013) 073008 doi:10.1103/PhysRevD.88.073008 [arXiv:1308.5288 [hep-ph]]. 
[24] M. Antonello et al. [MicroBooNE and LAr1-ND and ICARUS-WA104 Collaborations], "A Proposal for a Three Detector Short-Baseline Neutrino Oscillation Program in the Fermilab Booster Neutrino Beam," arXiv:1503.01520 [physics.ins-det].

[25] A. A. Aguilar-Arevalo et al. [MiniBooNE Collaboration], Phys. Rev. D 79 (2009) 072002 doi:10.1103/PhysRevD.79.072002 [arXiv:0806.1449 [hep-ex]].

[26] P. Adamson et al. [MINOS Collaboration], Phys. Rev. Lett. 117 (2016) no.15, 151803 doi:10.1103/PhysRevLett.117.151803 [arXiv:1607.01176 [hep-ex]].

[27] M. G. Aartsen et al. [IceCube Collaboration], Phys. Rev. Lett. 117 (2016) no.7, 071801 doi:10.1103/PhysRevLett.117.071801 [arXiv:1605.01990 [hep-ex]].

[28] C. Rubbia, "The Liquid Argon Time Projection Chamber: A New Concept for Neutrino Detectors," CERN-EP-INT-77-08 (1977).

[29] A. M. Szelc [LArIAT Collaboration], JINST $\mathbf{8} \quad$ (2013) C09011. doi:10.1088/1748-0221/8/09/C09011

[30] S. Amerio et al. [ICARUS Collaboration], Nucl. Instrum. Meth. A 527 (2004) 329. doi:10.1016/j.nima.2004.02.044.

[31] C. Anderson et al., JINST 7 (2012) P10019 doi:10.1088/1748-0221/7/10/P10019 [arXiv:1205.6747 [physics.ins-det]].

[32] V. Boccone, 2009 IEEE Nuclear Science Symposium Conference Record (NSS/MIC), Orlando, FL, (2009), pp. 1597-1600.

[33] S. Kubota, M. Hishida, M. Suzuki and J. z. Ruan(Gen, Phys. Rev. B 20 (1979) no.8, 3486. doi:10.1103/PhysRevB.20.3486

[34] H. Chen et al. [MicroBooNE Collaboration], "Proposal for a New Experiment Using the Booster and NuMI Neutrino Beamlines: MicroBooNE," FERMILAB-PROPOSAL$0974(2007)$.

[35] A. M. Szelc [SBND Collaboration], JINST 11 (2016) no.02, C02018. doi:10.1088/1748-0221/11/02/C02018

[36] R. Acciarri et al. [ArgoNeuT Collaboration], JINST 8 (2013) P08005 doi:10.1088/1748-0221/8/08/P08005 [arXiv:1306.1712 [physics.ins-det]]. 
[37] F. Cavanna et al. [LArIAT Collaboration], "LArIAT: Liquid Argon In A Testbeam," arXiv:1406.5560 [physics.ins-det].

[38] SBND building pictures, http://neutrino.uchicago.edu/blog/2017/06/. Accessed $22 / 09 / 2017$.

[39] C. Adams et al., "LAr1-ND: Testing Neutrino Anomalies with Multiple LAr TPC Detectors at Fermilab," FERMILAB-PROPOSAL-1053 (2013).

[40] M. Soderberg et al., "SBND APA Technical Design Report", SBND internal document N. $1531(2017)$.

[41] Fermilab Test Beam Facility (FTBF), http://www.ftbf.fnal.gov. Accessed $04 / 08 / 2017$.

[42] T. Akiri et al. [LBNE Collaboration], "The 2010 Interim Report of the Long-Baseline Neutrino Experiment Collaboration Physics Working Groups," arXiv:1110.6249 [hep$\mathrm{ex}]$.

[43] W. Foreman [LArIAT Collaboration], "First Run of the LArIAT Testbeam Experiment," arXiv:1511.00305 [physics.ins-det] (2015).

[44] I. Nutini [LArIAT Collaboration], J. Phys. Conf. Ser. 689 (2016) no.1, 012020. doi:10.1088/1742-6596/689/1/012020

[45] P. Kryczynski, JINST 11 (2016) no.02, C02086. doi:10.1088/1748-0221/11/02/C02086

[46] W. Foreman, "Single photoelectron rates update", SBND internal document N. 2199.

[47] R. Acciarri et al. [MicroBooNE Collaboration], JINST 12 (2017) no.08, P08003 doi:10.1088/1748-0221/12/08/P08003 [arXiv:1705.07341 [physics.ins-det]].

[48] R. Acciarri et al. [MicroBooNE Collaboration], JINST 12 (2017) no.03, T03003 doi:10.1088/1748-0221/12/03/T03003 [arXiv:1609.06169 [physics.ins-det]].

[49] T. Ohama, N. Ishihara, S. Takeda, H. Okuma and K. Konno, Nucl. Instrum. Meth. A 410 (1998) 175. doi:10.1016/S0168-9002(98)00224-1

[50] M. Soderberg et al., "APA Geometry Board Documentation for Electrical Review", SBND internal document N. 1351 (2016).

[51] A. Szelc, S. Tufanli [protoDUNE], "APA Commissioning Results", DUNE internal document N. 5576 (2017). 
[52] R. Acciarri et al. [DUNE Collaboration], "Long-Baseline Neutrino Facility (LBNF) and Deep Underground Neutrino Experiment (DUNE) : Volume 1: The LBNF and DUNE Projects," arXiv:1601.05471 [physics.ins-det].

[53] D. Garcia-Gamez et al., "Light System Simulation Studies in SBND", SBND internal document N. 1155 (publication in preparation) (2016).

[54] D. Garcia-Gamez, JINST $11 \quad$ (2016) no.01, $\quad$ C01080 doi:10.1088/1748-0221/11/01/C01080 [arXiv:1511.04611 [physics.ins-det]].

[55] VIKUITY Enhanced Specular Reflector Film (ESR), http://products3.3m. com/catalog/us/en001/electronics_mfg/vikuiti/node_7358GD8CMRbe/root_ GST1T4S9TCgv/vroot_S6Q2FD9X0Jge/gvel_B6D4SWL5QCgl/theme_us_vikuiti_3_0/ command_AbcPageHandler/output_html. Accessed 19/09/2017.

[56] DF2000 specular film, https://www.digikey.co.uk/product-detail/en/3m/ 75347099659/75347099659-ND/4988904. Accessed 19/09/2017.

[57] B. Rebel et al, (2011) J. Phys.: Conf. Ser. 308012023.

[58] G. Carugno, B. Dainese, F. Pietropaolo and F. Ptohos, Nucl. Instrum. Meth. A 292 (1990) 580. doi:10.1016/0168-9002(90)90176-7

[59] MTS test stand measurement of DF2000 foil, https://lartpc-docdb.fnal. gov/cgi-bin/private/RetrieveFile?docid=465\&filename=Material_Test_ Reflective_Film_all.pdf\&version=7. Accessed 13/09/2017.

[60] M. Cascella, "SBND High Voltage Feed-through and CPA Final Design Report", SBND internal document N. 1125 (2016).

[61] R. Acciarri et al., (2011) J. Phys.: Conf. Ser. 308012005.

[62] R. Francini et al., 2013 JINST 8 C09010.

[63] W. Foreman, LARTPC-doc-1938-v1 (2016).

[64] LArSoft, http://larsoft.org. Accessed 17/09/2017.

[65] G4beamline simulation program, http://www.muonsinternal.com/muons3/ G4beamline. Accessed 17/09/2017.

[66] Fermilab Hierarchical Configuration Language, https://cdcvs.fnal.gov/redmine/ projects/fhicl/wiki. Accessed 17/09/2017 
[67] CORSIKA simulation program, https://www.ikp.kit.edu/corsika/. Accessed $26 / 09 / 2017$. 